\title{
Medical narratives in the electronic medical record: towards a searching structure with optimal granularity
}

Citation for published version (APA):

Tange, H. J. (1997). Medical narratives in the electronic medical record : towards a searching structure with optimal granularity. [Doctoral Thesis, Maastricht University]. Universiteit Maastricht. https://doi.org/10.26481/dis.19970410ht

Document status and date:

Published: 01/01/1997

DOI:

10.26481/dis.19970410ht

Document Version:

Publisher's PDF, also known as Version of record

\section{Please check the document version of this publication:}

- A submitted manuscript is the version of the article upon submission and before peer-review. There can be important differences between the submitted version and the official published version of record.

People interested in the research are advised to contact the author for the final version of the publication, or visit the DOI to the publisher's website.

- The final author version and the galley proof are versions of the publication after peer review.

- The final published version features the final layout of the paper including the volume, issue and page numbers.

Link to publication

\footnotetext{
General rights rights.

- You may freely distribute the URL identifying the publication in the public portal. please follow below link for the End User Agreement:

www.umlib.nl/taverne-license

Take down policy

If you believe that this document breaches copyright please contact us at:

repository@maastrichtuniversity.nl

providing details and we will investigate your claim.
}

Copyright and moral rights for the publications made accessible in the public portal are retained by the authors and/or other copyright owners and it is a condition of accessing publications that users recognise and abide by the legal requirements associated with these

- Users may download and print one copy of any publication from the public portal for the purpose of private study or research.

- You may not further distribute the material or use it for any profit-making activity or commercial gain

If the publication is distributed under the terms of Article $25 \mathrm{fa}$ of the Dutch Copyright Act, indicated by the "Taverne" license above, 
Medical narratives in the electronic medical record Towards a searching structure with optimal granularity 
Dit proefschrift kwam tot stand in het kader wan het instituut ExTra, deel uitmakend van de landelijke onderzoeksschool CaRe (Netherlands School of Primary Care Research), in 1995 erkend door de KNAW.

Q H.J. Tange

Medical narratives in the electronic medical record - Towards a searching structure with optimal granularity / Huibert $\mathbf{J}$. Tange

Proefschrift Maastricht - Met literatuuropgave - Met Nederlandse samenvatting ISBN 90-9010429-1

Trefwoorden: electronic medical record / medical narratives / information retrieval

Onslag: Huibert Tange

Drukwerk: Datawyse | Universitaire Pers Maastricht 


\title{
Medical narratives in the electronic medical record
}

Towards a searching structure with optimal granularity

\author{
PROEFSCHRIFT
}

ter verkrijging van de graad van doctor aan de Universiteit Maastricht, op gezag van de Rector Magnificus, Prof. Mr. M.J. Cohen, volgens het besluit van het College der Decanen, in het openbaar te verdedigen op donderdag 10 april 1997 om 16:00 uur

door

\section{Huibert Johannes Tange}

geboren op 19 december 1958 te Dordrecht 


\section{Promotorem}

Prof.dr.ir. A. Hasman

Prof.dr. P.F. de Vries Robbé

\section{Co-promotor}

Dr. H.C. Schouten

\section{Beoordelingscommissie}

Prof.dr. H.F.P. Hillen

(voorzitter)

Prof.dr.ir. JiH. van Bemmel (Erasmus Universiteit Rotterdam)

Prof.dr. G.H. Blijham (Universiteit Utrecht)

Prof.dr. G. Kootstra

Prof.dr. J. Troost

Aan de drukkosten van dit proefschrift werd bijgedragen door: Care for Care b.v., Stichting DIV Automatisering in de Gezondheidszorg, Hewlett-Packard Nederland b.v., Jalema b.v., PIE Medical Benelux b.v., SMS Cendata b.v. en Stichting SAZZOG.

Met dank aan alle collega's, familieleden en vrienden die - elk op hun eigen wijze hebben bijgedragen aan de totstandkoming van dit proefschrift.

Mijn speciale dank gaat uit naar Jeroen Donkers en Vic Dreessen, voor hun bijdrage aan de ontwikkeling van het systeem, en aan Roy Lalisang, voor zijn hand- en spandiensten bij de voorbereiding van de proefopzet. 
Aan mijn ouders

Voor Birgitia, Karlijn en Koen 



\section{Contents}

Page

1. General introduction 9

2. Is the paper medical record really obsolete? 17

3. Medical narratives and electronic medical records -- A literature review 29

4. How to approach the structuring of the medical record? - Towards a multipleview searching structure

5. A conversation model of medical practice - How to get a process view on shared medical care

6. An experimental electronic medical-record system with multiple views on medical narratives

7. The gramularity of medical narratives and its effect on the speed and completeness of information retrieval

8. Appendix

9. Summary, conclusions, and discussion

10. Samenvatting

11. Curriculum Vitae 



\subsection{Introduction}

All medical-care activities involve gathering, analysing, or using medical data. Medical (or clinical) data comprise patient findings, medical decisions, and information about medical actions. ${ }^{2}$ The principle repository of medical data about a single patient is the medical record. Other terms used are clinical record, patient record, and health record. These distinct terms denote the same instrument covering information from different scopes. For example, a clinical record concerns hospital care rather than general practice ${ }^{3}$ and a patient record reflects the multi-disciplinary character of care. ${ }^{4}$ Nonetheless, these terms are often treated as synonyms. Recently, the Medical Record Institute (USA) proposed a distinction between medical records used within a single health-care institute, patient records used across health-care enterprises, and health records which also take the patient's well-being and other health-related information into account. ${ }^{5}$ Object of study in this thesis is the inpatient medical record.

\subsection{Functions of the medical record}

The primary function of the medical record is to provide the physician with information needed for direct medical practice." Traditionally, the medical record served as an aide memoir for the individual physician. The last decades showed an explosive growth of medical knowledge and technology, increasing the complexity of health care. This growing complexity had several consequences: a further specialisation of physicians, a trend to multi-disciplinary care with a growing need for physicians and other professionals to co-operate and communicate, and the production of a an ever growing mass of data concerning the patients' diseases and treatments. More and more, the medical record has become a multi-authored communication platform for professionals of different disciplines sharing responsibility for the care of an individual patient. But the primary purpose of the medical record remained the same: to provide the physician with information for direct medical practice. In fact, its traditional use as an aide memoir can be understood as a special kind of communication, in which sender and receiver do not differ in person but only in time.

Other, more or less traditional, applications of the medical record are to serve as evidence for reimbursement and litigation, and as a source of information for research and medical education. ${ }^{1, \$}$ Recent governmental pressure towards a more efficient and effective health-care system resulted in a new application of the medical record. This pressure, namely, induced a growing interest of hospital management and healti-care

\footnotetext{
a Although traditionally a masculine profession, the proportion of women under physicians is rapidly growing. In the Netherlands, the number of female already exceeds the number of male medical students. Yet, if only to identify myself better with the user of the medical record, I will use masculine pronouns when referring to physicians. Of course, their fernalle equivalents can be read instead.
} 
insurance compantes in details about the cost and quality of patient care. These parties wish to use the medical record as an information source for management and control of paticnt cate.

\subsection{Motivation for this thesis}

As a result of this growing, diverse, need for medical data, the gap between the role the medical record can play and the role it should play is widening. It is generally agreed upon, that paper-based medical records cannot support the above mentioned functions properly. "From electronic medical records, instead, many advantages are expected: Yet, despite more than twenty-five years of practical experience in pioneering environments, the development and dissemination of electronic medical-record systems has been slow. Various reasons have been suggested for this lack of progress: not only technical arguments but also other ones, such as the wnruliness of narrative data, a defensive attitude of physicians towards the use of computers, and unsolved problems with respect to privacy.

To remove these barriers, it was suggested that the time has come to replace the technology push by a user pull. "The question is, however, which users will provide the strongest pull. At the moment, the parties that aim at a more effective and efficient health care are the biggest proponent of (some form of) electronic medical records. Their interest is to manage, control, and formalise the work of physicians and other professionals. "Their user pull might lead to a kind of electronic medical-record systems that will be moulded by the bureaucratic needs of the organisation rather than by the interest of direct medical practice. As a consequence, physicians might become subordinate to bureaucratic control with the danger of having their own medical record turned into a Trojan Horse."

On the other hand, if physicians would take the initiative for computerising the medical record, it would be up to them to take advantage of the facilltating and coordinating potential of information technology. If physicians generate the strongest user pull, the medical record will be reinforced in its primary function and, once in electronic format, become the kemel of a medical workstation that provides the physician with information and decision support for direct medical practice. As a result, the physician will remain fully responsible for and creative in finding bestfitting solutions for the medical problems they encounter.

But before physicians will be able to pull, they need a clear prospect of what an electronic medical record could mean for them. I believe, that the next few years will be crucial. With this thesis, 1 hope to contribute to this prospect.

\subsection{Goal of this thesis}

Important information for direct medical practice is documented in medical history, physical examination, and progress notes. Electronic medical-record systems that do not include these so-called narrative parts will never become superior to paper-based medical records. However, these parts have been characterised as unruly, in the sense 
that they are difficult to process by the computer. Problems have to be solved with respect to the entry, storage, and retrieval of medical narratives, before an electronic medical record can be considered as a real altemative for paper charts. Many studies are conducted with respect to the first two issues (see Chapter 3 ).

This thesis focuses on the third issue. Aim of this thesis is to explore how information technology can best be used to facilitate, for direct medical practice, the retrieval of information from the medical record. In particular, it is investigated how, in an electronic medical-record system, searching information from medical narratives can be facilitated by optimising their paragraph structure.

\subsection{Definitions}

The Medical Record Institute (USA) proposed five different terms for patient information systems that reflect different levels of computerisation (levels 1-3) and information of different scopes (levels $3-5$ ). ${ }^{5}$ The different scopes were already mentioned in Paragraph 1.1. The different levels of computerisation are: automated (partly paper-based, partly computer printouts), computerised (entered on paper forms and scanned into the computer without character recognition), and electronic or computer-based (direct computer input and integration with other sources). According to these definitions, an electronic medical record is the repository of medical data within one health-care enterprise that is characterised by direct data input and integration of different sources.

In this thesis, I focus on medical narratives. Medical narratives are clinical data that are traditionally recorded as a natural-prose story. Usually this is the case with qualitative, physician-gathered, medical data such as medical history, physical examination, and progress notes. But also reports of special procedures (radiology, pathology, endoscopy) are usually presented as natural prose, as is also the correspondence between specialist and general practitioner. In this thesis, I restrict the use of the term medical narratives to the sections medical history (in particular present illness and systems review), physical examination, and progress notes.

In this thesis, I investigate the structure of medical narratives with the aim to facilitate the retrieval of information. I consider information retrieval from the medical record as a two-step process. First, the user has to navigate through the medical record to locate sections or paragraphs that may contain relevant information. In this phase, the user is guided by the structure of the medical record, which I define as the organisation of medical data in sections and paragraphs as well as in relation to one another. Second, the user has to read the content of the selected paragraphs to retrieve the information needed. In this phase, the format of medical data, is important. The problem of formatting medical narratives concerns the understandability of their content and amounts to making a choice between storing them as free text or as controlled expressions. Controlled expressions are derived from a controlled vocabulary or medical terminology. At present, standardisation of medical terminology is an issue engaging many standardisation bodies all over the world, ${ }^{10.11}$ and medical narratives in electronic medical records are increasingly stored as 
controllad expressions. The usual format for presentotion, however, remains free text. Since I testrict myself to investigating the retrieval of information, I have chosen the usual presentation format and do not go into the subject of the storage format of medical narratives.

\subsection{Specific research questions and composition of this thesis}

I try to attain the goal of this thesis by successively studying the following research questions:"

- How adequate does the current paper medical record perform its various purposes?

In 1991, the Committee on the Patient Record (USA) concluded that the paper medical recond has become obsolete. I tried to verify these conclusions in a Dutch university hospital with the aim also to elucidate some requirements for an electronic medicalrecord system. For this reason, I carried out a questionnaire-based survey among eighty-five hospital physicians. This study is reported in Chapter 2.

- Which research has been performed with respect to the inclusion of medical narratives in electronic medical-record systems, and which measures have been proposed to facilitate the retrieval of information from medical narratives for direct medical practice?

To answer this question, I carried out a literature survey. From this survey, which is reported in Chapter 3, I conclude that there is no consensus about how to structure medical narratives for searching purposes and that the process of retrieving information from medical narratives is a relatively unexplored research domain.

The remainder of this thesis is devoted to the question how medical narratives must be structured to optimally support the physician when searching in formation for direct medical practice.

- Which perspectives on medical narratives are helpful for searching information from medical narratives and how can these views be implemented in an electronic medical-record system?

To support the entry, storage, and retrieval of patient medical data, electronic medicalrecond systems can use the principles of the source-oriented, time-oriented, and problem-oriented medical record. In Chapter 4, I translate these principles into four possible views on medical data: source view, problem view, time view, and process view. I describe how these views can be combined in the conceptual data model of an electronic medical-record system and demonstrate that this data model is also applicable for medical narratives.

- How can the medical record best reflect the process of medical care and how can this process view be implemented in an electronic medical-record system?

The process view is difficult to specify. In Chapter 5, I report how an appropriate process view can be inferred by combining three current approaches to systematic medical practice: Weed's problem-driven model, Elstein's diagnosis-directed model, and Rector's model of the faithful medical record. In doing so, I bring these models 
together in one conversation model of medical practice. I also demonstrate how from this conversation model a process view can be deduced that can be part of the conceptual data model of an electronic medical-record system.

Based on the principles described in Chapters 4 and 5, we developed a prototype electronic medical-record system specifically designed (1) to facilitate the retrieval of information from medical narratives and (2) to support research related to the use of the medical record. In Chapter 6, I describe the design and architecture of this system and report its use for a specific study.

- How should medical narratives be partitioned to support the physician effectively and efficiently when retrieving information from the medical record for direct medical practice?

The added value of an electronic medical-record system that provides a searching structure with different views on medical narratives depends on the granularity of the medical narratives. I conducted an experiment to investigate which granularity facilitates the retrieval of information from medical narratives for direct medical practice best, in terms of search speed and completeness of retrieved information. In Chapter 7 , I report this study, in which thirty-six hospital physicians were involved.

\subsection{References}

1. Shortliffe EH, Barnett GO. Medical data: their acquisition, storage, and use. In: Shortliffe EH, Perreault LE, eds. Medical Informatics - Computer Applications in Health Care. Reading MA: Addison-Wesley, 1990:37-69.

2. Wyatt JC. Clinical data systems, Part 1: Data and medical records. Lancet $1994 ; 344: 1543-7$.

3. Reiser SJ. The clinical record in medicine. Part 2: Reforming content and purpose. Ann Intern Med 1991; 114:980-5.

4. Dick RS, Stein EB. The computer-based patient record - an essential technology for health care. Washington DC: National Academy Press, 1991.

5. What is an Electronic Patient Record? World Wide Web: Medical Records Institute, $1995:$ htpm://ww medrecinst.com/mri/levells. html.

6. Van Londen J. Dutch health care - a study in purple. Lancet 1996, 347:1220 1231 .

7. McDonald $\mathrm{CJ}$, Tierney WM. Computer-stored medical records. Their future role in medical practice. Jama 1988; 259:3433-40.

8. Telematics Applications Programme (1994-1998): Workprogramme. Brussels: European Commission DG XIII, 1994:annex: 89-98.

9. Lamberts $H_{3}$ Hofmans-Okkes 1 . The generic patient record: an alliance between patient documentation and medical informatics. Methods Inf Med 1996:35:5-7.

10. Standards for medical identifiers, codes, and messages needed to create an efficient computerstored medical record. American Medical Informatics Association. J Am Med Inform Assoc $1994 ; 1: 1-7$.

11. Hammond WE. The role of standards in creating a health information infrastructure. Ind $J$ Biomed Comput 1994; 34:29-44. 



\title{
2. Is the paper medical record really obsolete?
}

\author{
H.J. Tange
}

Department of Medical Informatics, Maastricht University

In 1991, the Committee on Improving the Patient Record, appointed by the Institute of Medicine (USA), concluded that the paper medical record had become obsolete and that a nation-wide computerisation of the medical record should be promoted. The Committee's report had a large impact on the discussion about medical-record keeping, also outside the USA. Here, we report about a study that investigates whether the Committee's conclusions about the paper medical record are shared by hospital physicians in a Dutch university hospital. We conducted a questionnaire-based survey among 85 pllysicians from 15 clinical departments to assess their opinion about the quality of the paper medical record. The interviewees were asked to judge the quality of the paper medical record with respect to various aspects of general use, data entry, and information retriewal. It was concluded, that the participating clinicians were far more positive about the quality of the paper medical record than could be expected from the Committee's report. Possible explanations for this discrepancy are discussed, like nationbound differences in medical culture, a possible bias caused by the teaching character of the hospital, and the difference between evaluating the medical record as such on one side and its use on the other. Main conclusion of our study is, that one should be careful when using the Committee"s report as a motivation for replacing the paper medical record.

\section{Published as:}

Tange HJ. The paper-based medical record - Is it really obsolete? Comput Methods Programs Biomed 1995; 48:127-131.

\section{Extended version}


Chapren 2 


\subsection{Introduction}

Clinical care has a multi-disciplinary character, which necessitates proper communication between professionals. The medical record is the most important touchable reflection of this communication. It is used by professionals of various disciplines and other parties, each with its own special interest. Hence, terms like patient record and health record are used as alternatives for medical record.' It is generally recognised, that the medical record in its present paper format is not able to satisfy the diversity of current and future information needs. From computer-based medical record keeping, on the other hand, many advantages are expected. ${ }^{2}$ Both in the USA and in Europe, concerted actions are initiated to facilitate the overall dissemination and implementation of computer-based medical records. ${ }^{1.3}$

In 1989, the Institute of Medicine (USA) established the Committee on Improving the Patient Record with the task to determine the impediments to the dissemination of computer-based medical records and to propose a national strategy to overcome these impediments. Part of this study concerned a literature survey to examine the quality of the paper medical record. For more details about this survey we refer to the Committee's report." The Committee did not encounter substantial evidence of strengths of paper medical record keeping in the literature. Instead, some positive aspects were formulated based on the members' own experience (Table 2-1). Weaknesses of the paper medical record, on the other hand, were found in many publications. The Committee concluded that the paper medical record is an invalid instrument for its purpose, and suggested to promote the implementation of computerbased medical records all over the USA within 10 years.

From its publication in 1991, the Committee"s report has been a standard reference for future policy, research, and development with respect to the medical record, both inside and outside the USA. For example, in the Yearbook of Medical Informatics of 1994, a selection of journal articles about medical-informatics subjects published the

Table 2-1:

Qualifications of the paper medical record (as determined by the Committee on Improving the Patient Record)

Strengths of paper medical records

- They are familiar to the user

- They are easy to carny to the point of care, e.g., the bedside of the patient

- They are easy to browse

- They allow the user all freedom in choosing which data items to record, in which words, and where in the medical record

Weaknesses of paper medical records

- Information retrieval is ineffective and time-consuming

- Data are often missing, illegible, or inaccurate

- They can only be used at one location at a time

- An integrated patient record cannot be achieved 
year before, all five articles in the section "computer-based patient records" refer to this book. ${ }^{4}$ For us, this acceptance gave rise to the question whether the Committee's conclusions are shared by the daily users of the paper medical record. Practitioners build on a century old tradition of paper medical record keeping, ${ }^{5}$ so it can be imagined that they are hesitant to set aside this tradition in favour of the new and unknown.

\subsection{Objective, material and method}

In this chapter, we present the results of a study that had as goal to examine to which extent the Committee's assessment of the quality of the paper medical record is supported at the level of local practice in - for example - a Dutch hospital. The study was carried out in 1993. We developed a paper questionnaire with fifty-five statements about the quality of the paper medical record, for the purpose to study the appreciation of the paper medical record and the need for improvement.

In this questionnaire, the participants had to assess a list of quality aspects with respect to the paper medical record, grouped into three main categories: general use, data entry, and information retrieval. For each aspect, they had to score their satisfaction with the paper medical record. To blind the participants for the Committee's conclusions, we formulated the statements in such a way that agreement always implied a positive appreciation. For example, the Committee's finding "data are often difficult to retrieve" was reformulated as "data are easy to retrieve" (do you agree?). The level of agreement had to be expressed on an five-points ordinal scale (Likert). We also asked the participants to judge the relative impartance of each quality aspect. ${ }^{6}$ A positive quality judgement (score $4-5$ ) together with a high importance was interpreted as a wish to maintain the status quo, while a negative or neutral quality judgement (score 1-3) together with a high importance was interpreted as a need for improvement. Additionally, we inserted some questions about the frequency of and reasons for using the medical record, and also some questions to obtain some characteristics of the participating physicians. The questionnaire was self explaining and its completion took between 20 and 30 minutes.

To maximise the response, it was decided not to approach potential participants directly after simple randomised sampling, but to use a procedure of stratified purposive sampling. We asked the heads of all clinical departments to distribute the questionnaire among a part of their personnel (one third was given as indication) that they considered representative for the groups of specialists and residents. A consequence of this sampling procedure was, however, that the exact response rate could not be determined.

We planned to test the influence of the level of expertise and the type of discipline on the users" judgement, for which reason we grouped the disciplines into four categories: surgical specialisms, non-surgical specialisms, psychiatry, and supporting clinical specialisms (Table 2-2). We carried out a non-parametric analysis of variance (Kruskal-Wallis) to test the overall agreement between the four categories (required level of significance $\mathrm{p}<0.01$ ). In case of significance, we subsequently calculated the pair-wise contrasts between the different categories (Mann-Whitney) to determine 
significant differences between individual categories. For these pair-wise contrasts we had to correct the required level of significance for the number of pairs (six) according to Bonferroni (required level of significance $p<0.0017$ ). ${ }^{\text {? }}$

The clinicians ${ }^{*}$ assessment must be interpreted in the context of their actual way of information processing. The clinicians under study used paper medical records. During hospitalisation of the patient, the outpatient record was included in the inpatient record, while the nursing record was kept separately. During outpatient care, only the outpatient record was available, and information about inpatient episodes had to be extracted from discharge letters. ${ }^{8}$ Most results of auxiliary services (e.g. laboratory results) were stored in the BAZIS hospital information system. ${ }^{9}$ A print-out of these results was included in the paper medical record. At the time of the study, direct interaction of physicians with the hospital information system was infrequent.

\subsection{Results}

Response (Table 2-2) - From the 22 clinical departments that were approached, 15 $(68 \%)$ finally took part in the survey. Reasons for non-participation were high workload $(4 x)$, unconditional non co-operation with questionnaires $(2 x)$, and nonresponse after initial promise to co-operate (1x). A total of 85 questionnaires was returned, which is $26 \%$ of the population under study (29\% of the specialists and $23 \%$ of the residents).

\section{Usage of the medical record} (Table 2-3) - A majority of the participants used inpatient as well as outpatient medical records daily. Supporting clinical specialists used the outpatient record less than others (MannWhitney test, $\mathrm{p}<0.0017)$. The nursing record was consulted rarely. Most participants appreciated the present situation in which inpatient and outpatient record, and also medical and nursing record, are separated. Most participants, except supporting clinical specialists (Mann-Whitney test, $p<0.0017$ ), used the medical record daily as an aide memoir. Also for planning and communication purposes, the medical record was used daily. All these purposes were considered important. Less than

Table 2-2:

Number of participants per discipline

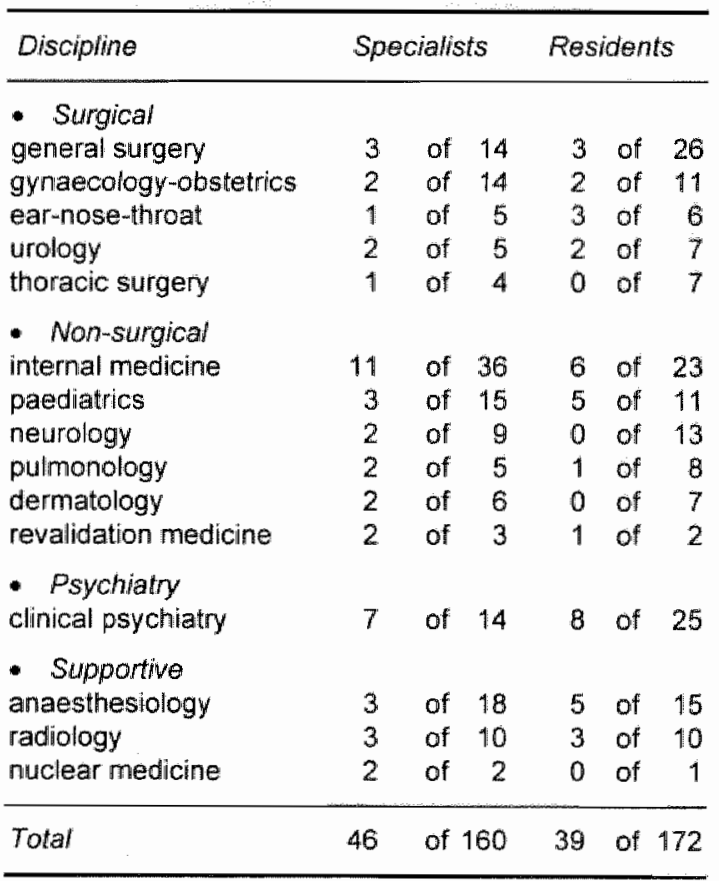


Table $2-3$.

Frequency of use and consictered importance (in percentages, $n=85$ )

\begin{tabular}{lcccc}
\hline & \multicolumn{1}{c}{ Frequency of use (\%) } & & \multirow{2}{*}{ Impontant (\%) } \\
\cline { 2 - 4 } & less than weekly & weakly & daily \\
\hline aide memoir & 12 & 20 & 68 & 85 \\
communication toot & 12 & 38 & 50 & 81 \\
planning of activities & 19 & 25 & 56 & 76 \\
adininistration & 68 & 15 & 17 & 37 \\
quality accounting & 37 & 33 & 30 & 68 \\
patient case discussion & 44 & 39 & 17 & 73 \\
research & 80 & 16 & 5 & 63 \\
meidical education & 86 & 11 & 4 & 38 \\
\hline
\end{tabular}

daily, but still regularly, the medical record was used for quality accounting and as a source for patient-case discussions. These purposes were also considered relevant. A majority of the participants considered the role of medical records as a source for research important, although most of them used the medical record less than once a week for this purpose. The other functions of the medical records (education, administration) were used infrequently and were also considered less important.

General quality aspects (Table $2-4$, upper part) - Most participants considered the paper medical record easy to carry to the bedside, but the satisfaction scores with respect to the availability at any location were diverging. Both aspects, however, were not considered important. The availability of the medical record at any time was judged satisfactory and worthwhile to be maintained. Supporting physicians scored lower on this aspect, though not significantly. Most participants considered the medical record complete enough with respect to the documentation of diagnostic and therapeutic interventions, but not with respect to the registration of decisions, outstanding orders, and planned activities. This incompleteness should be corrected. The information contained in the paper medical record was considered reliable and up to date enough by a comfortable majority of the participants. Most participants indicated, that the medical record was not sufficiently protected against violation of privacy and that this feature should be improved. Also, most participants had the experience that complete records or individual documents are at risk of getting lost, a situation which should also be avoided.

Quality of data entry (Table 2-4, middle part) - A vast majority was satisfied with the ease and speed of datit entry in the paper medical record. Also the freedom to choose which data to write down, at which level of detail, in which words, and where in the record was appreciated by most participants. All these aspects of freedom of expression, except the last one, were considered important enough to be maintained.

Quality of information retrieval (Table 2-1, lower part) - More than three quarters of the participants considered information entered by themselves easy to retrieve and well legible, in contrast with information entered by others. A small majority was satisfied with the speed of getting a first impression of the present state of a patient and wanted to keep it like this. However, only a minority was satisfied with 
the speed of getting full overview of the progress of treatment, and also with the speed of getting a first impression of both present state and progress of treatment. All these aspects should be improved. A majority of the participants was not satisfied with the speed with which specific data can be retrieved from the paper medical record, especially when looking for data that may or may not be present. This aspect should also be improved. With respect to the question whether information retrieval was impeded by a mass of irrelevant details, the opinions varied. Yet there was a small majority in favour of improving this aspect, because many of the neutrally scoring participants considered this an important feature. Overlooking relevant information was not considered a problem and neither an important issue by the majority.

\subsection{Discussion}

The most obvious result from our study is that, in contrast with the findings of the Committee on Improving the Patient Record,' the participating clinicians were rather satisfied with the paper medical record. They acknowledged the strong aspects that were formulated by the Committee, but agreed far less with the weaknesses that were attributed to the paper medical record (Table 2-4). They agreed with the Committee on the aspect that paper medical records are easy to carry to the bedside (however they did not consider it important), and that they offer enough freedom of expression. The ease of browsing through the medical record was only acknowledged when a quick impression of the present state of a patient had to be obtained, but the medical record was not considered good enough to obtain a quick impression of the progress of treatment. The participants did not agree with the fact that data, in general, were often incomplete, because the most important data, namely the results of interventions, were considered complete enough. The documentation of decisions, orders and plans was (although not explicitly considered unsatisfactory) judged important enough to be improved.

The critique is generally heard that data in the paper medical record are often unreliable. However, our participants disagreed with this firmly. Data in the paper medical record were judged correct and timely enough. Also in contrast with the literature, the absence of integration of the different paper patient records was not considered a problem. Some other opinions of the participants were in agreement with the literature. Searching for specific information was considered difficult and time consuming. Also the problem of illegible data was recognised, but only as far as it concemed data entered by others.

This discrepancy with the literature may have different causes. In the questionnaire, all aspects were presented as positive statements. This might have induced the participants to a more positive scoring behaviour then in the case of a mixture of positive and negative statements. On the other hand, formulating our statements according to the Committee"s conclusions would have been also triggering. Only a random mixture of positive and negative statements would be free of cues, but would probably lead to confusion when both satisfaction and importance had to be scored. 
Table 2-4:

Quality aspects of the paper medical record (in percentages, $n=85$ ).

- satisfaction level: - (unsatisfactory), 0 (neutral) + (satisfactory)

- agrae with Conmittee agree, disagree

- need for change: maintain, improve

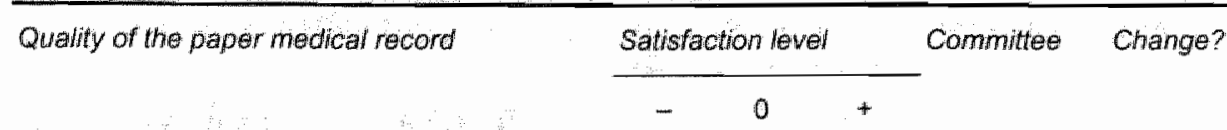

- General aspects

ease of carrying to the bedside availability on any time availlability on any locration completeness of...

... diagnostic intervertions

...therapeutic interventions

... decisions made

...orders made

... plans made

reliability of data

timeliness of data

privacy protection

risk of loss of..

... medical records

... medical data

$\begin{array}{cccll}12 & 9 & 79 & \text { agree } & \\ 29 & 2 & 68 & \text { agree } & \text { maintain } \\ 48 & 14 & 38 & & \\ & & & & \\ 24 & 12 & 65 & \text { disagree } & \text { maintain } \\ 24 & 19 & 58 & \text { disagree } & \text { maintain } \\ 28 & 29 & 42 & \text { agree } & \text { improve } \\ 32 & 25 & 44 & \text { agree } & \text { improve } \\ 32 & 34 & 34 & \text { agree } & \text { improve } \\ 8 & 19 & 73 & \text { disagree } & \text { maintain } \\ 9 & 16 & 74 & \text { disagree } & \text { maintain } \\ 60 & 19 & 21 & \text { agree } & \text { improve } \\ & & & & \\ 52 & 21 & 27 & \text { agree } & \text { improve } \\ 42 & 28 & 29 & & \text { improve }\end{array}$

- Data entry qualities

ease of data entry

speed of data entry

flexibility in recording.

... Which data to register

...in which level of detail

...in which words

...in which place

$\begin{array}{ccccc}11 & 12 & 78 & & \text { maintain } \\ 13 & 13 & 74 & & \text { maintain } \\ 5 & 16 & 79 & \text { agree } & \text { maintain } \\ 4 & 19 & 78 & \text { agree } & \text { maintain } \\ 9 & 11 & 80 & \text { agree } & \text { maintain } \\ 24 & 16 & 60 & \text { agree } & \end{array}$

- Information retrieval qualities

ease of information retrieval...

... retrieving own data

... retrieving other's data

legibility of data from...

... own handwriting

... other's handwriting

speed of getting an overview of...

... the progress of treatment: first impression

... the progress of treatment: full impression

...the present state: first impression

.. the present state: full impression

speed of finding specific data

speed of determining absent data

impediment of information retrieval by...

\begin{tabular}{|c|c|c|c|c|}
\hline 9 & 14 & 76 & disagree & maintain \\
\hline 48 & 18 & 34. & agree & improve \\
\hline 4 & 5 & 92 & disagree & maintain \\
\hline 59 & 20 & 21 & agree & improve \\
\hline 38 & 21 & 41 & disagree & improve \\
\hline 45 & 22 & 33 & agree & improve \\
\hline 311 & 18 & 52 & agree & maintain \\
\hline 40 & 27 & 33 & & improve \\
\hline 54 & 16 & 29 & agree & improve \\
\hline 71 & 15 & 14 & agree & improve \\
\hline 39 & 24 & 38 & & improve \\
\hline 25 & 38 & 38 & & \\
\hline
\end{tabular}


The hospital under study is a teaching hospital, which implies more interaction between clinicians than in general hospitals. This circumstance necessitates a more complete and accurate way of medical record keeping, which may contribute to a more positive appraisal of the paper medical record than can be expected in general.

The discrepancy we found may also be explained by different governmental and legal demands. In the USA, medical records are used as a legal document to account for one's acts. For example, in US legislation, acts that are not registered are accounted as if they had not taken place. ${ }^{10}$ Such an incentive for being complete and accurate is absent in The Netherlands (yet).

Also the difference in aspect studied may contribute to this discrepancy. The survey of the Committee refers to studies in which the paper medical record is evaluated as such,' while our own study focuses on the use of the paper medical record. Both these approaches are complementary, so both are important. In terms of technology assessment: we did not evaluate the medical record as part of the structure of health care, but as part of the process of health care. "In daily practice, clinicians use additional information sources, e.g. their own memory and informal contacts with colleagues, so shortcomings of the paper medical record can be compensated easily, even without notice of the user.

Another noteworthy element of our study was the large number of neutral satisfaction scores. This can be an indication of indifference about the quality of the medical record. If it is true that many physicians have a non-critical attitude towards the medical record, the quality of care is at risk. Since, the medical record is as much a medical device as medical instruments and drugs are, and the latter have to meet high quality standards before they are permitted to the market. In the USA, various types of clinical-information systems, such as laboratory systems, have to meet high FDAquality standards, ${ }^{12}$ so why not also subject the medical records (whether computerised or not) to legal standards of quality?

We end this discussion with some remarks about the validity of our study. When gathering test persons we faced the dilemma between a possible selection bias caused by a low response and a possible selection bias caused by the use of another more effective, procedure than randomised sampling. We judged the first risk more serious than the second, because the questionnaire concerned a non-clinical subject. This was the reason why we have chosen for stratified purposive sampling by the heads of the departments, delegating the responsibility for a representative sample to them. Given our aim to get participation of one third of the clinicians, the achieved participation rate of $26 \%$ of the total population under study is fairly acceptable. However, this sampling procedure did not give us information about possible selection bias on the level of individual physicians.

The external wallidity of our study is restricted by fact that only one hospital was involved. Another, more recent, study in The Netherlands involved a mixture of fortynine physicians from both academic and general hospitals. This study showed similar results in the sense that physicians were rather satisfied with the existing paper medical record and that most benefit of automation was expected in the presentation of results. ${ }^{13}$ Yet, it was not the intention of our case study to generalise the results to other 
hospitals, but only to verify the Committee's conclusions about the paper medical record in a specific site. Nevertheless, our results can be considered illustrative for the fact that one should be careful with projecting the Committee's conclusions to one's own situation.

\subsection{Conclusion}

The suggestion that the paper medical record has become obsolete in the eyes of physicians, was not confirmed in this study. What we learned is to be careful with projecting conclusions about medical record keeping, obtained from the literature, to a local situation. A negative opinion about paper medical records from the literature (often accompanied by promising descriptions of computer-based systems) should not be the only argument on which the decision to replace the paper medical record with a computer-based record should be based. Not only the features that need to be improved should be considered, but also the qualities of the paper medical record that the users want to preserve.

The user appreciation may be influenced by national differences, but also by sitespecific variables, such as the status of teaching hospital. Hence, an assessment of the users' appreciation of the paper medical record should be an essential argument when deciding whether or not to computerise the medical record. As long as physicians are satisfied with the paper medical record, it is not likely that there will be much enthusiasm for a change. The computer-based medical record is not yet a fully developed tool, and much research is still in progress. Nevertheless, it is time for the ordinary practitioner to follow these developments closely and - better - to participate in it, because only a broad clinical involvement can support the maturation of the electronic medical record.

\subsection{References}

1. Dick $\mathbb{R S} S_{3}$ Stein EB. The computer-based patient record - an essential technology for health care. Washington DC: National Academy Press, 1991.

2. McDonald $\mathrm{CJ}$, Tienney WM. Computer-stored medical records. Their future role in medical practice Jama 1988:259:3433-40.

3. Telematics Applications Programme (1994-1998): Workprogranme. Brussels: European Commission DG XIII, 1994:annex: 89-98.

4. van Benmel $\mathrm{JH}_{3}$ McCray AT. Advanced Communications in Health Care. Yearbook of Medical Informatics. Stutgart: Schattauer, 1994.

5. Reiser SJ. The clinical record in medicine. Part 2: Reforming content and purpose. Ann Intern Med 1991; 114:980-5.

6. Bailey J, Pierson S. Development of a tool for measuring and analyzing computer user satisfaction. Management Science 1983; 29:530-545.

7. Altman DG. Practical statistics for medical research. London: Chapman and Hall, 1991:611.

8. Tange HJ, Smeets RP. Information exchange between physicians and nurses. Comput Methods Programs Biomed 1994; 43:261-7.

9. Bakker AR. An integrated hospital information system in The Netherlands. MD Comput 1990; 7:91-97. 
10. Shortliffe EH, Barneti GO. Medical data: their acquisition, storage, and use In: Shortliffe EH. Perreault $L E$, eds. Medical Informatics - Computer Applications in Health Care. Reading MA: Addison-Wesley, 1990:37.69.

11. Donabedian A. The quality of care - How can it be assessed? I Am Med Assoc 1988;2160:17431748 :

12. Gamerman GE. FDA regulation of biomedical software. Proc Anmu Symp Comput Appl Med Care 1992:745-749.

13. Berger $\$$, Hilderink HGM. The medical doctor and the clinical information system: a feasibility study. Proc Amice 1995:15-23. 


\title{
3. Medical narratives and electronic medical records - A literature review
}

\author{
H.J. Tange ${ }^{a}$, A. Hasman", P.F. de Vries Robbéb, H.C. Schoutene \\ ${ }^{a}$ Department of Medical Informatics, Maastricht University \\ ${ }^{\text {b }}$ Department of Medical Informatics, Epidemiology, and Statistics, Nijmegen \\ University \\ ¿Department of Internal Medicine, Maastricht University Hospital
}

In this chapter we describe the state of the art and directions of current development and research with respect to the inclusion of medical narratives in electronic medical-recordit systems. We used information about twenty electronic medicallrecord systems as presented in the literature. We divided these systems into classical systems that matured before 1990 and now used in a broad range of medical domains, and experimental systems, more recently developed and, in general, more innovative. In the literature, three major challenges were addressed: facilitation of direct data entry, achieving unambiguous understandability of data, and facilitation of information retrieval. Promising approaches to tackle the first and second challenge are the use of dynamic data-entry forms that anticipate sensible input, and free-text data entry followed by natural-language interpretation. Both these approaches require a highly expressive medical terminology.

How to facilitate the retrieval of information from medical narratives has not much been studied. We found examples of facilitating the retrieval of information from medical narratives by presenting them as fluent prose, by optimising the screen design with fixed position cues, and by giving them a structure of indexable paragraphs that can be used in flowsheets. We conclude that further study is needed to develop an optimal searching structure for medical narratives.

Submitted for publication 


\subsection{Introduction}

The development and use of computer-based medical-record systems could be a major contribution to the improvement of health care. However, despite more than 30 years of exploratory work, medical records are still predominantly paper-based. In 1991 the Committee on Improving the Patient Record of the Institute of Medicine (USA) concluded, that no current system was capable of supporting the complete computerbased patient record, and suggested warious reasons why the development and diffusion of computer-based medical reconds were impeded.' These barriers were both technological and non-technological. As technological barriers were mentioned the immaturity of hand-held terminals, voice-recognition techniques, and intuitive humancomputer interfaces. As non-technological barriers were mentioned a lack of awareness of the capabilities and benefits of electronic medical records anong potential users and vendors, the absence of a technical infrastructure, and unsolved problems related to the protection of privacy. These barriers have in common that they mainly pertain to the narrative parts of the medical record. No wonder, that since the very beginning of computer-based medical records, ${ }^{2}$ developers have struggled with the capture of medical narratives.

In this literature review we try to describe the state of the art with respect to the computerisation of medical narratives and to elucidate the direction of current developments. We distinguish three aspects of the capture of medical nartatives: data entry, data storage, and information retrieval. In particular we are interested in literature concerning methods to facilitate the retrieval of information from medical narratives. We realise that many recent developments of electronic medical-record systems, especially by commercial vendors, have not (yet) resulted in scientific publications. Since it is difficult to get reliable information about unpublished developments, we have limited ourselves in this survey to peer-reviewed publications and books.

We use the term electronic medical record (EMR) - or its equivalent compurerbased medical record - according to the definition given by the Patient Recosd Institute (USA): "a repository for patient information within one health-care enterprise (e.g., within one hospital, HJT) that is supported by direct computer input and integrated with other information sources". "We consider this the best term to describe the intention of current systems. For reasons of convenience we shall use this term for all systems reviewed in this survey. As medical narratives we consider all qualitative (and semi-quantitative) data gathered by the physician. This term comprises the physician-gathered core parts of the medical record (medical history, physical examination, progress notes), and furthermore the reports of special procedures (radiology, pathollogy, endoscopy, etc.), and episode summaries (discharge letters). Our special attention concerns the core parts. In paragraph 3.2 we describe two firstgeneration EMR-systems that were explicitly built according to a typical philosophy with respect to the structure of the medical record and that served as a model for later systems. In paragraph 3.3 we discuss the first generation of pragmatic, classical EMR- 
systems that matured before 1990 and are now used in many medical domains. Paragraph 3.4 deals with the new initiatives after 1990 , most of which are still in a pioneering phase. The order in which these initiatives are described is a rough reflection of the number of publications about the subject generated by each research group. In the discussion (paragraph 3.5) we shall summarise the state of the art of three problem areas concerning the use of medical narratives: the facilitation of data entry, the understandability of data, and the facilitation of information retrieval for direct patient care.

\subsection{Two typical systems}

Around 1970 two approaches were advocated to improve the (traditionally sourceoriented) organisation of the medical record, in particular of the narrative parts: the problem-oriented medical recorda introduced by Weed, ${ }^{4}$ and the time-oriented medical record described by Fries." To demonstrate the viability of their ideas, they both developed a computer-based version of their medical-record structure. Both EMRsystems have had a large impact on later developments. Principles of problem- and time-oriented medical record keeping were, to a certain extent, incorporated in most of the computer-based medical-record systems that would be developed in the following decade.

\subsubsection{PROMIS}

The Problem-Oriented Medical Information System (PROMIS) was under development since 1969 at the Medical Center Hospital of Vermont in an attempt to computerise Weed's ideas about how to structure the medical record and how to use medical knowledge for the care of the individual patient. "The physician, when making decisions, was guided by the system in a scrupulous way. For example, it took eight steps to complete a plan for further action. An evaluation of the use of PROMIS for medical audit (another issue in Weed's ideas) was not convincing, however. When comparing PROMIS-generated print-outs to the paper-based problem-oriented medical recond, physicians judged the format of PROMIS inferior, and the expected advantages of PROMIS for audit purposes could not be demonstrated at all. ${ }^{\text {s }}$

Ultimately, the PROMIS-system did not survive, which was attributed, by others, to its "dogmatic nature"." Evidently, the use of PROMIS demanded too much discipline from the physician. We suppose that the pretensions of the developers of PROMIS were a bridge "oo far, given the technical means at that time. The idea of

\footnotetext{
an the problem-oriented medical record, each progress note is related to a problem from a previously defined "problem list" and structured according to four phases of medical reasoning: subjective. observations, objective obserwations, assessment, and plan ("SOAP"). For more details see Chapter 4 or the indicated reference in the text.

b In the lime-oriented medical record, medical history and physical examination are structured into detailed data-types and all medical data can be displayed in a flowsheer (a two-dimensional type $x$ time matrix), hence visualising their evolution in time. For more details see Chapter 4 or the indicated reference in the text.
} 
problem-oriented medical-record keeping, however. would return in many other EMRsystems.

\subsubsection{ARAMS}

The American Rheumatism-Association Medical-Informarion System (ARAMIS) was the computer-based version of Fries" fime-oriented medical reoord, which was originally presented in paper format. In ARAMIS, all observations from medical history and physical examination were recorded as separate items and stored in coded format, by intermediation of paper flowsheets. Medical deliberations were captured in free-text format in the assessment part of the medical record. "In a later version. medical history and physical examination were recorded on encounter forms instead of flowsheets, but it is unclear whether the use of free text in these parts was allowed." Primary purpose of ARAMIS was (and still is) to serve as a national research data bank for the storage and disclosure of longitudinal data of chronic rheumatologic diseases. Many studies have been published about the use of ARAMIS supporting research concerning rheumatologic diseases, e.g., to determine diagnostic criteria, and to explore economic implications, long term outcomes, and (side-)effects of therapies. To further support the research use of ARAMIS, a decision-support system was developed to assist the researcher in the generation of hypotheses and the exploration of data. ${ }^{12}$

Much less has been written about the use of ARAMIS for direct patient care. A paper-based version of the time-oriented nedical record was evaluated with respect to search speed and performed four times as last as the traditional paper-based medical record." It was suggested that for individual patients ARAMIS could be used for searching similar cases in order to determine an overall prognosis and to estimate the outcome of alternative therapies. ${ }^{13} \mathbb{n} 1986$, Downs at al. described an expert system that automatically generated a summary of individual patient cases stored in ARAMLS. ${ }^{14}$ More recently, research has been reported concerning extensions of SQL to facilitate temporal reasoning (i.e., interpreting developments in time), which has resulted in a revival of the interest in the use of ARAMLS at the point of care, as a typical time-oriented EMR-system. ${ }^{15-18}$

\subsection{The classical systems}

In the 1970s and 1980 s several computer-based medical-record systems were introduced that evolved to systems used in many medical disciplines, completely integrated with the hospital-information system, and extended with various kinds of decision support. These systems are still operational, and some of them are used in various institutions.

\footnotetext{
a Standard Query Language (SQL) is a, broadty used, formal language to define, enter, select, and modify structured data in a relational database.
} 


\subsubsection{RMRS}

The development of the Regensirief Medical-Record System (RMRS) at the Veterans Hospital of Indianapolis started in 1972. Distinguishing characteristic of this system is the systematic provision of computer reminders to the users of the system. ${ }^{\text {a The effect }}$ of such reminders on physicians" behaviour and clinical outcome was investigated in several studies. ${ }^{1921}$ From a description in 1988 , it can be concluded that the major parts of medical history, physical examination, and progress notes were not captured in the computer. Narrative reports from auxiliary services were stored in the system as free text, and only a few narrative data were, experimentally, stored in coded format, after initially being captured on pre-structured paper forms. ${ }^{22}$ Such coded narratives could be presented to the reader in fluent prose after being processed by a natural-language generator that collates these expressions using pre-formatted templates. ${ }^{23} \mathrm{McD}$ onald's philosophy was to wait with further computerisation of medical narratives until there would be broadly accepted standard terminologies and ready-to-use technologies for voice recognition and natural-language interpretation. In the mean time, he assumed, the "paperless medical office" would be an utopia. ${ }^{24}$ This attitude was criticised by Korpman, who argued that since the main function of the medical record is to support the communication between health-care providers, the complete capturing of medical narratives was most urgent, even if these were in the format of unstructured notes. ${ }^{25}$

From a description of RMRS in 1992, it can be inferred that the capture and storage of medical narratives in RMRS had not changed fundamentally. Only in the domain of obstetrics, a complete capture of coded medical narratives was achieved, on prestructured forms, which were entered in the computer by clerks. In other domains, medical narratives were partly dictated and transferred to the computer as free text (usually manually, but some reports already through automated voice recognition), and partly written on paper charts and not stored in the computer ${ }^{26}$

\subsubsection{STOR}

The Summary Time -Oriented Record (STOR), the development of which started in the late 70s, was operational hospital wide in San Francisco in 1985. The system"s name reflects the underlying philosophy to store only a summary of the medical narratives in the computer. In a description from 1988, physicians were said to use pre-structured forms to record a brief selection of controlled terms from medical history and physical examination, in combination with a global frec-text impression of progress and plan. This information was subsequently entered into the computer by clerks. In about $25 \%$ of all outpatient visits it was necessary to keep a complementary paper medical record to store additional medical narratives. ${ }^{27}$ The STOR-system has been evaluated in an elegant way. Before the patient encounter, physicians were asked to predict the presence of symptoms and the results of laboratory tests on the basis of available information from either the paper medical record, STOR, or a combination of both. The physicians" predictive power when using STOR alone did not differ from that

a Computer reminders are messages containing recommendations to the physician about actions that should or should not be performed (preventive measures, contra-indicated drugs, etc.) 
when using the combination of STOR and a paper medical record. Both appeared to be superior to using a paper medical record alone.

\section{3 .3 HELP}

The HELP medical-record system, operational at the LDS Hospital in Salt Lake City since 1975, was designed around the patient care process from the beginning. Its distinguishing characteristic is the emphasis on decision support, e.g, wo direct the respiratory therapy of intensive care patients. ${ }^{30}$ In $1988,50 \%$ of all "subjective" medical data was captured in the computer, almost entirely stored in coded format. "It is not clear from the literature, to which extent medical nartatives (in our definition) belonged to the computer-stored part. Data were entered into the computer using preformatted, but customisable, screens and (proprietary) controlled vocabularies of medical terms.a Recently, a new method to facilitate data entry was developed. This method, called Pick from thousand (PFT), is a combination of keyword matching, minimal matching, and wild-card matching. Benchnark tests were promising, and a clinical evaluation was reported to be in progress. ${ }^{32}$ Other recent developments concerning the HELP-system concerned the transition of the HELP-system to a more open architecture, ${ }^{33-35}$ and a further extension (including evaluation) of the decisionsupport functions of the system. ${ }^{36-39}$

\subsubsection{TMR}

The Medical Record (TMR), operational at Duke University Medical Center since 1970 , is perhaps the most comprehensive of all classical systems. Already in 1983, it was designated as a "chartless" medical record." In 1988, only images were not included. Narrative data were all captured, either as free text or (what was encouraged) in coded format using proprietary controlled vocabularies. A report generator collated these coded data into narrative text documents, resulting in natural-language output. The lack of a standard vocabulary was considered a major problem with a high priority to be solved. "In 1992, also the development of new methods for data entry got high priority on the research agenda, since physicians were still reluctant to interact with TMR directly. ${ }^{22}$ Data entry into the computer took more time than via paper, and humans were better in browsing through information when presented on paper documents than when presented on a computer monitor. Hence, most of the data had to be entered by special data-ferminal operators (DTO"s). To encourage direct data entry by physicians, electronic mail facilities were offered for the recording of various narrative reports. For the future, the best structule to facilitate the retrieval of information by physicians was considered something "very much like an expandable roadmap", with hypertext links for accessing more detailed in lormation."

\footnotetext{
a A controlled medical vocabulary or medical terminology is a standard, structured, list of medical terms from which the user can select for describing his observations, etc. A controlled vocabulary may be proprietary or open. Proprietary vocabularies are exclusive for one department, institution, or vendor, and can be easily accommodated. Open vocabularies are shared among enterprises, even across nations, and their standards are supported by broad consensus.
} 


\section{3 .5 COSTAR}

COSTAR, developed between 1968 and 1978 at Harvard Medical School, was designated in 1988 as the most widely used computer-based medical-record system for ambulatory care. ${ }^{6}$ In 1984 , medical narratives were recorded either on predefined paper encounter forms or by audio-tape, and subsequently entered into the computer by clerical personnel. Most narratives were captured as free text, while problems and diagnoses were stored in coded format with the use of user-definable lexicons (i.e., proprietary controlled vocabularies). ${ }^{44}$ In more recent publications we did not find evidence of substantial changes in the capture of medical narratives. The system was also used in a primary care setting. Here, $80 \%$ of the medical narratives was captured by check-offs of pre-coded items. ${ }^{45}$ Recent implementations of COSTAR induced a mixture of enthusiasm and, especially among infrequent users, resistance. ${ }^{6.47} \mathrm{~A}$ promising experiment with a revised physician-computer interface for direct physician input, more similar to the familiar paper chart than to the original COSTAR-interface, was conducted by Dell et al. and resulted in a doubling of the frequency of direct physicians" use.

\subsubsection{Beth Israel}

The Beth-Israel Hospital in Boston has experience with the use of an integrated hospital-information system since the late 70 s. Initially, developments concentrated on facilitating clinical research. ${ }^{49.50}$ Subsequently, the focus of development concerned direct computer support at the point of care. In 1989, a computer-based outpatient medical record became operational for use both inside and outside the hospital. The aim was to eliminate the use of paper forms for data entry in favour of direct physician-computer interaction. Dictation and subsequent transcription was not encouraged, though still allowed. Of the medical narratives only progress notes were included, written and stored as plain free text. Each progress note could be related to one or more problems, which (in the hope to enhance the acceptance of the system) could also be defined in free text. On the basis of these free-text problem descriptions, a dynamic data dictionary of problems was maintained off-line, to facilitate clinical research and problem-based decision support. A retrospective evaluation of the use of the medical-record system showed, that physicians were more inclined to maintain a problem list when using the computer-based medical record than when using a paperbased version. Also, the computer system saved time during follow-up encounters and telephone consultations. ${ }^{51}$ Recently, the computer-based medical-record system was extended with an active decision-support system that generates alerts and reminders based on computer-stored practice guidelines (protocols) for the treatment of HIVpatients in the ambulatory setting. ${ }^{52-54}$

\subsubsection{DIOGENE}

DIOGENE is an integrated hospital-information system in Europe with a long tradition of research and development. Originally, narrative patient information was entered by a telephone call to an operator pool of data entrists. The text was typed by an operator and immediately visualised on the physician's or nurse's clinical workstation, hence 
enabling interactive corrections when necessary ${ }^{35}$ More recently, three alternative strategies have been explored concening the capture of medical narratives.

Assimacopoulos et al. described a module for the capturing of free-text problemoriented progress notes. Each note was indexed with a unique identifying term and could be linked to a problem from a pre-defined problem list. Main purpose of this module was to make progress notes better accessible for reviewing medical decisions. ${ }^{56}$ A variant of this approach, in which progress notes were structured in four sections (problem, objectives, actions, evaluation), was later used for nursing notes, Evaluation was planned but not reported. This approach of structuring free-text documents resulted in UNIDOC, a document-management system accessible from PCworkstations using MS-Word ${ }^{\mathrm{TM}}$. In UNIDOC, searching medical narratives is possible by using indexed paragraphs as well as by full-text retrieval functions. ${ }^{58.59}$ The support of progress notes by UNIDOC has been temporarily abandoned in favour of the support of summarised reports, such as discharge letters and radiology reports. ${ }^{60}$

A second approach to the capture of medical data is the registration of follow-up observations in a time-oriented flowsheet, described by Bolens et al. Purpose was to make all medical observations directly accessible by type and time, independent of any diagnostic interpretation. All observations were scored separately, as normal or abnormal. For each consult, also a global indicator of the severity of the patient state was recorded. It was demonstrated that this way of documentation contained valuable information for retrospective studies. ${ }^{61}$

The third and most comprehensive area of research on the capture of medical narratives concerns natural-language interpretation. Starting from the assumption that narrative information can best be presented as free text, Baud et al. described a project that should lead to a system able to (a) analyse physician-entered free-text notes, (b) generate a structured representation of this information for query-purposes, and (c) generate free text notes from a structured representation. The system should be robust against syntactic violations (such as the telegraphic style of progress notes), and useful for different European languages. ${ }^{62}$ Initially, only discharge summaries were used to feed the system. ${ }^{62}$ In a more recent description of this system (named RFCIT) given by Rassinoux et al., medical free text is interpreted both syntactically and semantically, and represented as conceptual graphs. ${ }^{63}$ The system was adapted to make use of the GALEN-terminology-base for the semantic analysis of medical expressions. ${ }^{64}$ This support was exactly what the RECIT-system needed: to check the semantic compatibility of free-text phrases, even when syntactically odd. An evaluation of RECIT has not yet been reported.

\subsection{New initiatives}

The problems with medical narratives that were identified in publications about the classical systems were picked up by research groups that did not (yet) have to maintain their own operational system. Most initiatives resulted in prototype systems, applied in only one or two medical domains. The stage of operational use in and integration with the hospital-information system has generally not yet been reached. 


\subsection{Stanford}

Already in 1985, the Section of Medical Informatics of Stanford University reported a study about structured data entry by physicians in an electronic medical-record system (ONCOCIN). ${ }^{65}$ Their recent research activities related to the computer-based medical record are multi-faceted. Most of these initiatives are deployed under the umbrella of the Therapy)-Helper project, which aims to develop an electronic medical-record system that should facilitate protocol-based care of HIV-patients.

Musen et al. described the creation of a proprietary controlled vocabulary for the treatment of AIDS, to be used in the T-Helper system. This work turned out to be very laborious, even for such a small-scaled domain. ${ }^{66}$ Campbell et al. examined the alternative of using a standard vocabulary for this purpose, namely SNOMED-III. ${ }^{67}$ SNOMED-III was chosen because it is expressive, relatively domain complete, and because it can be extended with modifiers and special time references. ${ }^{68}$ Because SNOMED-III does not provide a syntax to validate sensible combinations of terms, Campbell cleveloped a consistency checker, formalised in conceptual graphs, that should support the generation of complex terms in SNOMED. ${ }^{69}$ Conceptual graphs are a graphical notation for representing logical expressions, as a more intuitive alternative of mathematical expressions. ${ }^{70}$

For the user interface of the T-Helper system, called Nory, two different ways of entering narrative data are under study. $Q$-Med is a data-entry module for recording medical history by speech recognition and subsequently interpreting and converting these utterances into controlled expressions. The quality of data gathered by Q-Med was evaluated by Johnson et al., who found a semantic accuracy (recall) of $85 \%$ and a false positive rate (1-precision) of $10 \%$, for open-ended questions. ${ }^{\text {"1 }}$ PEN-Ivory is a data entry module that supports pen-based entry of structured data for the subjective and objective sections of progress notes. Alternative prototypes of PEN-Ivory, differing in three aspects of user interface design, were evaluated on speed of data entry by Poon et al. ${ }^{72}$ These aspects were: scrolling versus paging panels, a dynamic versus a static palette of expression modifiers, and a context-sensitive subset versus a complete set of data items to select from. For all three aspects, the alternative with the most stable screen design scored best (viz., paging panels, a static palette of modifiers, and a complete set of items). Data entered via PEN-Ivory are stored as SNOMED-III code. A text generator provides fluent prose when presenting these data. ${ }^{73}$ The presented progress notes have a SOAP-structure (subjective observations, objective observations, assessment, plan), but are not problem-oriented. The subjective and objective sections are partitioned by organ system, inside which paragraphs the information is presented as fluent prose.

Another project of this group led to the CAPIS system. ${ }^{74}$ Aim of this project was to develop an algorithm-driven language-processing method for the extraction of physical-examination findings from free-text discharge summaries. Before all, this method had to be simple, without the need for heavy investments for developing domain-specific knowledge bases. Compared with the results of manual extraction from 20 discharge summaries of gastrointestinal-bleeding cases, CAPIS recalled $95 \%$ 
of the relevant findings, with a precision of $96 \%$. The natural-language interpreter developed for CAPIS was the same as was later used within Q-Med."

\subsubsection{Manchester}

A long term project of the Medical-Informatics Group in Manchester (UK) is the development of the PEN\&PAD clinical workstation. The main goal of this project is to develop a clinical workstation for physicians, nurses, and other health-care providers that enables the capture of any information that is medically sensible in a structured way. Primary function of the PEN\&PAD-system is to support direct clinical care, hence the system should be simple to use and at the same time be capable of dealing with the complexity of detailed (mostly narrative) clinical information. Part of the PEN\&PAD-project was to reconsider the fundamental principles behind the medical record ${ }^{75}$ The medical record should be "faithful" to the physician's observations, to the decision-making process, and to the clinical dialogue (which comprises requests, referrals, opinions, and the corresponding responses). It should consist of "what clinicians have stated about what they have heard, seen, thought, and done". With other words, it should retain what clinicians believe, which is not necessarily the same as what is true about the patient.

This concept of faithfulness resulted in the design of a medical record represented as a sophisticated network of nested objects, forming the basis for the PEN\&PADworkstation. ${ }^{76}$ One of the underlying premises was that each entry should result in controlled expressions, i.e., that all data should be constructed from terms selected from a controlled medical vocabulary. ${ }^{77}$ A first prototype of this workstation was made for general practitioners, ${ }^{78}$ a second and third for physicians and nurses respectively in an elderly-care ward. ${ }^{79}$ One of the most distinguishing characteristics of the PEN\&PAD workstation is the function of predictive data entry ${ }^{80}$ Data entry is guided by structured, context-dependent, forms that are composed dynamically. The composition of these forms is supported by a special formalism, first called Structured Meta-Knowledge (SMK), later known as Galen Representation And Inference Language (GRAIL). Although originally developed to support predictive data entry, GRAIL became also the vehicle to specify the content of the GALEN medical terminology-base. ${ }^{64.81}$ GALEN represents medical concepts independent of any natural language and includes consistency-checks on combinations of terms.

In the PEN\&PAD user-interface, medical narratives are presented as a number of separate items, following the same outline structure as the paper medical record. The user can easily switch between different presentation formats, such as a summary form and foldable flowsheets of progress notes, laboratory results, medication, etc. Heathfield et al. evaluated the PEN\&PAD user-interface, in a small simulative setting, in the domain of elderly care. The test persons (ten nurses) were enthusiastic about the information retrieval capabilities of the system, but not really satisfied with the items that were proposed by the predictive data-entry function. It was suggested, that the less positive response to predictive data entry might be attributed to the less structured way nurses work, compared with physicians. ${ }^{79}$

Recently, Kay et al. proposed a completely and contrasting new approach to the design of a faithful medical record, founded in narratologic theories. ${ }^{82}$ They argued, 
that clinical decision making is a kind of narrative reasoning, and that medical narratives can be seen as "stories" that are interpreted by both the author and reader from their own contextual framework. Natural prose is the most convenient way to enter such stories. Hence, in contrast with the premises underlying the PEN\&PADworkstation, they suggested for the future a combination of free-text data entry and natural-language interpretation techniques.

\subsubsection{Rotterdam}

At Rotterdam Erasmus University, a medical workstation has been developed (called HERMES) that was originally meant to be used for research purposes as a user interface that gives access to information from different sources (e.g., legacy information systems in the hospital) and to standard statistical software. ${ }^{83}$ A variant of this system (recently named ORCA) was prepared as an electronic medical-record system for direct patient care. Main philosophy behind ORCA is to stimulate (rather than urge) the physician to enter narrative data in a structured way. The physician is stimulated to enter structured data in a discipline-specific sub-record, but may also enter his narratives as free text in the medical mother-record. ${ }^{84}$

The narrative part of the mother-record consists of free-text bodies of medical history, physical examination, and (problem-oriented) progress notes. The physician can express his insights by placing indication and motivation links between different text bodies. The sub-record supports two ways of capturing structured domain-specific data. Highly predictable data are entered via pre-defined, structured, forms. The order in which these forms are presented is guided by a State Transfer Monitor (STM) that contains knowledge about the use of forms per discipline. ${ }^{85}$ The capture of domainspecific data with low predictability, on the other hand, is directed dynamically by context-dependent menus. These menus are composed automatically with the help of a detailed domain-specific knowledge base, which is represented as a semantic network of medical concepts and their relation to one another. When the physician is describing, for example, endoscopic findings of the stomach, the menus are filled with only those concepts that are sensible in the given context. ${ }^{86}$

ORCA is still a system under development. The domain-specific knowledge base of the mother-record does not yet include synonyms and cannot yet express the physician's uncertainty. The sub-record for highly predictive data has been applied in the domains of cardiac failure and andrology. In the latter domain it was part of a system developed to access clinical data from multiple legacy systems for research purposes. ${ }^{87}$ The sub-record for data with low predictability was studied in the domain of endoscopic procedures. It was reported, that endoscopic reports created with the help of this sub-record were more complete than when entered as free text. ${ }^{88}$

\subsubsection{New York}

Relevant research at Presbyterian Medical Center of Columbia University concerns mainly the use and further development of a Medical-Entity Dictionary (MED) derived from the UMLSS, ${ }^{89}$ and the use of Internet protocols and browsers as an environment for rapid application development of modules for medical workstations that have to 
communicate with legacy clinical-information systems as well as with external information sources (like Medline ${ }^{\text {TM }}$ ). ${ }^{90}$

Further, Barrows et al. described a model of an electronic medical-record system that supports the monitoring of clinical reasoning. In this system, each free-text progress note is SOAP-partitioned and indexed to a problemwate, which is a temporary assessment of a problem. A chain of problem-states reflects the evolution of clinical understanding with respect to a certain problem. In 1994, while the system was in its final testing phase, the model was only implemented in part, due to "logistics of the user interface". Not implemented, e.g., was the association of progress notes with explanatory observations. "Note, that Barrow's problem-states emphasise the dynarnic character of problem formulation. A similar dynamic concept was present in Assimacopoulos" progress notes indexed with unique identifiers. ${ }^{56}$

\subsubsection{Other initiatives directed to the capture of medical history. physical examina- tion, and progress notes}

Rather provocative critique on the approach of capturing medical narratives as coded data was given by Lincoln and Essin. Main concern of these authors is the problem that the use of controlled expressions (which they consider as a typical exponent of the usual solution-driven way of system engineering in medical informatics) results in rigid data models. ${ }^{92.99}$ Such data models "ignore one fundamental requirement: the abulity to deal with the unexpected" and hence are unable to cope with changing demands of physicians. They propose a medical record architecture consisting of loosely-structured documents: loose in its free-text content, structured in its outlines. They suggest the use of standard general mark-up language (SGML) for constructing these outlines. As an important advantage of their approach they consider the fact that it enables physicians to gradually structure the medical record to the desired level of detail. ${ }^{94}$ The recent state of Lincoln's and Essin's work is unclear. On one hand, they persist in advocating the tagged-text approach, however without giving evidence that (even a prototype) implementation exists. ${ }^{95}$ On the other hand, they seem to have abandoned this philosophy in a publication in which they present a prototype low budget computer-based medical-record system that is based on the (criticised) application of a relational database and a controlled vocabulary. ${ }^{96}$

Trace et al. described the user-interface of IMR-E, a medical-record system to facilitate both data entry and information retrieval. This system enables context. sensitive entry of structured data which is supported by a knowledge base of medical terminology. This terminology includes specific information about 105 complaints as well as the complete ICD-9 dictionary, which is used as vocabulary for diagnoses and as an index to specific clinical guidelines. The entry of topographic information is facilitated by pointing to anatomic pictures. Observational data from medical history and progress notes can be presented in natural prose. Progress notes are SOAPstructured. Details about other aspects of the IMR-E system, such as the architecture of

a SGML (standard general mark-up language) is a general syntax for formatting free-text documents. A well-known document format defined with SGML is HTML (hypertext mark-up language), which is used for generating pages for World-Wide-Web browsers. 
the system and its knowledge base, its application domain, and evaluation results, were not reported:

Nygren et al. observed seven physicians from different specialties when reading their inpatient medical records. They found that physicians used three reading techniques: skipping over irrelevant sections, skimming through possibly relevant passages, and reading carefully the information needed. They considered that these reading skills are an integral part of diagnostic expertise. Therefore, their observations should have implications for the design of the user-interface of the electronic medical record. $\%$ They developed a prototype user interface in which free-text medical narratives are presented as exact imitations of paper documents. Hence, the physician is still able to use his usual reading skills. The user can obtain information in three steps: first he selects the main compartment (the source of information: physician, $X$ rays, laboratory, etc.) by clicking the appropriate icon, second he selects the specific document by scrolling through an index, and third he can read the content of this document. Document reading is facilitated by the use of linearly-structured free text (instead of hypertext) and the use of paging windows (instead of scrolling). Both tradeoffs were motivated by referring to the users' preference of visual cues at fixed positions on the screen (so-called fixed-position cues) which enable them to observe in a glance, by pattern recognition. whether a certain place on the screen is empty or not. ${ }^{99}$

\subsubsection{Other initiatives directed to the capture of specific reports or summaries}

Much pioneering work on natural-language interpretation was carried out in New York by Sager et al. in the Linguistic-String Project (LSP). Sager and her team developed a medical-language processor (MLP) for converting free-text phrases (from medical narratives) into controlled terms stored in a relational database, which makes them accessible for structured queries, e.g. for review or decision support. The MLP-system makes use of a combination of syntactical and semantical analyses, supported by a controlled vocabulary of medical terms. ${ }^{100}$ Sager has been involved in a pilot project preceding the development of the RECIT-system in Geneva. In this project, 139 freetext discharge summaries (of unknown specialties) were classified at three levels of semantical complexity, both automatically (by the MLP-system) and manually (by physicians). A $72 \%$ agreement was found between both analyses. ${ }^{63,101}$ The MLPsystem was also used in Glasgow to analyse 59 free-text hospital-discharge summaries of asthma patients for evidence of adherence to medical guidelines. Compared with a manual procedure to extract this information, $9 \%$ of the evidence was missed by the MLP-system (1-recall) and $1.7 \%$ was wrongly interpreted as evidence (false positive). ${ }^{102}$

Bell et al. presented a system that supports the structured entry of data for ultrasound reports using predefined forms. The user can choose between keyboard and voice input to select the items to capture. The physicians involved in this project (radiologists and gynaecologists) preferred an outline report that was divided into separate observations to a report in natural language. ${ }^{103}$

Bernauer experimented with context-sensitive, knowledge-base supported, entry of structured data for bone-scan reports. He used a proprietary controlled vocabulary in 
combination with conceptual graphs constraining the generation of combinations of terms. Hence, senseless combinations of terms are ruled out, leaving only contextrelevant options for selection. He developed a report generator to present structured data as free text. ${ }^{104}$

Gouveia-Oliveira et al. described an information system for gastrointestimal endoscopy reporting called SISCOPE, which supports menu-driven context-sensitive entry of structured data. These data are stored in coded format and can be analysed for research purposes. The reports themselves are presented in natural language. Both data entry and report generation are supported by a domain-specific controlled vocabulary. ${ }^{105}$ In an evaluation study, the quality of these computer-generated reports appeared superior to the quality of conventional free-text reports. ${ }^{106}$

In another sub-domain of gastro-enterology (sonography of the upper abdomen), Kuhn et al. developed a reporting system based on the entry of structured data through predefined forms and compared its output with dictated reports, using various criteria. Use of the system resulted in faster data entry and a decrease of omissions; interobserver variability of stored data was low and user acceptance high. ${ }^{107}$

\subsection{Discussion \\ 1)}

The management of medical narratives in the classical systems, though identified as a major concern by many of those who maintain these systenns, did not change significantly after the publication of the book The computer-based patient record in 1991." Most of these classical systems still cannot be used without additional paper medical record, and none of them is exclusively used for direct input by physicians. Only in a few centres some experiments with direct data entry have been performed, inwolving HELP and COSTAR. ${ }^{32,48}$ Presumably, the burden of keeping an integrated system operational for a broad range of medical domains, and keeping it technically up-to-date (downscaling, conversion from hierarchical to relational database structure, etc. $)^{33}$ did not leave much effort to spend on experimental activities. An exception must be made for D[OGENE, which is still in the front-Jine of research and development. The systems that resulted from the later initiatives, on the other hand, are all designed to face the challenge of involving the physician in direct interaction with the computer for the capture and use of medical narratives.

Challenges are being addressed in three problem areas: (1) the understandability of data, (2) the facilitation of data entry, and (3) the facilitation of information retricval. Here, we shall discuss the state of the art with respect to each of these aspects, as far as it can be reconstructed from the reviewed literature. We only take into account the reviewed publications about systems that have reached the stage of implementation and that also include medical history, physical examination, and/or progress notes, hence disregarding systems that were only used to generate or interpret discharge letters or laboratory reports, and also disregarding publications about design matters only. We divide the reviewed systems in operational systems (the classical generation) and experimental systems (mostly, but not exclusively, products of new initiatives). 
Table 3-1:

Formats in which medical narratives are stored. Only implemented systems that are used for the capture of medical history, physical examination and/or progress notes are counted.

Note: Natural language interpretalion converts stored text into controlled expressions. Both formats are permanently stored.

\begin{tabular}{lll}
\hline Format of data storage & Operational use & Experimental use \\
\hline as free text only (FT) & 51,55 & 56,99 \\
as controlled expressions only (CE) & 7,10 & $61,72,80,97$ \\
partly as FT, partly as CE & $22,27,41,44$ & 85 \\
both as FT and, after natural language-interpretation, as CE & 62,71 \\
\hline
\end{tabular}

The numbers refer to references in the text

The picture we provide may be incomplete, because the reviewed literature was sometimes fragmentary, especially concerning the facilitation of information retrieval.

\subsubsection{How to enhance the wnderstandability of data?}

Natural prose is the typical way to express ones observations and thoughts. It does not guarantee, however, that the reader will understand this information exactly as it was meant by the author ${ }^{82}$ The only way to reach unambiguous understandability of medical narratives is to store them as controlled expressions, with the help of a controlled vocabulary. The advantages of unambiguous understandability have been emphasised repeatedly. $10,24,31,77,108,109$ It would facilitate the exchange of medical data between health-care providers, enable context-dependent decision support, make the medical record accessible for statistical analyses, etc. On the other hand, coding medical narratives has also an associated cost: the inevitable loss of nuanced information and of the ability to deal with the unexpected. ${ }^{94.10}$ This makes fully coded medical narratives less flexible and expressive than free-text narratives, a limitation that is most felt when supporting medical reasoning at the point of care.

In Table 3-1, we provide a breakdown of different approaches of storing medical narratives in electronic medical-record systems. Among the operational systems, the dominant approach is pragmatic: they use controlled expressions where possible and free-text storage otherwise. The developers of experimental systems, on the other hand, opt for a more idealistic approach of banning out free-text storage. The reason why the latter have more faith in the exclusive use of controlled expressions might be youthful carelessness, due to a lack of experience in maintaining an EMR in an operational environment with a broad range of medical domains. On the other hand, the presently available controlled vocabularies have a higher expressive power and are adopted by more users than before. In particular, SNOMED-III and GALEN are promising vocabularies that are open, relatively domain complete, and able to represent complex medical terms. ${ }^{64.67 .811}$ GALEN has an intrinsic mechanism of determining medically sensible expressions. Campbell et al. showed, that SNOMED-III can be also extended with such a mechanism by the use of conceptual graphs. ${ }^{68.69}$ On the other hand, although open standards are protected against incidental modification, the standards themselves are subject to more or less frequent updates. A standard is the result of a negotiation process between people with different and often conflicting interests and 
Table $3-2$

Methods for the entry of medical narratives. Only implemented systems that are used for the capture of medical history, physical examination, and/or progress notes are counted.

\begin{tabular}{lll}
\hline Method of data entry & Operational use & Experimental use \\
\hline - For all medical narratives & & \\
indirect (transcribed) & $10,22,27,43,44,55$ & 61 \\
direct... & 7,31 & $32,48,51,80,85,97$ \\
... via keyboard or mouse device & 26 & 71 \\
.... vice recognition & & 72 \\
\hline
\end{tabular}

- For controlled expressions only

static entry

$7,10,22,27,31 \quad 61,85$

dynamic entry

natural-language interpretation

The numbers refer to references in the text

eeds, which makes each upgrade difficult to achieve. ${ }^{112}$ And when achieved, the upgrade of a vocabulary in an existing medical-record system may jeopardise the historical consistency of previously entered patient data. Cimino illustrated that keeping the medical-record database consistent, after a terminology upgrade, is not a trivial exercise. ${ }^{89}$

Conversion of medical narratives entered as free text into controlled expressions, supported by natural-language interpretation, is only performed in two experimental systems. This way of handling medical narratives is regarded to play a major role in the future. ${ }^{1.24 .82}$ For the moment, however, this method is (apart from the two studies mentioned in Table 3-1) mainly tried out on discharge summaries in some welldescribed domains. ${ }^{74.101 .102}$

\subsubsection{How to facilitate the entry of data?}

Some years ago, the reluctance of physicians to record medical narratives in direct interaction with the computer was recognised as a major impediment for the acceptance and use of EMR-systems. ${ }^{1.24 .32 .43 .51}$ As possible future technical solutions were suggested the use of hand-held terminals and techniques for voice recognition, ${ }^{1.24}$ Table 3-2 show/s that, in general, direct entry of medical narratives by the physician is still in the experimental phase. Illustrative is the fact that the Stanford research group experimented with two different methods for data entry: hand-held terminals in PENIvory, and voice recognition in Q-Med. ${ }^{7.72}$

The entry of medical narratives for storage as controlled expressions faces some specific problems. The challenge is here, how to trade-off maximal control, to assure the quality of input, against maximal flexibility, to accommodate the individual patient case. Three methods have been employed. The oldest method is to guide data entry by the use of static, predefined encounter-forms, either on paper or on screen. This way of data entry is suitable for restricted, well-defined medical sub-domains with a predictable, patient-independent, pattern of information needs (e.g.., nephrology, 
orthopaedic surgery). In other medical domains, the pattern of data entry is less predictable beforehand (c.g., general internal medicine). This requires a more dynamic type of data entry in which the context of previously recorded information has to determine the options for further data entry. Such support is realised by providing accommodative entry forrns or menu options, displaying only those terms that make sensible expressions in combination with previous input. The combination of free-text data entry and natural language processing for obtaining controlled expressions is the most fexible solution. Medical narratives are stored provisionally as free text, while the conversion to controlled expressions is postponed to a later phase. The experience with this method is growing rapidly and the quality of generated data is carefully monitored. However, as long as the conversion into controlled expressions does not take place immediately during data entry, it is not possible to generate and use on-lline reminders and alerts.

\subsubsection{How to facilitate the retrieval of information?}

The entry and storage of medical narratives cannot be a goal as such. It is a necessary prerequisite for the many uses of these data. The primary function of the medical record is to support direct medical practice, which requires that the physician should be able to access all information he needs quickly. We encounter only a few studies to the facilitation of information retrieval from medical narratives. ${ }^{1099}$ From the description of many systems, we deduced three kinds of measures that were taken to improve the accessibility of medical narratives:

1. Presentation of medical narratives as fluent prose to facilitate reading - Many EMR-systems in this review present their narratives in fluent prose, even if they have to generate it from coded data. $23,41,72,97,104,105$

2. Optimisation of the screen design by the use of fixed position cues - Two publications paid considerable attention to the design of the user interface. ${ }^{72.99}$ They both indicated the added value of fixed position cues to guide the physician when searching through the mass of data. The user-interface should support paging rather than scrolling panels, static rather than dynamic (context-sensitive) selections of options and data items, and linearly structured text rather than a labyrinth of hypertext links. In short, for presenting medical narratives the interface should be simple and stable. It should be noted, that the need for fixed position cues seems contrary to the need for accommodative forms and menus, which are used to support dynamic data entry. Perhaps, the less positive experience of nurses with the PEN\&PAD predictive data-entry function was due to the unpredictability of the screen content. ${ }^{79}$ Also remarkable is the objection against the use of hypertext in a time that this phenomenon has assumed enormous proportions on the World-WideWeb. Hypertext links can be encountered anywhere in a document, which does not enable the user to develop standard patterns for searching. ${ }^{99}$

3. Imposing a searching structure on medical narratives - Important principles for the organisation of medical narratives were proposed in the problem-oriented and time-oriented medical records $s^{4.5}$ and first used in PROMIS and ARAMIS respectively. ${ }^{3,10}$ Although these principles also effect the entry and storage of data, their primary purpose was to facilitate the retrieval of information. In the problem- 
Tabie 3-3:

Aspects of the searching structure of medical narratives. Only implemented systems that are used for the capture of medical history, physical examination, and/or progress notes are counted. Incomplete picture due to fragmentary evidence.

\begin{tabular}{|c|c|c|}
\hline Gramularity of medical narratives & Operational use & Experimentat use \\
\hline \multicolumn{3}{|l|}{ - Progress notes } \\
\hline problem-partitioning & 9 (several mentioned) & 85,91 \\
\hline SOAP-partitioning & 7 & $57,71,72,97$ \\
\hline \multicolumn{3}{|l|}{ - Medical history / physical examination } \\
\hline undivided & 40,51 & 85 \\
\hline partitioned by organ sy'stems & & 71.72 .99 \\
\hline partitioned by separate observations & 10 & $61,79,85$ \\
\hline Perspectives of medical narratives & Operational use & Experimentaluse \\
\hline source-oriented flowsheets & 9 (several mentioned) & \\
\hline problem-oriented flowsheets & 9 (several mentioned) & \\
\hline mativation or indication links & 7 & \\
\hline
\end{tabular}

The numbers refer to references in the text

oriented record, the progress report is divided into problem-related sections, and each progress note is partitioned into SOAP-sections. In the time-oriented record, medical history and physical examination are partitioned into paragraphs corresponding with separate observations and the evolution of medical data over time is displayed in flowsheets. Progress noles, in this case, do not contain observations any more but only assessment and plan. A combination of problemand time-oriented principles has been realised in a problem-oriented llowsheet, which displays the evolution of problem-related data over time, and which is commonly used in most operational, classical, systems. "The searching structure of medical narratives has two aspects: the granularity of the paragraphs in which these parts are divided and the way in which these paragraphs are related to one another, i.e., the perspectives from which these paragraphs can be accessed.

Table $3-3$ provides an overview of how medical narratives are structured for presentation. It should be noted, that this overview is incomplete, because many authors were not explicit in their description of the presentation structure. For example, there was little evidence in the more recent literature of the use of problem-partitioning and problem-oriented flowsheets. We assume, however, that these structures still occur. SOAP-partitioning, on the other hand, has never become popular. For a long time, Weed's own PROMIS was the only electronic medical-record system in which SOAP was implemented. However, the need for insight in the process of medical practice is growing.' From one system (Ivory) we know that it includes SOAPpartitioned progress notes without problem partitioning. ${ }^{71,72}$ Different elements of SOAP-partitioned progress notes can be linked to one another in order to express a motivation, an indication, or other valuable information about the clinical decision process. We found two examples in which this possibility was worked out in the data 
model, but nether of these were implemented in the user-interface ${ }^{85}$ Also with respect to the granularity of medical history and physical examination the system developers follow different directions. We found that medical history and physical examination are presented in three different granularities: (1) without partitioning, 40.49 .55 (2) with paragraphs on the level of organ systems, ${ }^{90.113}$ (3) partitioned into separate observations or even single findings. $61,79.85$ One system (Ivory, again) chose for a presentation in which observations are stored as single findings, collated into paragraphs at the level of organ systems (review of systems, anatomic regions), and subsequently converted to natural prose. ${ }^{71.72}$ The reason why the structure of medical narratives was collated to organ-system level when being presented, was not given. In general, it can be said that we did not encounter any evaluative study of the searching structure.

\subsection{Conclusion}

The classical systems seem to be subject to the dialectics of progress, at least with respect to the way they manage medical narratives. Their many users demand operational continuity, and their dated architecture (hardware dependence, hierarchical database structure) is also an obstacle for continuous innovation. The recently developed systems have been designed specifically to support innovative approaches to the capture of medical narratives, but are still experimental. They have not yet been evaluated in an operational environment, nor were they applied in a broad range of medical domains. Hence, it is too early to assess the value of these innovations in daily medical practice.

Recent initiatives have put most effort in obtaining unambiguous understandability of medical narratives. Two innovative approaches for obtaining controlled expressions have been tried: dynamic data entry and natural-language interpretation of free-text entered data. Both these approaches need an appropriate controlled vocabulary. Although recent developments of standard and highly expressive controlled medical vocabularies are promising, they are not mature yet and the necessary process of continuous upgrading seems cumbersome. Initiatives to facilitate the entry of narrative data have focused on the control rather than the ease of data entry. Advanced input devices that could make data entry easier, such as voice-recognition systems and handheld teminals, are not used extensively yet.

Several measures have been described to enhance the physician's capability of retricving narrative information for direct patient care. First, it is widely accepted that medical narratives are best presented in natural prose, even if such prose has to be (re)generated from coded format. Second, it has been demonstrated that searching is faster when using a simple and stable user-interface with fixed position cues. Third, organising principles like source-oriented and problem-oriented flowsheets are used to facilitate the physician when searching paragraphs that contain relevant information. The granularity of paragraphs in existing systems varies. The added value of a searching structure and the effect of the granularity of paragraphs used to present 
medical narratives have not been evaluated yet. Further research in this direchion is recommended.

\subsection{References}

1. Dick RS, Stein EB. The computer-based patient record - an essential technology for health care. Washington DC: National Academy Press, 1991.

2. Slack WV, Hicks GP, Reed CE, Van Cura L. A computer-based medical-history system. N Eng $\downarrow$ J Med 1966;274:194-198.

3. What is an Electronic Patient Record? World Wide Web: Medical Records Institute, $1995 \mathrm{htpm} / /$ www.medrecinst.com/mri/levels.html.

4. Weed LL. Medical records that guide and teach. N Engl J Med 1968; 278:593-600,652-657.

5. Fries JF. Alternatives in medical record formats. Med Care 1974; 12:871-81.

6. McDonald CJ. Computer-stored medical record systems [editorial]. MD Comput 1988; 5:4-5.

7. PROMIS-Laboratory. Representation of medical knowledge and PROMIS. Proc Annu Symp Comput Appl Med Care 1978:368-400.

8. Stratmann WC, Goldberg AS, Haugh LD. The utility for audit of manual and computerized problem-oriented medical record systems. Health Serv Res 1982; 17:5-26.

9. McDonald CJ, Barnett GO. Medical-record systems. In; Shortliffe EH, Perreault LE, eds. Medical Informatics - Computer Applications in Health Care. Reading MA: Addison-Wesley, $1990: 181-218$.

10. Fries JF. Time-oriented patient records and a computer databank, Jama 1972; $222: 1536-42$.

11. Fries JF, McShane DJ. ARAMIS (the American Rheumatism Association Medicall Information System). A prototypical national chronic-disease data bank. West J Med 1986; 145:79:8-804.

12. Blum RL. Discovery, confirmation, and incorporation of causal relationships from a large timeoriented clinical data base: the RX project. Comput Biomed Res 1982; 15:164-87.

13. Fries JF. A data bank for the clinician? [editorial]. N Engl J Med 1976;294:1400-2.

14. Downs SM, Walker MG, Blum RL. Automated summarization of on-line medical records. Proc Medinfo'86 1986:800-804.

15. Kahn MG, Fagan LM, Tu S. Extensions to the time-oriented database model to support temporal reasoning in medical expert systems. Methodls Inf Med 1991; 30:4-14.

16. Kahn MG, Tu S, Fagan LM. TQuery: a context-sensitive temporal query language. Comput Biomed Res 1991; 24:401-19.

17. Das AK, Tu SW, Purcell GP, Musen MA. An extended SQL for temporal data management in clinical decision-support systems. Proc Annu Symp Comput Appl Med Care 1992:128-32.

18. Das AK, Musen MA. A comparison of the temporal expressiveness of three database query methods. Proc Annu Symp Comput Appl Med Care 1995:331-7.

19. McDonald CJ. Protocol-based computer reminders, the quality of care and the non-perfectability of man. N Engl J Med 1976; 295:1351-5.

20. MeDonald CI, Hui SL, Smith DM, et al. Reminders to physicians from an introspective computer medical record. A two-year randomized trial. Ann Intern Med 1984; 100:130-8.

21. McDonald CJ, Hui SL, Tierney WM. Effects of computer reminders for influenza vaccination on morbidity during influenza epidemics. MD Comput 1992;9:304-12.

22. McDonald CJ, Blevins L, Tierney WM, Martin DK. The Regenstrief medical records. MD Comput 1988; 5:34-47.

23. McDonald CJ, Tierney WM, Blevins L. The benifits of automated medical record systems for ambulatory care. In: Orthner HF, Blum BI, eds. Implementing health care information systems. New York: Springer-Verlag, 1988.

24. McDonald $\mathrm{CJ}$, Tierney W/M. Computer-stored medical records. Their future role in medical practice. Jama $1988 ; 259: 3433-40$. 
25. Korpman RA, Lincoln TL. The computer-stored medical record. For whom? [editorial]. Jama $1988: 259: 3454-6$.

26. MeDonald $\mathrm{CJ}$, Terney WM, Owerhage JM, Martin DK. Wilson GA. The Regenstrief Medical Record System: 20 years of experience in hospitals, clinics, and neighborhood health centers. MD Comput 1992; 9:206-17.

27. Whiting OKeefe QE, Whiting A, Henke J. The STOR elinical information system. MD Comput $1988 ; 5: 8-21$.

28. Whiting O"Keefe QE, Simborg DW, Epstein WV, Warger A. A computerized summary medical record system can provide more information than the standard medical record. Jama 1985; $254: 1185-92$.

29. Simborg DW, Whiting OKQE. Evaluation methodology for ambulatory care information systems. Med Care 1982; 20:255-65.

30. Sittig DF, Pace NL, Gardner RM, Beck E, Morris AH. Implementation of a computerized patient advice system using the HELP clinical information system. Comput Bioned Res $1989 ; 22: 474$ 87.

31. Pryor TA. The HELP medical record system. MD Comput $\llbracket 988 ; 5: 22-33$.

32. Huff SM, Pryor TA, Tebbs RD. Pick from thousands: a collaborative processing model for coded data entry. Proc Annu Symp Comput Appl Med Care 1992:104-8.

33. Huff SM, Berthelsen CL, Pryor TA, Dudley AS. Evaluation of an SQL model of the HELP patient database. Proc Annu Symp Comput Appl Med Care 199 1:386-90.

34. Stevens LE, Huff SM, Haug PJ. The development of a wirtual database to provide on-line access to a large archive of clinical data. Proc Annu Symp Comput Appl Med Care 1992:600-4.

35. Huff SM, Haug PJ, Stevens LE, Dupont RC, Pryor TA. HELP the next generation: a new clientserver architecture. Proc Annu Symp Comput Appl Med Care 1994:271-5.

36. Evans RS. The HELP system: a review of clinical applications in infectious diseases and antibiotic use. MD Comput 1991; 8:282-8, 315 .

37. Haug PJ, Gardner RM, Tate KE, et al. Decision support in medicine: examples from the HELP system. Comput Biomed Res 1994; 27:396-418.

38. Gardner RM, Lundsgaarde HP. Evaltuation of user acceptance of a clinical expert system. J Am Med Inform Assoc 1994; 1:428-38.

39. Lam SH. Implementation and evaluation of practice guidelines. Proc Annu Symp Comput Appl Med Care 1993:253-7.

40. Stead WW, Hammond WE, Straube MJ. A chartless record--is it adequate? J Med Syst 1983; $7: 103-9$.

41. Stead WW. Hammond WE. Computer-based medical records: the centerpiece of TMR. MD Comput $1988 ; 5: 48-62$.

42. Hammond WE. TMR - A profile of an electronic patient record, Proc Medinfo'92, 1992. NorthHolland.

43. Hammond. WE, Stead WW. Adopting TMR for physicianhurse use. Proc Annu Symp Comput Appl Med Care 1992:833-837.

44. Barnott $\mathrm{GO}$. The application of computer-based medicall record systems in ambulatory practice. N Ëngl J Med 1984; 310:1643-50.

45. Schoenbaum SC, Barnett GO. Automated ambulatory medical records systems. An orphan technology. Int J Technol Assess Health Care 1992; 8:598-609.

46. Campbell JR, Givner N, Seelig CB, et al. Computerized medical records and clinic function. MD Comput 1989;6:282-7.

47. Chessare $\sqrt{ } \mathrm{B}$, Torok KE. Implementation of COSTAR in an academic group practice of general pediatrics. MD Comput 1993;10:23-7.

48. Dell DV, Tape TO, Campbell JR. Increasing physician acceptance and use of the computerized ambulatory medical record. Proc Annu Symp Comput Appl Med Care 1992:848-852.

49. Safran C, Porter D, Liglitfoot I, et al. ClinQuery: a system for online searching of data in a teaching lospital. Ann Intern Med 1989;111:751-6. 
50. Horowitz GL, Bleich HL. PaperChase: a computer progran to search the medical hiterature. N Engl J Med 1981; 305-924-30.

51. Safran C, Rury C, Rind DM. Taylor WC. A computer-based outpatient medical record for a teaching hospital. MD Comput 1991;8:291-9.

52. Safran C, Rind DM, Davis RB, et al. Effects of a knowledge-based electronic patient record on adherence to practice guidelines. MD Comput 1996:13:55-63.

53. Safran C. Rimd DM, Sands DZ, et al. Development of a knowledge-based electronic patient record MD Comput 1996; 13:46-54.

54. Safran $C$, Rind $D M$, Davis $R B$, et al. Guidelines for management of HIV infection with computer-based patient"s record. Lancet 1995; 346;341-6.

55. Scherrer JR, Baud RH. Hochstrasser D, Ratib O. An integrated hospital information system in Geneva. MD Comput 1990; 7:81-9.

56. Assimacopoullos A, Revillard C, Hertmann F, Borst F, Paschoud N, Scherrer J-R. An lectronic notebook for problem-oriented patient progress notes: testing a concept, Proc Medinfo'89, 1989. North-Holland.

57. Assimacopoulos A, Borgazzi A. An electronic patient record combining free text and coded nomenclature: application to nursing process, Proc Medinfo 92, 1992. North-Holland.

58. Schemer IR, Baud $R$, Borst $\mathbb{E}$. Integrated computerized patient records: a twoyear Geneva experience. Int J Biomed Comput 1996:42:123-128.

59. Scherrer JR, Revillard C, Borst F, Berthoud M, Lovis C. Medical office automation integrated into the distributed architecture of a hospital information system. Methods Inf Med 1994:33:1749.

60. Borsit F. [personal communication], 1996.

61. Bolens M, Borst F, Scherrer JR. Organizing the clinical data in the medical record. MD Comput 1992: 9:149-55.

62. Baud RH, Rassinoux AM, Scherrer JR. Natural language processing and semantical representation of medical texts. Methods Inf Med 1992; 31:117-25.

63. Rassinoux AM, Wagner JC, Lovis C, Band RH, Rector A, Scherrer JR. Analysis of medical texts based on a sound medical model. Proe Annu Symp Comput Appl Med Care 1995:27-31.

64. Rector AL, Solomon WS, Nowlan WA, et al. A terminology server for medical language and medical information systems. Methods Inf Med 1995; 34:147-157.

65. Kent DL, Shortliffe EH, Carlson RW, Bischoff MB, Jacobs CD. Improvements in data collection through physician use of a computer-based chemotherapy treatment consultant. $\mathrm{J}$ Clin Oncol $1985 ; 3: 1409-17$.

66. Musen MA. Wieckert KE. Miller ET, et al. Development of a controlled medical vocabulary: knowledge acquisition and knowledge representation. Methods Inf Med 1995; 34:85-95.

67. Rothwell DI. SNOMED-based knowledge representation. Methods Inf Med 1995; 34:209-157.

68. Campbell KE. Musen MA. Representation of clinical data using SNOMED III and conceptual graphs. Proc Annu Symp Comput Appl Med Care 1992:354.8.

69. Campbell KE, Das AK, Musen MA. A logical foundation for representation of clinical data. I Am Med Inform Assoc 1994; 1:218-32.

70. Sowa JF. Conceptual structures. Reading MA: Addision Wesley, 1984.

71. Johnson K, Poon A, Shiffman S, Lin R, Fagan L. A history-taking system that uses continuous speech recognition. Proc Annu Symp Comput Appl Med Care 1992.757-61.

72. Poon AD, Fagan LM, Shortliffe EH. The PEN-Ivory project: Exploring user-interface design for the selection of items from large controlled wocabularies in medicine. J Am Med Inform Assoc $1996 ; 3: 168-183$.

73. Campbell KE. Wieckert K, Fagan LM, Musen M.A. A computer-based tool for generation of progress notes. Proc Annu Symp Comput Appl Med Care 1993:284-8.

74. Lin $\mathbb{R}$, Lenert $L$, Middleton $B$, Shiffman $S$. A free-text processing system to caplure physical findings: Canonical Phrase Identification System (CAPIS). Proc Annu Symp Comput Appl Med Care 1991:843-7. 
75. Rector AL, Nowlan WA, Kay $\$$. Foundations for an electronic medical record. Methods Inf Med $1991 ; 30: 179-86$.

76. Rector AL; Nowan WA, Kay S, Goble CA. Howkins TJ. A framework for modelling the dectronic medical record. Methods Inf Med 1993; 32:109-19.

77. 粈ector AL. Marking up is not enough [editorial]. Methods Inf Med 1993; 32:272-3.

78. Nowlan WA, Rector AL, Kay $\$$. Horan B, Wilson A. A patient care workstation based on user centred design and a formal theory of medical terminology: PEN\&PAD and the SMK formalism. Proce Annu Symp Comput Appl Med Care 1991:855-7.

79. Heallifield HA, Hardiker $\mathrm{N}$, Kirby $\mathbb{J}$, Tallis $\mathrm{R}$, Gonsalkarale $M$. The PEN \& PAD medical record model; development of a nursing record for hospital-based care of the elderly. Methods Inf Med $1994: 33: 464-72$.

80. Heathlife $\mathrm{HA}$, Kirby J, Hardiker NR. Data entry in computer-based care planning. Comput Methods Programs Biomed 1995; 48:103-107.

81. Rector AL, Glowinski AJ, Nowlan WA, Rosso-Mori A. Medical-concepts models and medical records: an approach based on GALEN and PEN\&PAD. J Am Med Inform Assoc 1995; 2:19-35.

82. Kay $S$, Purves IN. Medical records and other stories: a narratological framework. Methods Inf Med 1996; 35:72-87.

83. Timmers $T$, van Mulligen EM, van den Heuvel $F$. Integrating clincial databases in a medical workstation using knowlegde-based modeling, Proc Medinfo" 92 , 1992. North-Holland.

84. van Ginneken AM, Stan $\mathrm{H}$, Moorman PW. A multi-strategy approach for medical records of specialists, Proc Medinfo'95, 1995. North-Holland.

85. van Gimneken AM, Stam H. Can one patient record accommodate the diversity of specialized care? Proc Annu Symp Comput Appl Med Care 1995:406 10.

86. Moorman PW, van Ginneken AM, van der Lei J, wan Bemmel JH. A model for structured data entry based on explicit descriptional knowledge. Methods Inf Med 1994; 33:454-63.

87. Timmers T, Pierik F, Steenbergen M, et al. ARIS: integrating multi-source data for research in andrology. Proc Annu Symp Comput Appl Med Care 1995:445-8.

88. Moorman PW, van Ginneken AM, Siersema PD, van der Lei J, van Bemmel JH. Evaluation of reporting based on descriptional knowledge. J Am Med Inform Assoc 1995; 2:365-73.

89. Cimino JJ, Clayton PD. Coping with changing controlled vocabularies. Proc Annu Symp Comput Appl Med Care 1994:135-9.

90. Cimino J, Socratous SA, Clayton PD. Internet as clinical information system: application development using the World Wide Web. J Am Med Inform Assoc 1995; 2:273-84.

91. Barrows RC, Jr., Johnson SB. A data model that captures clinical reasoning about patient problems. Proc Annu Symp Comput Appl Med Care 1995:402-5.

92. Hincoln TL, Essin DJ. A polemic about hypotheses: a missing perspective in medical informatics Ieditorial]. Methods Inf Med 1992; 3:1:2.

93. Lincoln TL, Essin DI. Ware WH. The electronic medical record: a challenge for computer science to develop clinically and socially retevant computer systems to coordinate information for patient care and analysis. The Information Society 1992; 9:157-188.

94. Fssin DJ. Intelligent processing of loosely structured documents as a strategy for organizing electronic health care records. Methods Inf Med 1993: 32:265-8.

95. Lincoln TL, Essin DJ. A document processing architecture for electronic medical records, Proc Medinfo 95,1995 . North-Holland.

96. Essin DJ, Lincoln TL. Implementing a low-cost computer-based patient record: a controlled vocabulary reduces data base design complexity. Proc Annu Symp Comput Appl Med Care $1995: 431.5$.

97. Trace D, Naeymi Rad F, Haines D, et al. Intelligent Medical Record--entry (IMR-E). J Med Syst $1993 ; 17: 139-51$

98. Nygren E, Henriksson P. Reading the medical record. I. Analysis of physicians' ways of reading the medical record. Comput Meihods Programs Biomed 1992; 39: 1-12. 
99. Nygren $E$, Johnson M, Henriksson P. Reading the medical record. II. Design of a human computer interface for basic reading of computerized medical records. Comput Methods. Programs Biomed 1992; 39:13-25.

100. Sager N, Friedman C, Lyman MS, et al. Medical language processing: conputer management of narrative data. Reading MS: Addison-Wesley, 1987.

101. Sager $N$, Lyman M, Nhan NT, et al. Clnical knowledge bases from natural language patient documents, Proc Medinfo"92, 1992. North-Holland.

102. Sager N, Lyman M, Tick LJ, Ngồ TN, Bucknall CE. Natural language processing of asthma discharge summaries for the monitoring of patient care. Proc Annu Symp Comput Appl Med Care 1993:265-268.

103. Bell DS, Greenes RA, Doubilet P. Forn-based clinical input from a structured vocabulary: Initial application in ultrasound reporting. Proc Annu Symp Comput Appl Med Care 1991:798790.

104. Bernauer $\mathrm{J}$. Conceptual graphs as an operational model for descriptive fundings. Proc Annu Symp Comput Appl Med Care 1991:214-8.

105. Gonveia Oliveira A, Raposo VD, Azevedo AP, et al. SISCOPE: a multiuser information system for gastrointestinal endoscopy. Endoscopy 1991; 23:272-7.

106. Gonveia Oliveira A, Raposo VD, Salgado NC, Almeidla I., Nobre Leitao C, de Melo FG. Longitudinal comparative study on the influence of computers on reporting of clinical data. Endoscopy 1991; 23:334-7.

107. Kuhn $K$, Gaus W, Wechsler JG, et al. Structured reporting of medical findings: evaltuation of a system in gastroenterology. Methods Inf Med $1992 ; 31: 268-74$.

108. Barnet: GO, Jenders RA, Chueh HC. The computer-based clinical record-where do we stand? [editorial]. Ann Intern Med 1993; 119:1046-8.

109. Hammond WE. The role of standards in creating a health information infrastructure. Int $J$ Biomed Comput 1994; 34:29-44.

110. Blois MS. Information and medicine - the nature of medical dscriptions. Berkeley, CA: Unversity of California Press, 1984.

111. Chute CG, Cohn SP, Campbell KE, et al. The content coverage of clinical classifications. J Am Med Inform Assoc 1996; 3:224-233.

112. Gregory J, Mattison JE, Linde C. Naming notes: Transitions from free text to structured entry. Methods Inf Med 1995:57-67.

113. Poon AD, Fagan LM. PEN-Ivory: the design and evaluation of a pen-based computer system for structured data entry. Proc Annu Symp Comput Appl Med Care 1994:447-51. 



\title{
4. How to approach the structuring of the medical record? - Towards a multiple-view searching structure
}

\author{
Huibert Tange \\ Department of Medical Informatics, University of Maastricht
}

The development and dissemination of electronic medical records is impeded because of several reasons, among which the unruliness of narrative data. In this chapter, we explain how the medical record can be structured to make it searchable from different perspectives, without interfering with the format of medical data, hence without the need to avoid the use of free text in medical narratives. We analyse the organising principles behind the source-oriented, time-oriented, and problemmoriented medical record, resulting in a description of four views on medical data: a time view, source view, problem view, and process view. Some of these views have alreadly been used in existing electronic medical-record systems, but not for free-text medical narratives. We combine these four views in one data model for an electronic medical-record system. We believe that this idea of multiple views should be the basis of any electronic medical-record system. However, some practical issues related to the design of such a system must be addressed yet: the granularity of the medical-record structure, and the design of the user interface.

Published as:

Tange HJ. How to approach the structuring of the medical record? Towards a model for flexible access to free-text medical data. Int J Biomed Comput 1996;42;27-34. 
Chapler 4

56 


\subsection{Introduction}

Who wants to use the medical record as an effective, multi-authored, communication tool will need more functionality than a paper medical record can offer. The amount of clinical data is growing, Jeading to thicker medical records and making the retrieval of information more difficult. These and many other arguments have been forwarded to indicate that the medical record should be computerised. An electronic medical record is better legible, can provide remote and simultaneous access, can present its content in different ways, and can be extended with validity checks, reminders, alerts, and other decision-support tools. ${ }^{2}$ Yet, their dissemination has been slow. Various reasons have been suggested for this lack of progress, not only technical arguments but also others such as the unruliness of narrative data, the defensive attitude of physicians towards the use of computers, and unsolved problems with respect to privacy. ${ }^{3}$

With medical narratives we mean the original physician-gathered data recorded in medical history, physical examination, and progress notes. Most narrative data result from qualitative observations. Their unruliness reflects the lack of consensus among professionals about their inner structure as well as the format in which these data are stored. The structure of the medical record - the way medical data are grouped together - is expressed in the way it is divided into indexable sections. Via such an index, the physician can focus on the relevant parts of the medical record. A good structure makes the medical record better searchable. The structure of the medical record should not be confused with the format of medical data, which is a matter of understandability of their content. Traditionally, the format of medical narrative data is free text. Since the appearance of the first electronic medical-record systems the urge has been felt to standardise and code medical data, including narratives. Computers excel in the manipulation of structured data, but are less useful for the disclosure of free-text information. Especially for management and research purposes a standardised medical-record content is superior to free text. However, coding free text has a cost: an inevitable loss of nuanced information. ${ }^{4}$ Several efforts to develop and improve controlled vocabularies in order to minimise the loss of information are ongoing, but most of them are far from a complete coverage. ${ }^{5}$

In this article, we elaborate on the structure of the medical record as a means to improve the retrieval of information. We leave the medical data in their original format, which is free text for narrative data. The structure of the medical record in generall, and of medical narratives in particular, has been subject of discourse since about 25 years, when the problem-oriented and time-oriented medical record were proposed as alternative structures for the, traditional, source-oriented medical tecord. In 4.2 , we analyse the organising principles behind the source-oriented, problemoriented, and time-oriented medical-record structure, as described in the literature. In 4.3, we combine these principles as alternative views in one single data model for electronic medical-record systems. 


\subsection{Different medical-record structures}

The structure of the medical record has been subject of discourse for some decades. The traditional medical-record structure is source-oriented, and has been used in hospitals since the beginning of this century. Around 1970, two alternative structures were introduced, the problem-oriented and the-omiented medical record. Although their advantages have been emphasised by many, they did not surpass the traditional medical record in daily practice. This was a reason for us to analyse and isolate the useful elements from the different medical-record structures.

\subsubsection{The source-oriented medical record}

The traditional medical-record structure originates from the beginning of this century, the time that additional diagnostic procedures, like radiology and pathology, became important and the hospital became the corner stone of medical care. Initially, each department in the hospital kept its own medical record (a practice that continued, in some hospitals, until the seventies). However, already in 1907 it was for the first time recommended to put all deparmental records together in one clinical record per patient." This single medical record was divided into sections, according to the departments where the information was generated. The source-oriented medical record was born.

In the source-oriented medical record, medical data are grouped type by type. The types of data are organised in a hierarchical way, according to different criteria. The primary criterion is the source of medical data, hence the name source-oriented. Further criteria may vary. For example, to locate the result of a blood-sodium laboratory test, one must first select the source of information (laboratory), and subsequently the type of test (clinical chemistry), its specimen (blood), and its substrate (sodium). Together, these criteria form a hierarchy of medical-data types. Each child type is a further specialisation of its parent type. On top of the hierarchy is a, virtual, ancestor medical-dlata type. We prevail the term source-oriented for indicating this entire hierarchical organisation, because it is widely known as such.

The source organisation is universal for every patient. It makes the location of data in the medical record predictable, regardless of the case. Also medical narratives can be structured according to this source-oriented principle. In fact they have a certain pattern (only hidden in the text): the sequence of criteria used reflects the physician's work-up while gathering data. For example, to find clinical data with respect to palpation of" the liver, one can browse through the text following the criteria: source (physician), technique (physical examination), anatomic localisation (abdomen), technique (palpation), and again anatomic localisation (liver). For progress notes, however, the traditional, source-oriented medical record does not provide an inner structure. This part of the medical record is just a repository of medical data of different types, reported in chronological order.

\subsubsection{The time-oriented medical record}

The time-oriented medical record, introduced by Fries in 1971, has been developed in an environment of chronically ill patients, mainly from outpatient settings. Its primary 
purpose was to serve as a national research data bank with longitudinal clinical data of chronic rheumatologic diseases, ${ }^{7,8}$ but it has also proved to facilitate the retrieval of medical data during the patient encounter itself."

Time is a universal organising principle, which can be applied to any nedical record containing time-stamped (time-indexed) data. To exploit this principle as much as possible, Fries multiplied the number of time-indexable data by cuting the medicalhistory and physical-examination section into many detailed paragraphs and by registering such data, also in the period after intake, within these paragraphs. As a consequence, progress notes were stripped and contained merely information about medical judgement, hence Fries renamed this section into medical assessment.

Typical for the time-oriented medical record is the two-dimensional presentation of medical data in a matrix, allowing instant access to medical data from two perspectives: type of data, and time of registration. One column in this matrix represents a snapshot of the present state at a certain moment, one row represents the progress of a certain type of medical data, e.g., one separate kind of observation. Thus, in the time-oriented medical record, present state and progress notes are represented as two dimensions of the same data instead of as separate sections in the medical record. Some authors stress, that a single notion of time is ambiguous and should be further specified as moment of initiation, moment of registration, and the moment the insight was gained. Nevertheless, for each time-stamp the idea of time as a dimension of medical data remains valid, whether it be the time of initiation, registration, or insight gained.

Paper medical records have the physical limitation that a cell of a two-dimensional matrix can only contain short descriptions. Although this is enough for numbers and codes, it is usually not sufficient for the storage of full-length free text. In Fries' original time-oriented record all narrative data were coded. ${ }^{7}$ on the other hand, computer data can be stored independent from the way they are presented on the screen. This makes it possible to maintain a matrix presentation in which one cell can give access to a large amount of free text.

\subsubsection{The problem-oriented medical record}

The problem-oriented medical record was developed by weed in the late sixties to encourage physicians and medical students not to focus on the determination and treatment of one single diagnosis and to also consider other than medical diagnoses." Patients suffer from several, more or less urgent, problems, each of which deserves attention from the physician. This multiplicity of problems makes the physicians' work different from the work of other scientists'. Weed assumed that physicians are not sufficiently prepared to identify the patient's problems and to organise these problems for solution. He developed the problem-oriented medical record to support them in this task. Evidently, his ideas about medical-record keeping have a strong educational emphasis. ${ }^{12}$ He describes the generation of a medical record as follows. As the first step, a complete initial database of intake data is created, organised in the traditional, source-oriented way. Next, a comprehensive problem list is formulated, together with an initial plan. After intake, progress notes are maintained of the most urgent (active) problems, each in a separate section. Within such a section, medical data are ordered in 
the sequence of Subjective observations, Objective observations, Assessment, and Plian (SOAP). This SOAP-principle is the reflection of systematic medical care, which is based on the assumption that the physician's reasoning follows a systematic pattern.

Thus, in the problem-oriented medical record, progress notes are structured according to two different organising principles. The first principle is the problem organisation: each medical problem is linked with a list of medical-data types that can be relevant for the problem. Simllar to the source organisation, the problem organisation (which is also a grouping of data types) can be presented in a twodimensional type-by-time flowsheet. An example of such a problem-oriented flowsheet is a special form designed for monitoring diabetic patients. Such a form includes a selection of medical-data types, such as blood-glucose level, insulin medication, etc. Different from the source organisation, on the other hand, is the absence of a hierarchy and the existence presence of an overlap of medical-data types between different problem organisations. The second organising principle of progress notes in the problem-oriented medical record is the process organisation: each medical problem is linked with a list of medical data that are certainly relevant for the problem. Weed implemented this process organisation in the sequence of data (SOAP) within a progress note, hence reflecting the physician's way of thinking. The process organisation is useful for the reconstruction of medical practice, which facilitates the co-ordination of care between disciplines and may serve as an illustration for educational purposes.

In its practical implementation, the maintenance of a full-fledged problem-oriented medical record is very laborious. Observations relevant to more than one problem must be entered more than once. To avoid laborious documentation, it is also possible to adopt onlly the process organisation. ${ }^{13}$ But also from a methodological point of view, the (dis)advantages of the problem-oriented medical record have been subject of fervent discussions. . $^{-16}$ Feinstein criticised the problem-oriented medical record heavily by objecting that a partitioning of progress notes into problems impedes the association of data across problems, e.g., for making up real medical diagnoses. Furthermore, the absence of standard definitions would make the meaning of problems unclear, 16

\subsection{Integration of organising principles}

The three medical-record structures that were described differ mainly in the way how medical narratives are structured. Four organising principles were elucidated: (1) the source organisation in which medical data are grouped type by type in a hierarchical way, (2) the problem organisation in which medical data are grouped type by type according to their relevance to a medical problem, (3) the time organisation in which medical data are ordered according to the time of their recording, and (4) the process organisation in which medical data are related to one another according to the role they have played in the delivery of medical practice. Each organising principle has its strengths and weaknesses. The source organisation and time organisation provide a generally applicable searching structure which, by definition, cannot be tailored to the 


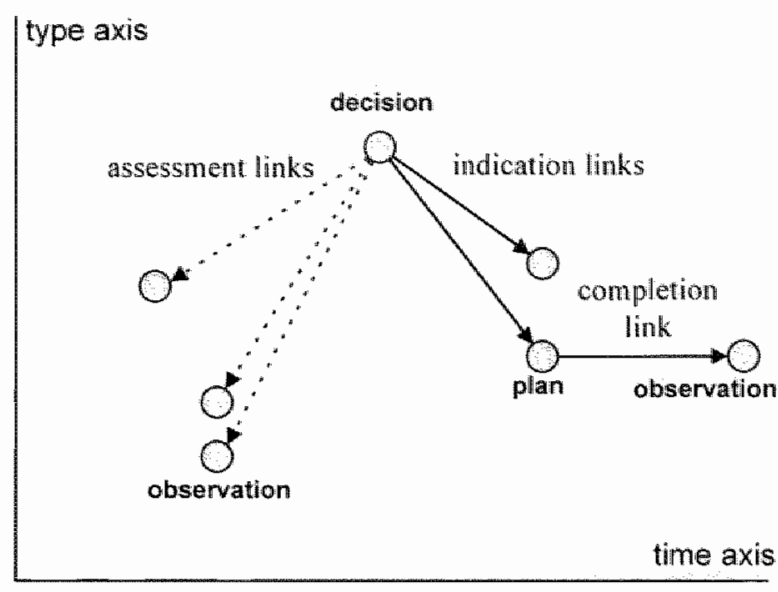

Figure 4- 1 :

Vector representation of the process organisation in the two-dimensional searching space.

question under concern. The problem organisation is specific for patients with similar medical problems, but looses its value outside this context. The process organisation, finally, is unique for each single decision a physician takes. This organisation provides a reconstruction of the medical-care process in general and of the rationale behind medical decisions in particular. It is useful in the setting of shared medical care, and also for purposes of medical education and medical audit.

It is evident, that the different organising principles described are complementary to one another. To take advantage of them all when searching, one needs to use a medical record in which all four organising principles are integrated. In this paragraph, we explain how this can be achieved in an electronic medical-record system.

\subsection{Definition of a two-dimensional searching space}

Each medical data can be identified by two co-ordinates: type of medical data and time of registration. (Time of registration can be replaced by time of initiation or insight if wished. $)^{10}$ Together, these co-ordinates form a two-dimensional searching space (Figure 4-1). The type dimension can be organised source-oriented or problemoriented. The organisation of the time dimension is self-evident. The process organisation, on the other hand, is not a means for searching a single medical data, but for establishing a meaningful relation between two or more medical data that each individually can be identified by two co-ordinates in the two-dimensional searching space. Thus, a process link can be represented as a vector between medical data in this two-dimensional searching space. From Weed's SOAP-structure, three kinds of process links can be deduced:

1. Assessment links between a decision (soAp) and the observations on which this decision is based (SOap)

2. Indication links between a decision ( $\mathrm{SOAp}$ ) and the plans for further action (soaP) that are indicated 


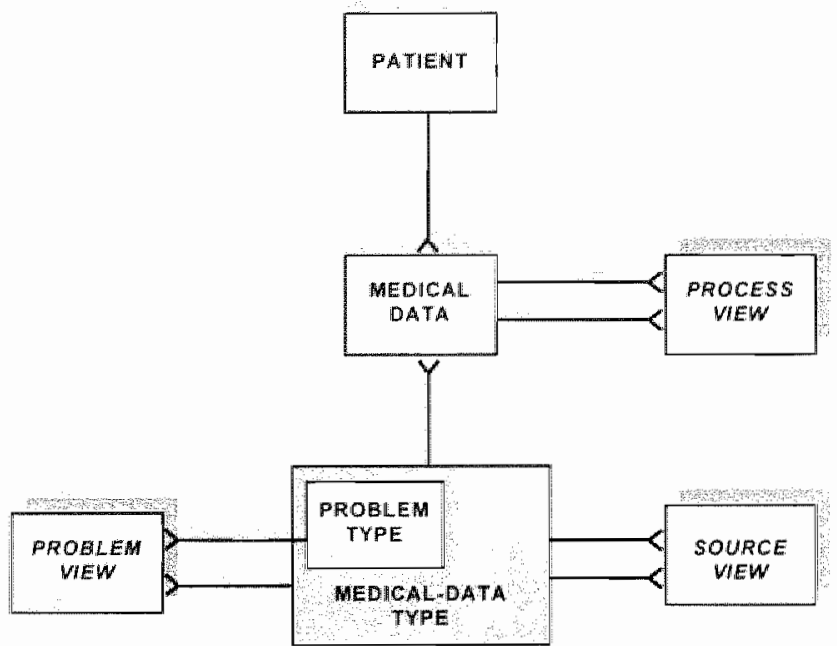

Figure 4-2:

Conceptual data model of the struclure of the medical record. The different views of the searching structure are shadowed. The terminology base is in gray.

Symbols and lines are according to the entity-relationship-diagramming technique.

3. Completion links between planned activities (soaP) and the corresponding results (SOap).

\subsubsection{A multiple-view data model}

The implementation of the different organising principles is a matter of storing the specifications of each organisation (except time) in the database of the medical record. The source organisation is stable, so it has to be defined only once, the problem organisation should be customisable to the physician's way of working and the process organisation must be explicitly specified during medical practice itself. To facilitate the maintenance of these structures, we propose to store each specification in a separate table of the database. In Figure 4-2, the relevant part of the conceptual data model is presented as an entity-relationship diagram.

Central in this model is the table MEDICAL-DATA TYPE, which contains terms for observations, procedures, medications, treatments, laboratory tests, etc. Also the names of, predelined, medical problems are stored in this table. We represent these problem names as a specific sub-entity PROBLEM TYPE. In the table MEDICAL DATA, clinical information about specific patients can be stored. Per default, this information is stored as free text, but also storage in other formats is possible (codes, numbers, pictures). The table PATIENT contains idlentifying and demographic information about the patients.

The searching structure of the medical record is specified in three tables:

- The table SOURCE VIEW specifies the source-oriented structure of the medical record in terms of parent-child links between medical-data types. All records together form a hierarchic structure. For example, palpation of the abdomen is specified as a child of physical examination, and blood-sodium test is a child of 


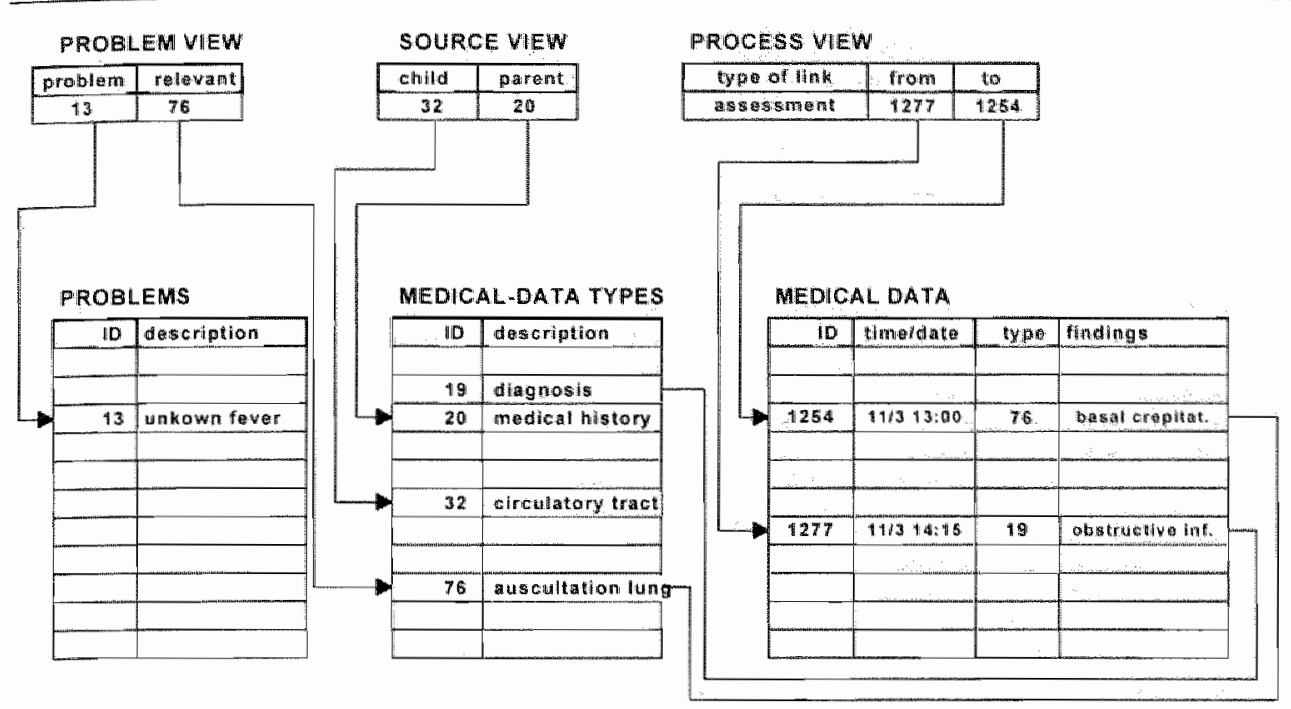

Figure 4-3

Example of some specifications of views (represented in a technical data model).

clinical-chemistry test, and a grand child of laboratory test. Physical examination and laboratory test, in turn, are children of medical record, the generic ancestor of all other medical-data types and located on top of the hierarchy. Each row in this table links a parent medical-data type with a chilld medical-data type, both specified in the table MEDICAL-DATA TYPE.

- The table PROBLEM VIEW specifies the links between a type of problem and the different types of medical data that are considered relevant for this problem type. Each row in this table links a problem type with a medical-data type, both specified in the table MEDICAL-DATA TYYPE..

- In the table PROCESS VIEW, the process of medical care can be recorded by connecting different medical data from the table MEDICAL DATA with one another. Each row in this table also contains a specification of the kind of association (assessment, indication, or completion).

In Figure 4-3, we demonstrate how the different views must be interpreted. From the source view, the following information can be read: Circulatory ract is a child type of medical history. The problem view specifies: Auscultation of the lung is an item relevant to the problem unknown fever. The process view, finally, expresses the following: On March 1. Ith, 2:15 PM, (with respect to patient $X$, not visible in the model) the diagnosis obstructive infection of the lung was assessed by the finding basal crepitations observed on March 11/h, 1:00 PM, as the result of auscultation of the lung.

\subsection{Discussion}

The problem of structuring the medical record has been point of discussion for a long time. We believe this discussion has been unnecessarily complicated by several. 
factors. First, most authors do not clearly distinguish between the structure of the medical record and the format of medical data. Structuring the medical record makes it better searchable, while formatting medical data makes its content unambiguously understandable. Second, the discussion about the structure of the medical record has always been dominated by adepts and opponents of the problem-oriented medical record. The (dis-)advantages of the source-oriented and time-oriented medical record were not much taken into account. This one-sided emphasis did not contribute to an open and objective comparison of principles. Moreover, the problem view and process wew were not treated as two different organising principles, hence a proper understanding and assessment of their respective values has never been obtained.

In this chapter, we hope to have described the problem of structuring the medical record in a clear-cut way. Al least, we have demarcated this subject from the problem of formatting medicall data. We have taken all known medical-record structures into account and have presented their organising principles as views that are complementary to one another.

The combination of views as described in this chapter has already been implemented in several electronic medical-record systems, be it not explicitly and only for coded data. The familiar tow-dimensional presentation of Iaboratory results in the traditional, paper-based, medical record is an evident example of the combination of source view and time view, a so-called source-oriented flowsheet. The combination of problem view and time view is known as problem oriented flowsheets and was already described in different classical electronic medical-record systems." The time-oriented medical record is in fact one big source-oriented flowsheet, comprising the whole medical record, including (coded) medical narratives. ${ }^{\$ 17}$ The lime view as such was subject of some recent studies concerning extensions to SQL (the standard query language for relational database-management systems) that should facilitate temporal reasoning. 18.19

We did not find evidence, however, of successful implementations of the process view in existing electronic medical-record systems. Weed's own PROMIS-system was the first attempt to computerise the problem-oriented medical record in its totality, hence including the process view. ${ }^{20}$ The PROMIS-system did not survive, which was attributed by others, to its "dogmatic nature". "For example, the physician was urged through eight steps when generating a plan for further action. Evidently, the use of PROMIS in general, and probably the urge of maintaining a scrupulous process view in particular, demanded too much documentation discipline from the physician. The growing need for getting insight in the delivery of medical practice has resulted in a renewed interest in the process view. The principle of linking medical data to one another to get insight in the process of medical care was recently described by several authors. Van Ginneken et al. called these links, confusingly, "problem-oriented links". 21 Barrows et al. included these links into the data model of a medical record but admitted that they encountered "logistic problems with the interface" when trying to implement these links. ${ }^{22}$ Apparently, despite the advances of information technology since PROMIS, it still seems difficult to design an appropriate user-interface for the 
process view. We did not concentrate on this problem, but it certainly needs further investigation.

Another unsolved issue is the question how detailed the narratives parts of the medical record should be partitioned when defining nedical-data types. Which granularity of paragraphs will provide the most profitable combination of views for the searching structure? For research purposes medical data cannot be structured enough. because the more detail in this structure, the more precise medical data can be located. On the other hand, too much detail leads to unnecessary fragmentation of information. which may tears medical data out of their clinical context. This may be disadvantageous when using the medical record to retrieve information for direct medical practice. In Chapter 7, we report a study concerning the effect of granularity of medical narratives on the ease of information retrieval for this purpose.

Much of the functionality of an electronic medical-record systen relies on its underlying data model. However, the last two issues discussed above illustrate the fact that a proper data model in itself is not a guarantee for a good information system. There are more aspects to be dealt with before one can speak of a toral concept lor an electronic medical-record system. We hope to have contributed to a better understanding of at least one of them.

\subsection{Conclusion}

After decades of debate about how to structure the medical record, the time has come to combine the best elements of the different approaches. A clear distinction between source view, time view, problem view, and process view is essential when designing the conceptual data model for any electronic medical-recond application. Existing medical-record systems often combine two organising principles into source oriented flowsheets and problem-oriented flowsheets, respectively, however for numeric and coded data only. We believe that the format of medical data does not put restrictions to the structure of the medical record. Hence, we presented a muliple-view data model that is also applicable to free-text medical narratives. Before this data model can be implemented in an operational medical-record system, some other problems must be solved, such as the question of the desired granularity of medical narratives and the question how to design a user-friendly interface for maintaining the process vicw.

\subsection{References}

1. Tange HJ. How to approach the structuring of the medical record? Towards a model for flexible access to free-text medical data. Int J Biomed Comput 1996; 42:27-34.

2. MoDonald CJ, Barneti GO. Medical-record systems. In: Shortlife EH, Perreault LE, eds. Medical Informatics - Computer Applications in Health Care. Reading MA: Addison-Wesley, 1990:181-218.

3. Dick RS, Stein $\mathbb{E B}$. The computer-based patient record - an essential technology for health care. Washington DC: National Academy Press, 1991.

4. Blois MS. Information and medicine - the nature of medical dscriptions. Berkeley, CA: Unversity of California Press, 1984. 
5. Chue $\mathrm{CG}$, Cohn $\mathrm{SP}$, Campbell $\mathrm{KE}$, et al. The content coverage of clinical classifications. I Am Med Inform Assoc 1996; 3:224 233.

6. Reiser S3. The clinical record in medicine. Part 2: Reforming content and purpose. Ann Intern Med 1991:114980-5.

7. Fries JF. Time-oriented patient records and a computer databank. Jama 1972; 222:1536-42.

8. Fries JF, MeShane DJ. ARAMIS (the American Rheumatism Association Medical Information System). A prototypical national chronic-disease data bank. West J Med 1986; 145:798-804.

9. Fries JF. Alternatives in medical record formats. Med Care 1974, 12:871-81.

10. van Ginneken AM. The structure of data in medical records. In: van Bemmel JH, McCray AT, eds. The Computer-based Patient Record. Stukgart: Schattauer, 1995:61-70.

11. Weed LL. Medical records that guide and reach. N Engl J Med 1968; 278:593-600,652-657.

12. Weed LL. Medical records, medical education, and patient care. Chicago: Year Book Medical Publishers, lne., 1969.

13. de Vries Robbé PF. Useful elements of P.O.M.R - a report of experience. Medical Record 1975; $16: 13-17$.

14. Coldfinger SE. The problem-ariented record: a critique from a believer. $N$ Engl J Med 1973; 288:606-8.

15. Donnelly WJ, Hines E, Brauner DJ, Why SOAP is bad for the medical record. Arch Intern Med $1992 ; 152: 481-84$

16. Feinstein AR. The problems of the "Problem-Oriented Medical Record". Ann Intern Med 1973; $78: 751-762$.

17. Bolens $M_{*}$ Borst F, Scherrer JR. Organizing the clinical data in the medical record. MD Comput 1992: 9:149-55.

18. Kahn MG, Fagan LM, Tu S. Extensions to the time-oriented database modell to stipport temporal reasoning in medical expert systems. Methods Inf Med 1991; 30:4-14.

19. Das AK, Tu SW, Purcell GP, Musen MA. An extended SQL for temporal data management in clinical decision-support systems. Proc Annu Symp Comput Appl Med Care 1992:128-32.

20. PROMIS-Laboratory. Representation of medical knowledge and PROMIS. Proc Annu Symp Comput Appl Med Care 1978:368-400.

21. van Ginneken AM, Stam H, Moorman PW. A multi-strategy approach for medical records of specialists, Proc Medinfo'95, 1995. North-Holland.

22. Barrows RC, Jr, Johnson SB. A data model that captures clinical reasoning about patient problems. Proc Annu Symp Comput Appl Med Care 1995:402-5. 


\section{A conversation model of medical practice - How to get a process view on shared medical care}

H.J. Tange, J.L.G. Dietz ${ }^{b}$, P.F. de Vries Robbée

${ }^{a}$ Department of Medical Informatics, Maastricht University

b Department of Information Systems, Faculty of Technical Mathematics and Informatics, University of Delft

'Department of Medical Informatics, Epidemiology, and Statistics, University of Nijmegen.

Among different parties in health care there is a growing need for information about the process of medical care. A medical record that provides a process view would be welcome, but is difficult to specify, especially in the setting of shared medical care. We first discuss some existing models reflecting the process of medical practice. We then present a model of medical practice in which current theories of systematic medical practice are integrated and that is also applicable to shared care. In this conversation model, a medical data is the result of a conversation between two actors that may be personified by different persons or by one and the same person. We subsequently present a data model of the process view, based on this conversation model. In the discussion, we argue that the conversation model of medical practice is generally applicable and robust against organisational changes, and that it provides an understanding of medical practice that may also serwe other purposes such as medical education, medical-decision support, information planning, and business-process redesign.

To be submitted 
Choprew 5 


\subsection{Introduction}

At present, the organisation of health care is changing rapidly. Adwances in medical knowledge and medical technology have transformed health care to a complex collaborative effort rather than an individual task. ${ }^{1}$ The boundaries of competence between primary care, specialties, and subspecialties are shifting. ' Hospital management, governmental authorities and health-insurance companies show a growing interest in the delivery of medical practice. ${ }^{3}$ From these sides - at least in The Netherlands - there is a growing pressure towards a more efficient and effective health care. ${ }^{4}$ These and other developments, both inside and outside the medical profession, stimulate the computerisation of the medical record. In particular, the need to adopt a medical record that also reflects the process of medical practice is acknowledged widely. ${ }^{3}$ However, such a reflection or process view on patient medical data is difficult to specify. We did not find evidence of operational electronic medical-record systems that fully support this view.

The best known process view on the medical record is the partitioning of progress notes into subjective and objective observations, assessment, and plan, the so-called SOAP-structure. This SOAP-structure is one of the aspects of Weed"s problemoriented medical record. ${ }^{5}$ However, for a full specification of the process of medical practice, the SOAP-structure has some shortcomings. In this chapter, we address these shortcomings and explain the complementary value of other approaches of systematic medical practice, as described by Elstein en Rector. ${ }^{6.7}$ We propose an integration of these approaches into a conversation model of collaborative, shared, medical practice in which any information about patients is conceived as being generated by a conversation, either between persons or within one individual. We also describe how this conversation model can be used to accommodate the data model of an electronic medical-record system with a process view that provides insight in how medical care is delivered.

\subsection{Models of systematic medical practice}

\subsubsection{Weed's problem-driven model}

In the late sixties, Lawrence $\mathrm{L}$. Weed introduced the problem-oriented medical record. ${ }^{5.8}$ It was received as a revolutionary approach to medical-record keeping, because it was the first medical record meant to support systematic medical practice. The problem-oriented medical record structure is based on three principles:

1. Collection of a complete initial database - A comprehensive record of chief complaint, patient profile, past history, present illness and systerns review, physical examination and a set of base-line procedures (laboratory, X-ray) should be collected for each case. These base-line diagnostic procedures should be routine rather than case-specific. 
2. Indexing progress notes to problems - Weed criticised traditional medical practice about the fact that physicians are prone to think and work diagnosis-directed, reducing the patient to an object of care. He proposed to regard each patient as having a complex of physical, psychic and social characteristics (problems) that must be recognised and documented in a problem list. Medical practice should be problem-driven, which can be expressed in the medical record by indexing followup information to problems from the problem list.

3. Reflecting the process of medical practice in progress notes - New findings (observations) lead to new insights, which in turn will lead to new plans, followed by new findings, etc. This iterative work-up should be expressed in the SOAPstructure of progress reports: subjective findings, objective findings, assessment, and plan.

Weed's ideas were normative and put emphasis on the education of students and physicians. Weed claimed that the problem-oriented medical record results in a better understanding of medical practice. ${ }^{9}$ Whether the use of the problem-oriented medical record would also lead to better quality of care, has always been subject of discussion. Goldfinger opposed, that creating a complete initial database would be an illusion, and that the separation between initial and follow-up observations would be undesirable. ${ }^{10}$ Feinstein criticised, that problems are often badly defined, hence grouping medical data to problems would obscure rather elucidate the physician's insight." Moreover, such compartmentalisation of medical data would have the potential of impeding "synthetic thinking". (i.e., searching a common cause for several problems). . $^{10.11}$ According to Donnelly et al., Weed's distinction between "subjective" and "objective" observations is highly artificial and should be replaced by a distinction between "history" and "observations": HOAP instead of SOAP. ${ }^{22}$ More generally, Weed's emphasis on the format of medical documentation would divert the physician's attention from the real problem: the accuracy of medical data. ${ }^{13}$ Nonetheless, the problem-oriented medical record was adopted by many, though often partly.

\section{2 .2 Elstein's diagnosis-directed model}

Whereas Weed's ideas were mainly prescriptive and focused on the documentation process, Elstein developed a descriptive model of medical reasoning, shaped as an iterative, hypothetico-deductive, process similar to the well-known empirical cycle that is used to describe scientific practice. In his model, medical reasoning is an iterative process of cue acquisition, hypothesis generation, cue interpretation, and hypothesis evaluation, directed to a medical diagnosis. In particular, he studied how accurate physicians interpret cues when testing their hypotheses. ${ }^{6}$ In his work, Elstein combined the hypothetico-deductive method of medical practice with the theory of human problem solving from Newell and Simon. ${ }^{4.15}$

What Elstein's and Weed's models have in common, is the idea of medical practice as an iterative process. However, there are also some differences. Weed prescribes how physicians should work, and Elstein describes how they actually work, and both ways are different. Weed emphasises the inductive aspect of problem-driven reasoning (i.e., managing a problem), while Elstein describes medical reasoning as a hypotheticodeductive, diagnosis-directed, activity. Elstein's model of the medical-reasoning 
process is more detailed, as it makes a distinction between hypothesis generation, cues interpretation, and hypothesis evaluation, while Weed's model combines these steps under the single heading assessment. A third difference is the fact that Elstein does not propose the routine generation of a complete initial database before a first assessment should be made. In Elstein's model, the initial database contains sufficient information as soon as an initial hypothesis can be generated.

\section{2 .3 Integration of both models}

It is evident that, in certain aspects, Weed's and Elstein's models are complementary. One of us (PdVR) developed a model of systematic medical practice in which both models are combined. It is presently used to teach systematic medical practice to starting residents. ${ }^{16}$ The model has a problem-driven as well as a diagnosis-directed component. We confine ourselves to a summary description of systematic medical practice according to this integrative model.

The first patient encounter is usually the medical intake, consisting of medical history and physical examination. Medical-history taking is conceived as a very dynamic process of recognising and exploring the PATIENT PROBLEMS. Nondirective phases, in which the patient is uttering his complaints, alternate with directive phases, in which the physician is leading the conversation when testing one or more hypotheses that have emerged. At the end of the intake, the physician reviews the relevant findings (CUES) and makes up a problem list of medical problems. A MEDICAL PROBLEM is defined as the initial constellation (meaningful arrangement) of relevant findings that, according to the physician, is certainly part of one single disease process (i.e., leading to one single DIAGNOSIS).

After completion of this problem list the physician formulates, for each medical problem, one or more HYPOTHESES concerning its cause, its consequences, and possible associations with other MEDICAL PROBLEMS. Each HYPOTHESIS leads to an ACTION PLAN for testing or treatment. These actions result in new CLINICAL DATA that , together with already existing data, are interpreted to confirm or reject the HYPOTHESIS. With this new insight, the physician is able to REFORMULATE the initial medical problem into a new constellation of relevant findings, which may give rise to new HYPOTHESES, which again will be tested, etc., until finally a DIAGNOSIS can be established.

A medical problem should not be confused with a problem presented by the patient, a definition often used in primary care. Instead, it is the re-articulation of the patient's problem into a problem that is manageable for the physician and that serves as a starting point for further medical actions. ${ }^{17}$ The management of medical problems is conceived as a converging process of iteratively reformulating new, more accurate, constellations until finally a diagnosis has been reached. This process of medicalproblem solving is prominently present in the model. An important aspect in this process is the role of relevant findings (CUES): the contribution of clinical findings to the reformulation of medical problems and the role of these reformulations in the generation of further hypotheses or, finally, the establishment of a diagnosis. 


\subsubsection{Rector"s idea of a faithy medical record}

The three above described models reflect the reasoning of one single physician. Since medical practice, including clinical judgement, becomes more and more a collaborative effort, these modcls have becone a too narrow base for describing the entire process of medical practice. In 1991. Rector introduced the concept of a fathful medical record. Prominently present in this concept is the notion of clinical dialogue, which comprises requests, referrals, opinions, and the corresponding responses. In Rector's view, faithfulness to the process of medical practice is the basic requirement of the medical recond: "A faithful medical record should necessarily consist of what clinicians have stated about what they have heard, seen, thought, and done. (...) It should be faithful to the observations, to the decision-making process, and to the clinical dialogue. (...) Information in the medical record is not about what is true of the patient, but what was observed and believed by clinicians." Rector proposed a two-level concept of observations and meta-observations. Observations are persistent, because they keep their value. In Rector's sense, also a diagnosis is treated as an observation. The significance of meta-observations for the medical-care process, on the other hand, is diminishing in time. Decisions and dialogues are part of this level. Problems and plans are examples of decisions. Orders and reports, but also referrals and expert opinions, are examples of clinical dialogues.

We have adopted Rector's idea of faithfulness. In particular, we wellcome the notion of the clinical dialogue as a logical extension to the model of individual medical practice. In the next paragraph, we describe a model of collaborative medical practice in which the clinical dialogue is tightly interwoven with the other elements of systematic medical practice.

\subsection{A conversation-based model of medical practice}

In their conversations-for-action concept, Winograd and Flores maintained that information is the result of a conversation between two human beings. ${ }^{18}$ In agreement with this concept, all medical data can be considered the result of a conversation or, to speak in Rector's terms, a clinical dialogue. This understanding of conversations as the exclusive source of (original) information forms the basis of our new model of collaborative medical practice. Implicit to this understanding, we replace Rector's distinction between persistent observations and transient meta-observations (decisions and dialogue) by a distinction between essential actions (observations and decisions) and informative actions (dialogue). Essential actions change the "object world" (read: the patient's health); informative actions communicate these changes. Any information about observations and decisions is generated as part of a conversation or clinical dialogue. With other words: the medical record merely consists of clinical dialogue. In the next sections of this paragraph, we further explain this new model of collaborative medical practice and its consequences for medical-record keeping in particular. 


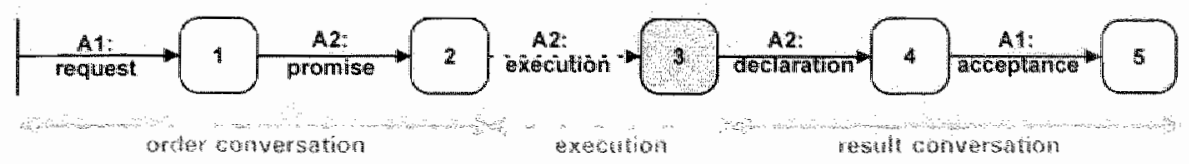

Figure 5-1:

The transaction concept Rounded boxes are transaction states, arrows denote transitions between these states. Only the successful path of the transaction is shown.

\subsubsection{The transaction concept}

Winograd and Flores described the generation of information from a languagephilosophical point of view, as a series of negotiations called conversations for action. Based on this approach of information processing, one of us (JD) has developed an experimental method and associated terminology for the description of business processes and the development of information systems, called Dynamic Essential Modelling of Organisations (DEMO). ${ }^{19}$ In the DEMO terminology we can represent the process of medical practice in a model that includes observations, decisions, and dialogues.

A fundamental principle of DEMO is the transaction concept, which describes the elementary business process as an essential action encapsulated by two conversations between the actors $A 1$ and $A 2$ (Figure 5-1). A transaction starts with a request from A1 to A2 to perform an essential action, e.g., to establish a diagnosis. This order conversation is completed after A2 promises A1 to perform this action. After having finished this action, $\mathrm{A} 2$ reports its result back to $\mathrm{A} 1$ in a result conversation, which is completed after A1 accepts the result. In DEMO, an actor is not considered a concrete person but an abstract role. An actor role can be personified by one or more persons, and a single person can play one or more actor roles. This notion of actors is important

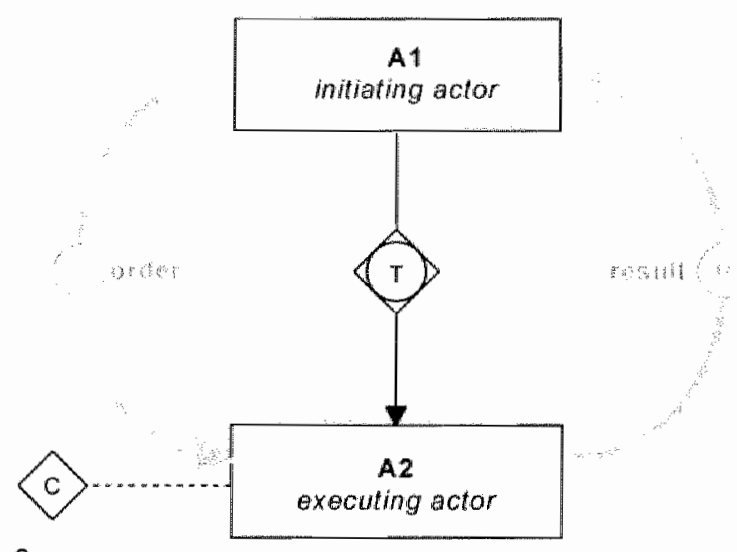

Figure 5-2:

The general pattern of a transaction, described as an interaction between an initiating and executing actor. The flow of conversations is in gray.

$A=$ actor; $T=$ transaction; $O=\operatorname{order}_{;} R=$ result; $C=$ cues. 


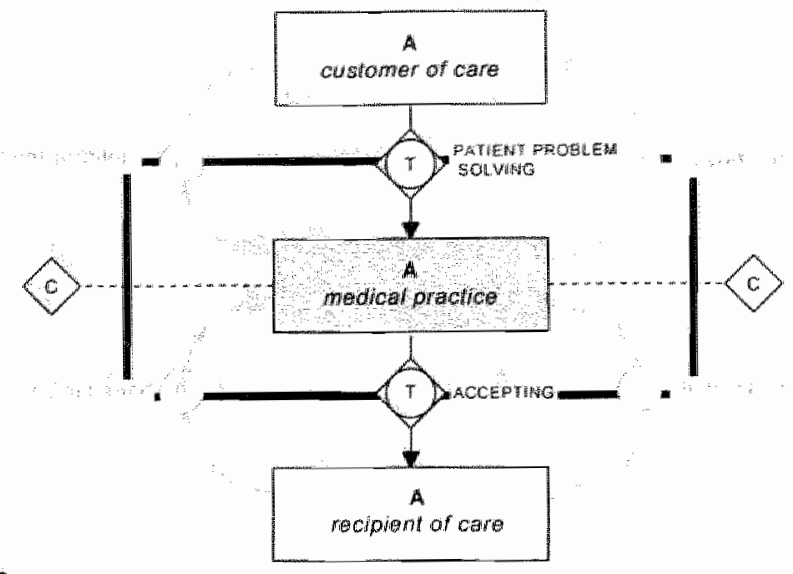

Figure 5-3:

Globall conversation model of medical practice. The fat black rectangle denotes the system's border, a thin box represents an actor. Elementary actors are white, global actors are gray. The flow of conversations is presented as thick gray arrows.

$A=$ actor; $T=$ transaction; $O=$ order; $R=$ result; $C=$ cues.

for the use of DEMO, because it implies that DEMO does not make a difference between inter-individual and intra-individual conversations. Figure 5-2 presents the same transaction in another graphical format. It demonstrates that $\mathrm{A} 1$ plays the role of initiating actor and A2 the role of executing actor. Apart from the order from A1, actor A2 will probably need more information to perform his task. If it is A2's task to establish a diagnosis, for example, he will need CUES, e.g., some laboratory results and a pathology report.

The transaction concept can be used to describe any elementary business process. If we consider medical practice as a business process - actually, it is the primary (business) process of health care - any essential medical action (observations as well as decisions) can be described as the execation phase of a transaction, and hence is encapsulated by two conversations (clinical dialogues).

\subsubsection{A conversation model of medical practice}

DEMO makes use of an interaction model to show which transaction types exist in the business system under concern, and to determine the initiating and executing actors of each transaction type. In our case, the system under concern is medical practice. To better illustrate our purpose, we prefer the term conversation model to the term interaction model.

Global conversation model (Figure 5-3) - The global model shows the conversations (interactions) of the system (i.e., medical practice) with its environment while its inner structure remains hidden. The global actor MEDICAL PRACTICE maintains a conversation with two external actors: CUSTOMER OF CARE and RECIPIENT OF CARE. Both these external actor roles are usually played by the patient. The model can be explained as follows. A patient, in the role of CUSTOMER OF CARE, starts an order conversation by expressing a reason for consultation. The usual reason is a health complaint. The actor MEDICAL PRACTICE, played by a physician or a health-care team, promises to solve the 


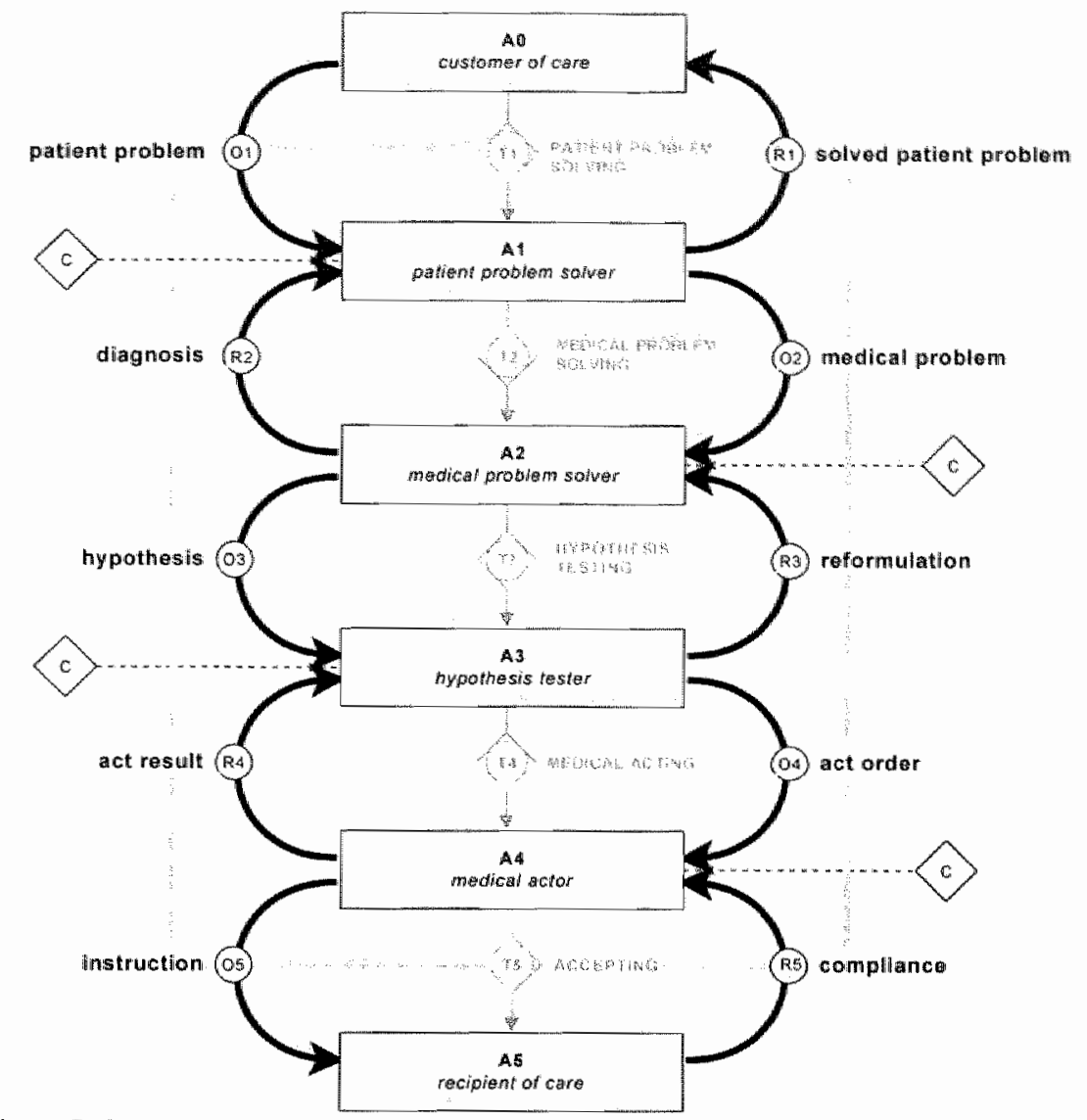

Figure 5-4:

Detailed conversation model of medical practice. The flow of obey conversations is presented as thick black arrows. Cues may come either from inside or from outside the system. To reduce the model's complexity, only external cues are presented.

$A=$ actor; $T=$ transaction; $O=$ order; $R=$ result; $C=$ cues.

problem. To achieve this, MEDICAL PRACTICE must carry out observations, procedures, or treatments that need compliance from the side of the patient in the role RECIPIENT OF CARE. When MEDICAL PRACTICE has obtained solution for the CUSTOMER-OF-CARE'S problem, the result conversation is started in which the CUSTOMER OF CARE is asked to "accept" this solution, e.g., the patient is asked whether he has been treated satisfactory.

Detailed conversation model (Figure 5-4) - In the detailed model, also the inner structure of medical practice is specified: the elementary actors and their interactions (conversations). The inner structure should represent the construction (working mechanism) of medical practice ${ }^{20}$ Thus, we have designed a detailed conversation model consistent with the description of medical practice we gave in Paragraph 5.2.3. Medical practice is represented as a composition of four elementary actors. Each actor directs an actor lower in the model and uses the results provided by this lower actor. CUES may be needed by any actor and originate either inside or outside medical 
practice. To reduce the complexity of picture, only extemal cues are presented. Figure 5-4 can be explaned as follows:

71. Actor A1 serves as the "face" of medical practice, namely as the patient's hostess or advocate. His task is to solve the PATIENT PROBLEM. To reach a SOLUTION for the PATIENT'S PROBLEM, A1 must know which DIAGNOSES can be determined.

12. Hence, he formulates one or more MIEDICAL PROBLEMS and charges A2 to establish a DIAGNOSIS for each MEDICAL PROBLEM.

Tr. To reach a DIAGNOSIS, A2 formulates one or more HYPOTHESES about the cause and consequences of each MEDICAL PROBLEM, and about possible associations with other MEDICAL PROBLEMS. These HYPOTHESES are then tested by A3, and their (dis)iconformation is expressed in a REFORMULATION of the MEDICAL PROBLEM, as the first converging stage towards a DIAGNOSIS. Further REFORMULATIONS will follow as A2 repeatedly formulates new HYPOTHESES, unitil A2 has enough information to establish a DIAGNOSIS

14 To test a HYPOTHESIS, A3 generates an action plan, consisting of one or more ACT ORDERS for observations, interventions, and other medical actions. These actions are performed by A4 and their RESULTS are reported back to A3.

is. To complete a medical act, A4 gives the patient - in the role of recipient of care INSTRUCTIONS to obtain COMPLIANCE to achieve a proper result.

In the description of systematic medical practice given in Paragraph 5.2.3, a MEDICAL PROBLEM was defined as the first of a series of constellations. In Figure 5-4, however, MEDICAL PROBLEMS and their REFORMULATIONiS are positioned at different levels of the model. This distinction needs some explanation. In DEMO, an iterative process is represented as a nested pair of transaction types: a main transaction that delimits the start and completion of the entire process, and a sub-transaction for the iterations. Each result from a sub-transaction is then used as a cuE to assess the progress of the main transaction. From the perspective of actor A2 in Figure 5-4, a MEDICAL PROBLEM is the start of a process that must finally result in a DIAGNOSIS. REFORMULATED constellations, received iteratively from $A 3$, are used as new CUES to assess whether a DIAGNOSIS can be obtained. This does not imply, however, that MEDICAL PROBLEMS and their REFORMULATIONS are completely different things. They only play different roles in the process of medical practice.

\subsubsection{Interpretation of the conversation model}

The detailed conversation model of medical practice presented in Figure $5-4$ is of a high level of abstraction. This abstraction makes it universally applicable, but perhaps difficult to project to concrete situations. For a proper interpretation of the conversation model, one should take notice of the following abstractions, which we have illustrated with some examples:

Abstraction 1: Persons and professions - The elementary actor roles within medical practice (A1.A4) can be played by different persons from different disciplines and professions. For example, if an internist is confronted with a patient with shortness of breath, he may call in a cardiologist to exclude any pathology of the heart. This cardiologist, in turn, may order the clinical laboratory to perform some tests. In other situations, for example in general practice, one and the same person may perform all 
actor roles. To avoid premature personification of actor roles, it is better to think of medical practice as an organisation and of actors as decision levets in this organisation. Also the two external actor roles, usually played by the patient, are abstractions. In some situations, like medical-history taking from an infant, the patient may be represented by one of his parents. Apart from that, a distinction must be made between the patient as subject - in either of the extemal actor roles - and the patient as object. All elementary actors notice the patient as object, while only A1 and A4 deal with the patient as subject.

Abstraction 2: Phase of medical practice - The conversation model does not distinguish between intake, diagnostic phase, themapeutic phase, and discharge. Instead, it is applicable to all phases of care. During intake, in particular medical-history taking, the physician constantly tries to re-articulate the PATIENT'S PROBLEM into a MEOICAL PROBLEM that is manageable for him. Each time a HYPOTHESIS about possible causes, consequences, or relations with other MEDICAL PROBLEMS comes to mind, he formulates some additional questions and asks the patient to answer them. These answers contribute to the acceptance or rejection of that HYPOTHESIS. These activities usually do not take more than a few seconds and are repeated several times during one single session of medical-history taking.

The only difference between diagnostic and therapeutic phase lays in the character of the information that is communicated between the elementary actors. ACT ORDERS and RESULTS may refer to diagnostic procedures but also to therapeutic interventions. $A$ HYPOTHESAS may have the character of a differential diagnosis, but can also imply a prognosis about the effect of a possible therapy. In this context, even the term DIAGNOSIS should be interpreted broader than its usual definition: determination of the nature of a disease. ${ }^{21}$ When used in the therapeutic phase, the term DIAGNOSIS will denote something like: determination of the outcome of a treatment. For example, after having completed the DIAGNOSIS "acute gastro-enteritis caused by campylobacter", the next DIAGNOSIS to pursue is "physical state after gastro-enteritis, successfully treated with tetracycline". Likewise, MEDICAL PROBLEMS may refer to diagnostic as welli as therapeutic management problems. In this sense, "MOPP-treatment for Hodgkin's disease, stage $I \mathbb{1}-\mathrm{A}$ " is a MEDICAL PROBLEM, which may (hopefully) result in the DIAGNOSIS "Hodgkin's disease, stage 11 -A, complete remission after MOPP-treatment". When different MEDICAL PROBLEMS co-occur, it is possible that some are diagnostic and other therapeutic.

Abstraction 3: Medical documentation - In DEMO, conversations (dialogues) represent the content, not the format, of information. ${ }^{19}$ The conversation model does not specify how the information has to be documented. Information can be stored in any fashion: on paper or electronically, as free text or in coded format. Information may not even be recorded at all, which is the case for most HYPOTHESES generated during history taking and also for the many times COMPLIANCE is asked from the actor recipient of care (the fact that the patient gave blood for testing is documented, the fact that he raised his right arm for examination is not). 


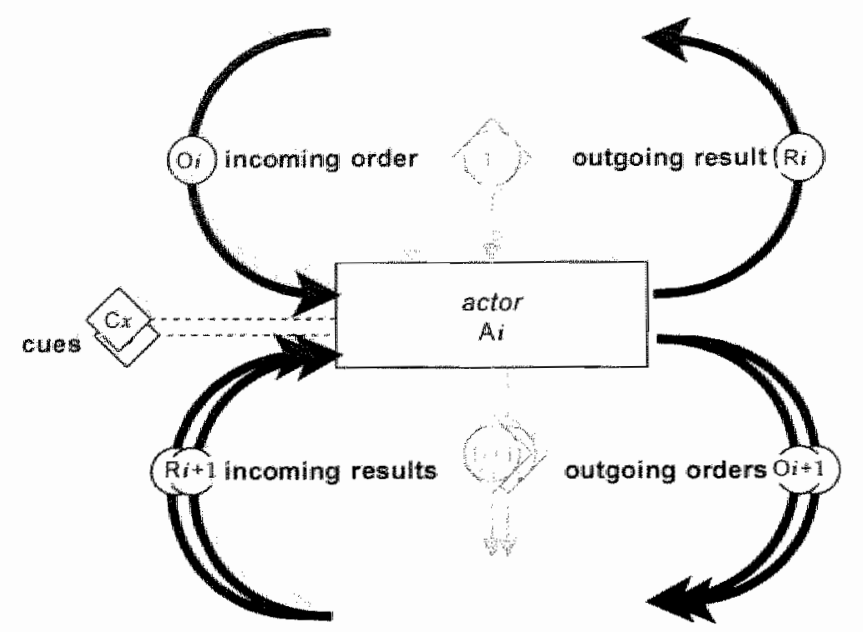

Figure 5-5:

Information processing of a general actor. The flow of conversations is presented as thick grey arrows

$A=$ actor; $T=$ transaction; $O=$ order $_{;} R=$ result; $C=$ cues.

\subsection{Implications for the structure of the medical record}

In Paragraph 5.3 we have presented a model of the business process of medical practice with transaction as its elementary concept. A transaction generates information about the object world, namely the patient's health and treatment. In this paragraph we describe a data model for storing information about the transaction process itself, namely the process of medical practice, which concerns the subject world. In Chapter 4, we introduced the process view as the reflection of this process in medical record.

Let is first reconsider a single actor as an information processor. Figure 5-5 shows that actor $A 1$ is directed by an INCOMING ORDER $\left(O_{1}\right)$ and that its task is to achieve an OUTGOING RESULT $\left(\mathbb{R}_{i}\right)$. When performing its task, A1 needs existing clinical data as CUES $\left(\mathrm{C}_{x}\right)$ about the patient, his disease, and the treatment. If existing lindings are not sufficient, A1 will generate OUTGOING ORDERS $\left(\mathrm{O}_{i+1}\right)$ to other actors, in order to obtain new CUES in the form of INCOMING RESULTS $\left(R_{i+1}\right)$.

The pattern of information processing demonstrated in Figure 5-5 is general for each elementary actor and is nothing more or less than the creation of links (relations) between medical conversations. Four types of process links can be distinguished (Table 5-1).

- Completion link - This link sets the relation $\left\langle\mathrm{O}_{i}\right\rangle$ asks for $\left\langle\mathrm{R}_{i}\right\rangle$, between an INCOMING ORDER and the OUTGOING RESULT it brings about. It approves the completion of a transaction. 
Table 5-1:

Relations representing four types of process links.

\begin{tabular}{|c|c|}
\hline \multicolumn{2}{|c|}{$\angle \mathrm{O}_{\mathrm{p}}>$ asks for $\angle \mathrm{R}>$} \\
\hline $\begin{array}{l}\text { patient problem } \\
\text { medical problem } \\
\text { hypothesis } \\
\text { act order } \\
\text { instruction }\end{array}$ & $\begin{array}{l}\text { solved patient problem } \\
\text { diagnosis } \\
\text { reformulation } \\
\text { act result } \\
\text { compliance }\end{array}$ \\
\hline \multicolumn{2}{|c|}{$\left\langle\mathrm{O}_{i}\right\rangle$ is the indication for generating $\left.<\mathrm{O}_{i+1}\right\rangle$} \\
\hline $\begin{array}{l}\text { patient problem } \\
\text { medical problem } \\
\text { hypothesis } \\
\text { act order }\end{array}$ & $\begin{array}{l}\text { medical prablem } \\
\text { hypothesis } \\
\text { act order } \\
\text { instruction }\end{array}$ \\
\hline \multicolumn{2}{|c|}{$<\mathrm{R}_{i+1}>$ contributes to the assessment of $<\mathrm{R}_{p}>$} \\
\hline $\begin{array}{l}\text { compliance } \\
\text { act result } \\
\text { reformulation } \\
\text { diagnosis }\end{array}$ & $\begin{array}{l}\text { act result } \\
\text { reformulation } \\
\text { diagnosis } \\
\text { solved patient problem }\end{array}$ \\
\hline \multicolumn{2}{|c|}{$\left\langle\mathrm{C}_{x}>\right.$ is used as motivation for $\left\langle\mathrm{C}_{y}\right\rangle$} \\
\hline $\begin{array}{l}\text { existing cues } \\
\text { existing cues } \\
\text { existing cues } \\
\ldots\end{array}$ & $\begin{array}{l}\text { new order or result } \\
\text { new order or result } \\
\text { new order or result } \\
\ldots\end{array}$ \\
\hline
\end{tabular}

- Indication link - This links sets the relation $\left\langle\mathrm{O}_{i}\right\rangle$ is the indication for generating $\left\langle\mathrm{O}_{i+1}>\right.$, between the INCOMING ORDER and an OUTGOING ORDER to obtain new CUES. It indicates the reason why the OUTGOING ORDER is generated, namely to support the transaction started with $\mathrm{O}_{i}$.

- Assessment link - This link sets the relation $\left\langle\mathrm{R}_{i+1}>\right.$ contributes to the assessment of $\left\langle\mathrm{R}_{i}\right\rangle$, between an INCOMING RESULT and the OUTGOING RESULT. The INCOMING RESULT is used as a new cue to assess whether the actor has completed its task and can generate an OUTGOING RESULT.

- Motivation link - This link sets the rellation $\left\langle\mathrm{C}_{x}\right\rangle$ is used as a motivation for $\left\langle\mathrm{C}_{y}\right\rangle$, between an ORDER or RESULT and the cues that are consulted. Unlike an assessment link, a motivation link does not necessarily involve a cue specifically generated for this purpose. Also already existing data can serve as cues for motivating an order or result. ${ }^{\text {a }}$

a DEMO does not distinguish between already existing cues and new cues. Yet, we will make this difference in the process view. We feel that - at least for educational purposes - it is important to explicitly show the effect of a new, specifically generated, cue in the assessment of a current task. This effect can be recorded as an assessment link, which is - in terms of DEMO - a special instance of a morivarion link. 


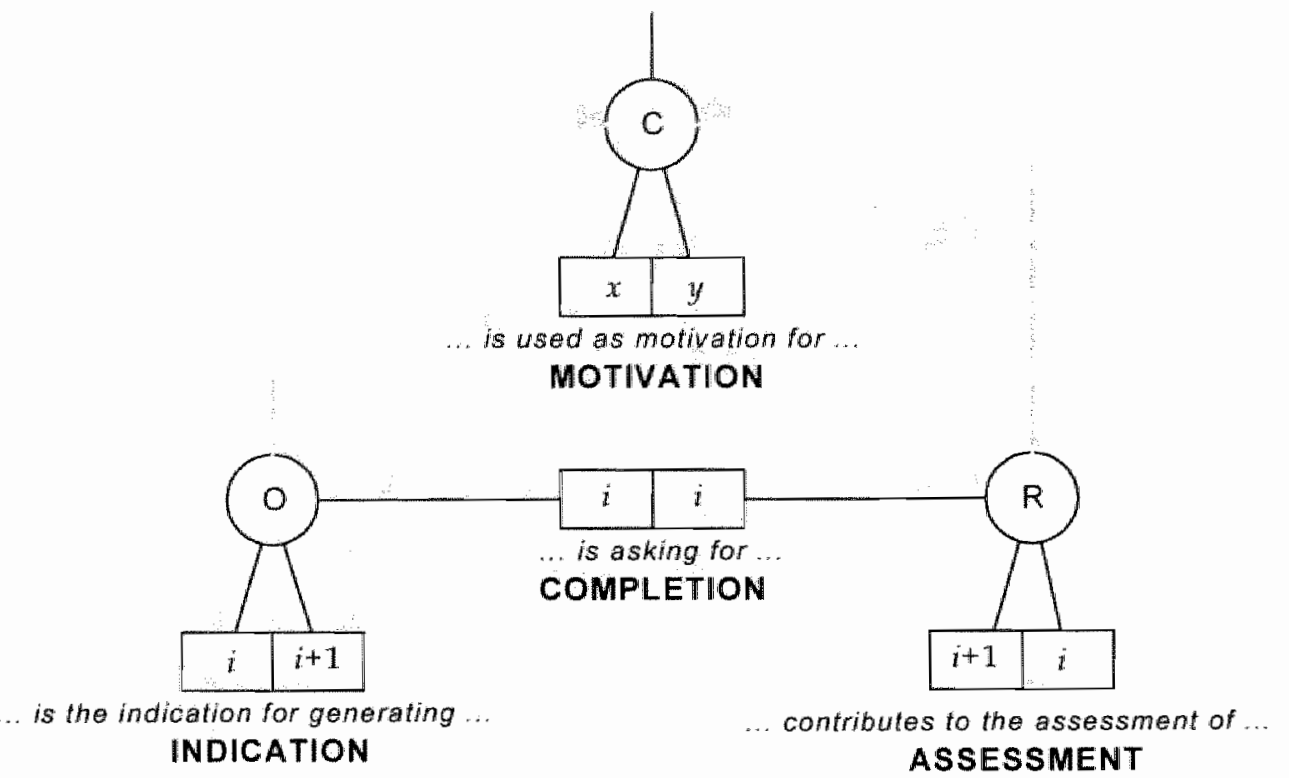

Figure 5-6

Data model of the process, view. $O=$ arder; $R=$ result; $C=$ cues.

The numbering of elements is conforming Figure 5-5. Graphical conventions are according to the N:AM-method of information analysis.

In Figure 5-6, these process links are presented in a conceptual data model of the process view. In this model, each bi-compartmental box represents a (binary) process link between two medical data. Each compartment of a box is connected to its domain, represented by a circle. The grey arrows denote that both orders and results are subdomains of the domain of CUES. Via the domain of cues, the process view is connected with the rest of the data model of the medical record.

Together, these process links contain information about how the physician has used the available medical data during the delivery of care. Completion links mark the progress of medical practice, while indication links, assessment links, and motivation links express the ratio behind medical practice. The process view as a whole provides a reconstruction of the process of medical practice.

\subsection{Discussion}

Weed's SOAP-structure was the first attempt to improve this aspect of medical documentation. To a certain extent, SOAP is comparable with the process view as described above: the relation between observations ( $\mathrm{SO}$ ) and subsequent assessment $(\mathrm{A})$ is an assessment link, the relation between assessment (A) and subsequent plan (P) is an indication link, and the relation between plan (P) and following observations ( $\mathrm{SO}$ ) is a completion link. However, SOAP has some limitations. First, the actors A2 and A3 are compressed to one actor - which we can call CLINICAL JUDGEMENT - hence grouping HYPOTHESES and REFORMULATIONS to one single category ASSESSMENT (A). Second, 
observations ( $\mathrm{SO}$ ) and plans (P) concern only ORDERS and RESULTS Of MEDICAL ACTING, while process links can be generated at any level of the conversation model. Third, SOAP does not represent motivation links between existing data and assessment.

The use of SOAP has never become popular. For a long time, Weed's own PROMIS was the only electronic medical-record system in which SOAP was implemented. However, the need for insight in the process of medical practice is growing. ${ }^{3}$ Only recently, some researchers ventured upon the adoption of a process view as part of the electronic medical record. ${ }^{22.26}$ Compared with these initiatives, our approach is unique in the fact that its explicit purpose is to also support shared medical care.

\subsubsection{Added value for the design of electronic medical-record systems}

An adequate view on the process of medical practice, which makes the delivery of care more transparent, may facilitate the co-ordination of care activities, case-based medical education, medical audit, utility management, and can also serve legal purposes. In his idea of a faithful medical record, Rector identified the need to include not only medical observations but also decisions and clinical dialogues in the medical record. ${ }^{7}$

In present health care, however, many medical actions and decisions are not accomplished by individuals any more, but are generated as a collective responsibility. Hence, the need to consider medical actions and decisions, somehow, in relation to conversations is evident. This need was recognised by Frisse et al., who advocated the use of conversations as the central metaphor for handling patient records, because conversations would reflect best the work flow in a clinical setting: "It allows one to clearly identify who talked to whom and when, and it retains a record of tasks in a way that is useful for retrospective analysis from both a utilization review and a legal perspective". ${ }^{1}$ The conversation model is a systematic description of shared medical practice based on the above mentioned metaphor. The data model of the process view as described can provide an extension to the medical record's database that makes such retrospective analyses possible.

Completion links between orders from physicians and results from auxiliary services can provide valuable information for hospital logistics and utilisation review. Hence, such links are usually recorded in the order-communication module of existing hospital-information systems. The orders in our conversation model, however, have not always logistic consequences, since they may be executed by the same person as by whom they were initiated. Usually, these transactions do not have logistic consequences, hence nobody has ever felt the need, so far, to record their progress. Yet, we have reasons to believe that clinicians themselves would benefit from such information. A survey by one of us (HT) among 85 physicians in an academic hospital showed, that a majority of them considered their paper-based, traditionally structured, medical records complete enough with respect to the registration of diagnostic procedures and therapeutic interventions, but incomplete with respect to the registration of decisions, orders, and plans. According to most of them, these elements should also be included in the medical record (Chapter 2). Apparently, physicians have insufficient insight in the operation of their orders. The provision of completion links would satisfy this need. 
Physicians seem to feel also the need for information about the rationale behind medical decisions of others. Indication links, assessment links, and motrvation links contain valuable information about this rationale. These links are the testimony of what Wyatt expressed as follows: "The significance of clinical data can be measurad along its relevance to medical decisions". 27

The idea of extending the medical record with a meta-level of links between medical data that represents the clinical process was also described by others. ${ }^{22.28 .29}$ We found no evidence, however, of the implementation of this idea in any current electronic medical-recond system. Problems with the logistics of the user-interface are probably one of the causes. 22 The design of an appropriate user-interface for the creation and presentation of process views is a challenge that needs further study. For the time being, the registration of free-text narrative stories, implicitly embedding medical data into their clinical context, seems the only possible rough sketch of both whe "patient as text" (clinical data) and the "clinician as author" (the meta-level). ${ }^{30}$

\subsubsection{Robustress of the conversation model}

There is a trend of substituting health-care activities from clinical care to ambulatory care, from clinical care to primary care, from specialty care to sub-specialty care. Such a shift of responsibilities will have major consequences for the design of future information systems in health care. Lamberts foresees a future in which primary care will be the setting where all information comes together ${ }^{31}$. Whether this will come true or not, the abstract character of the elementary actors makes our conversation model of medical practice robust for any substitution of responsibilities between persons, disciplines, or professions. What will only change are the players of actor roles, not the actor roles themselves. As long as the function of medical practice does not change and why should it - the conversation model will remain a valid description of the way this practice is accomplished.

The function of medical practice would change, however, in a future as recently sketched by Kassirer, in which the use of information technology, together with the emancipation of the patient, would eventually lead a decreasing role of the physician in medical practice. The well-informed patient of the future would seek advice first from the computer; since computers might surpass humans in making decisions, leaving the delivery of care to humans. ${ }^{32}$ Our conversation model does not permit that computers substitute human beings in making decisions. The transaction concept described in Paragraph 5.3.1 implies, that information is always the product of a dialogue between humon beings. They are responsible and accountable for the tasks they have accepted, not the machines that may support them. For example, a clinical chemist can never deny responsibility for an incorrect test result by referring to a failing blood analyser. Why else does he have to authorise, also, computer-generated test results?

In this sense, our actor concept should not be mixed-up with Rector's agent concept which he used in association the faithful medical record. According to Rector, each observation has to be attributed to an agent, which is defined as the person or device that can originate observations. ${ }^{7} \mathrm{~A}$ human agent can be replaced by a computer, a human actor can only be replaced by another human, but never by a computer. If in 
the future, the patient would first turn to the computer to seek help, it is not the computer that plays for the doctor, but the patient himself.

\subsubsection{Other possible uses of the conversation model}

The immediate reason for us to develop a conversation model of systematic medical practice was to obtain a framework for the development of the process view in an electronic medical record. We believe, however, that our model can also be used for other purposes, hence it would be worthwhile to explore its the added value for these purposes.

It may be used to measure which information physicians (or students) use when taking decisions, or to trace back a series of medical decisions to determine the underlying rationale of a treatment strategy, as part of medical audit. Also in the domain of medical informatics, the conversation model of medical practice may have an added value more than only for building medical record systems. For example, the conversation model may serve as a useful reference for information planning, and as such it would be a welcome extension to existing information-reference models as they have been developed and applied in The Netherlands. ${ }^{33.34}$ In these informationreference models, medical practice is still a white spot in an otherwise structured model of the health-care organisation.

As already noticed, misunderstandings about the possible use of decision-support systems in medical practice are widespread. ${ }^{32}$ With this conversation model as a reference framework, it may be possible to classify decision-support systems in terms of the elementary actors that they support. In this sense, a distinction can be made between decision-support systems for patient-problem solving, medical-problem solving, hypothesis testing, and medical acting. Whether this distinction is useful in practice, needs further investigation.

Another interesting, but speculative, possibility is to use the conversation model in projects concerning substitution of care and other forms of business-process redesign, namely for redistributing responsibilities among professionals with overlapping competence. The actor concept, being an abstraction from persons and professions, may facilitate a rationalistic distribution of tasks in concrete settings, independent of existing traditions. In the same sense, the model might be used as a reference to support future projects concerning the substitution of health care activities. The DEMO-method has already demonstrated its use for similar projects in non-medical organisations, in the context of business-process redesign. ${ }^{35}$ inally, the converstion model might also serve as an interesting perspective for medico-ethical and medicolegal studies. At least, in the ambivalent role of the patient in our model - as both customer and recipient of care - we recognise some of the uneasiness in the patientdoctor rellation.

\subsection{Conclusion}

An electronic medical record that includes information about the delivery of medical care has to be based on a sound model of the process of medical practice. We have 
demonstrated, that the existing models of Weed, Elstein, and Rector are complementary to each other. We have presented a model of systematic medical practice in which these models are combined. Essential characteristics of this conversation model are (1) the use of conversations as the exclusive medium for expressing the results of observations and decisions, and (2) the representation of humans as actors that are abstracted from concrete persons, disciplines, and professions. We used the conversation model for preparing the specifications of the process view for the database of an electronic medical-record system, in the notion that a process view retains promises for medical education and medical audit. We maintain, that the conversation model is robust against organisational changes like substitution of care.

\subsection{References}

1. Frise ME, Schnase JL, Metcalfe ES. Models for patient records. Acad Med 1994; 69:546-50.

2. American College of Physicians. The role of the future general internist defined. Ann Intern Med $1994: 121: 616-622$.

3. Dick RS, Stein EB. The computer-based patient record - an essential technology for health care. Washington DC: National Acadeny Press, 1991.

4. Van Londen I. Dutch health care - a study in purple. Lancet 1996;347:1229-1231.

5. Weed LL. Medical records that guide and teach. N Engl J Med 1968; 278:593-600,652-657.

6. Elstein AS, Shulman LS, Sprafka SA. Medical problem solving: an analysis of clinical reasoning. Cambridge, MA: Harvard University Press, 1978.

7. Rector AL, Nowlan WA, Kay S. Foundations for an electronic medical record. Methods Inf Med $1991 ; 30: 179-86$

8. Weed LL. Medical records, medical education, and patient care. Chicago: Year Book Medical Publishers, Inc., 1969.

9. Weed LL. Quality control and the medical record. Arch Intern Med 1971; 127:101-105.

10. Goldfinger SE. The problem-oriented record: a critique from a believer. N Engl J Med 1973; 288:606-8.

11. Feinstein AR. The problems of the "Problem-Oriented Medical Record". Ann Intern Med 1973; $78: 751-762$.

12. Donnelly $W d$, Hines $\mathbb{E}_{,}$Braumer DJ. Why SOAP is bad for the medical record. Arch Intern Med $1992 ; 152: 481-84$.

13. Feinstein AR. Quality of data in the medical record. Comput Biomed Res 1970; 3:426-35.

14. Newell A, Simon HA. Human problem solving. Prentice-Hall. Englewood Cliffs, NJ: PrenticeHall, 1972 .

15. Berg M. Rationalizing medical work - Decision support techniques and medical practices. Manstricht, NL: Maastricht University, 1995.

16. de Vries Robbe PF. Stuyt. PMJ, van der Meer JWM. [Onderwijs in methodisch denken in de praktische geneeskunde] (in Dutch). In: Metz JCM, Scherpbier AJJA, van der Vleuten CPM, eds. Medisch onderwijs in de praktijk. Assen, NL: Van Gorkum, 1995:58 68 .

17. Berg M. The construction ol medical disposals - Medical sociology and medical problem solwing in clinical practice. Sociol Health \& IIness 1992; 13:151-180.

18. Winograd T, Flores FM. Understanding computers and cognition. Norwood, NJ: Ablex Publishing Corporation. 1986.

19. Dietz JLG. The essential system model. Lecture Notes in Computer Sciences. Vol. 498: Springer Verlag, 1991. 
20. Dietz JLG. [Wat doen computers als ze iets zeggen?] (inaugural college, in Dutchy, Department of Information Systems, Faculty of Technical Mathematics and Informatios. Delft, NL: University of Delf, 1996.

21. Dorland's illustrated medical dictionary. Philadelphia: WB Saunders Company, 1981.

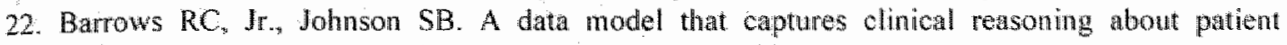
problems. Proc Annu Symp Comput Appl Med Care 1995:402-5.

23. Poon $A D$, Fagan $L M$, Shortiffe $E H$. The PEN-Ivory project: Exploring user-interface design for the selection of items from large controlled vocabularies in medicine. J Am Med Inform Assoc $1996 ; 3: 168-183$.

24. van Ginneken $\mathrm{AM}$, Stam $\mathrm{H}$. Can one patient record accommodate the diversity of specialized care? Proc Annu Symp Comput Appl Med Care 1995:406- 10.

25. Assimacopoulos $A_{n}$ Borgazzi $A$. An electronic patient record combining free text and ooded nomenclature: application to nursing process, Proc Medinfo92, 1992. North-Holland.

26. Trace D, Naeymi Rad F, Haines $D$, et al. Intelligent Medical Record--entry (IMR-E). J Med Syst $1993 ; 17: 139=51$.

27. Wyatt JC. Clinical data systems, Pant I: Data and medical records. Lancet 1994; 344: : $543-7$.

28. van Ginneken AM, Stam H, Moorman PW. A multi-strategy approach for medical records of specialists, Proc Medinfo' 95,1995 . North-Holland.

29. Kluge EHW. The medical record: narration and story as a path through patient data [editoriall. Methods Inf Med 1996; 35:88-92.

30. Kay $\mathrm{S}$, Purves IN. Medical records and other stories: a narratological framework. Methods Inf Med 1996;35:72-87.

31. Lamberts $H$, Hofmans-Okkes $\mathbb{1}$. The generic patient record: an alliance between patient documentation and medical informatics. Methods Inf Med 1996; 35:5-7.

32. Kassirer JP. The next transformation in the delivery of health care. N Engl J Med 1995; 332:5254.

33. Geurts-de Haas GE, van Oorschot HPF, van Vondel H. [Het ziekenhuisinformatiemodel] (in Dutch). Utrecht, NL: NZI, 1984.

34. ten Hoopen AJ, Nutma $J$, de Vries Robbé PF. Information modelling in the Dutch health care. Medical Informatics Europe 1991?:694-700.

35. Dietz JLG. Modelling business processes for the purpose of redesign. In: Glasson BC, et al., eds. Business process re-engineering: information systems opportunities and challenges: Elsevier Sciences, 1994. 


\title{
6. An experimental electronic medical- record system with multiple views on medical narratives
}

\author{
H.J. Tange ${ }^{a}$, V.A.B. Dreessen ${ }^{a}$, A. Hasman ${ }^{a}$, H.H.L.M. Donkers ${ }^{b}$ \\ " Department of Medical Informatics, Maastricht University, \\ ${ }^{b}$ Department of Computer Sciences, Maastricht University.
}

In this chapter we describe a prototype electronic medical-record system that is developed specifically to facilitate searching through medical narratives, as part of a preparatory study to the development of a medical workstation. This system contains a searching structure in which source view, problem view, time view, and process view are combined. We describe the structure of the database and the user-interface. A second design goal was to prepare the system for conducting research with respect to the use of the medical record. We address the kind of test designs the system can support and describe the research module that was implemented. We also describe a study we conducted with the system and report part of its results. We discuss the significance of this medical-record system compared to systems in the literature and address some technical and medical limitations we experienced and some future plans with the system.

Submitted for publication 
Chapter 6 


\subsection{Introduction}

The Department of Medical Informatics of Maastricht University plans to design a medical workstation that supports the hospital physician in all his activities. At the moment, preparatory studies are conducted concerning the use of voice input (pathology), electronic consultation of practical guidelines (surgery), and the facilitation of consulting literature databases (several medical domains). ${ }^{1-3}$ Here, we report a fourth preparatory study, concerning the requirements of the medical record as a retrieval tool for direct medical practice.

The process of information retrieval from the medical record can be divided into two steps. First, the user has to search through the medical record - guided by its internal structure - to select the paragraph that might contain relevant information. Second. he has to read the content of this paragraph to retrieve the information needed. We decided to pay further attention to the organisation of the medical record, in order to obtain the optimal searching structure for physician-gathered medical narratives, i.e., medical history, physical examination, and progress notes. Our first goal was to develop a prototype electronic medical-record system that can accommodate medical narratives with different structures. Since much research is still necessary to determine the optimal characteristics of electronic medical-record systems, we also decided, as a second goal, to extend this prototype system with a research module. In particular, this research module was used to study the effect of using medical narratives with different granularities on the speed, completeness, and appreciation of information retrieval. The prototype system had to be adapted for this purpose.

In Paragraph 6.2 we explain our approach of the structure of medical narratives and also address some other design considerations. In Paragraph 6.3 we present the most characteristic features of the prototype system. In Paragraph 0 we describe how we compared the ease of information retrieval from medical narratives with three different granularities and present some results of this evaluation. A more comprehensive description of the organisation of medical narratives was given in Chapter $4_{\text {, and }}$ the comparison between different organisations of the electronic medical record will be more extensively described in Chapter 7 .

\subsection{Design considerations}

From descriptions of existing EMR-systens, we deduced three characteristics that are meant to facilitate the retrieval of information from the medical record in general, and from the narrative parts in particular: (1) Stability of the human-computer interface by providing visual cues on fixed positions on the screen, thus enabling the user to follow a reading strategy of pattern recognition when consulting the medical record. ${ }^{4.5}$ (2) Facilitation of searching, by the use of flowsheets and other organising principles of medical data. ${ }^{6.7}$ (3) Facilitation of reading, by presenting medical narratives as natural prose, even if it has to be generated from coded data. ${ }^{58412}$ In the design of our EMRsystem, we took all these facilitating aspects into account. The screen design had to be 
as simple and stable as possible and it was decided to present medical narratives as free text. But above all, the system design focused on the searching structure of medical natratives.

\section{2 .1 Facilitation of searching the presentation structure of medical narratives}

The medical record is a repository of patient information. It can be regarded as a compilation of indexable items and paragraphs that contain clinical information of a certain type, recorded at a certain time. Paragraphs can be ordered in time and also organised by lype, namely according to their source and according to the problems they are relevant for. Hence, each paragraph can be approached from a sourceoriented, problem-oriented, and time-oriented view (see Chapter 4). Furthermore, paragraphs can be linked to one another according to the role they play in the medical care process concerning a specific patient. Four kinds of process links can be distinguished: completion links to connect results with their initiating orders, indication links to give the reason of orders, assessment links to give the reason of results, and motivation links to illustrate the clinical background of orders and results (see Chapter 5$)$.

In an electronic medical-record system it is possible to combine these different organisations. Together, they form the searching structure of the medical record. Elementary paragraphs contain information that is not further divided into indexable sections. The level of detail to which these elementary paragraphs are specified is called the granularity of the medical-record structure. In general, one paragraph corresponds with one observation or intervention. In the narrative parts of the medical record, however, this needs not be the case. Medical history and physical examination may be presented (1) without partitioning, ${ }^{13-15}$ (2) with paragraphs on the level of organ systems, ${ }^{4,16}$ and (3) partitioned into separate observations or even single findings. ${ }^{15.17 .18}$ Progress notes may be presented are presented (a) undivided ${ }^{16}$ and (b) partitioned into problem-sections. ${ }^{19}$ Apparently, the granularity of medical narratives is an arbitrary feature without existing consensus.

As explained before, the retrieval of information from the medical record can be divided into two steps: searching relevant paragraphs and reading their content. The ease of finding a specific paragraph is dependent on their number: the more paragraphs, the more complex the searching structure, and the more difficult to locate the relevant paragraph. The ease of reading the paragraph is dependent on its size: the smaller the paragraph, the more specific the information it contains, and the easier to find the information that is needed. Regarding a specific patient case, the number of paragraphs is inversely proportional to their size. Consequently, facilitation of searching may have an opposite effect on the ease of reading and vice versa.

Our ain was to develop an electronic medical-record system that would (1) provide the adwantage of a searching structure in which different organising principles are combined and (2) accommodate different gramularities within the narrative parts, which would enable us to find the best trade-off between searching and reading. 


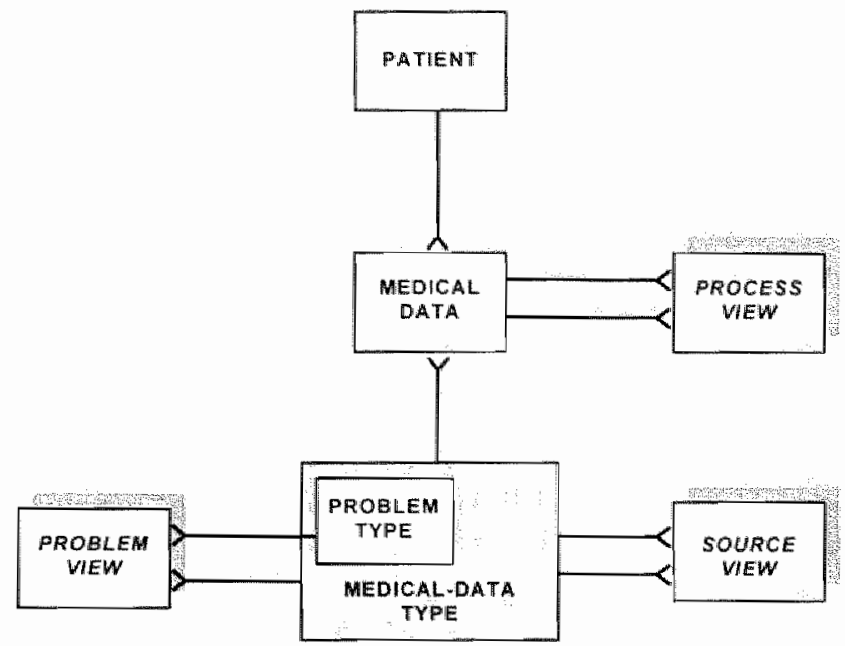

Figure 6-1:

Conceptual data model of the structure of the medical record. The different views of the searching structure are shadowed. The terminology base is in gray.

Symbols and lines are according to the entity-relationship-diagramming technique.

\subsubsection{Facilitation of reading: the presentation format of medical narratives}

The most vivid discussion about medical narratives in EMR-systems, at the moment, concerns the choice between the expressive power of free text ${ }^{20.27}$ and the unambiguous understandability of controlled expressions. ${ }^{22,23}$ This discussion involves the entry and storage of medical narratives, but not so much their presentation. We encountered only two pure representatives of the time-oriented medical record that present narrative information in coded format (which easily fits in a two-dimensional flowsheet). ${ }^{17,24-26}$ On the other hand, many systems that store medical narratives in coded format utilise, for presentation purposes, a natural-language generator to convert these codes into natural prose. ${ }^{58-12}$ Hence, for the presentation of medical narratives, natural prose seems the de facto standard. We had no reason not to use free text for presenting medical narratives.

\subsubsection{The user-interface: simple and stable screen design}

Although data entry and information retrieval each have their own requirements, for the design of the user-interface of the electronic medical-record system they must be considered as two sides of the same coin: in daily medical practice, reading and writing the medical record often go together. In two studies considerable attention has been paid to the design of the user interface in order to facilitate, respectively, data entry and information retrieval of medical narratives. ${ }^{4.5}$ Both studies emphasised the users" preference for visual cues to enable a reading strategy of pattern recognition when consulting the medical record. Such a strategy allows the reader to see in a glance, whether or not a certain place on the screen is filled with information. To provide visual cues, the user-interface should support paging rather than scrolling panels, static rather than dynamic (context-sensitive) menus and forms, and display text linearly 


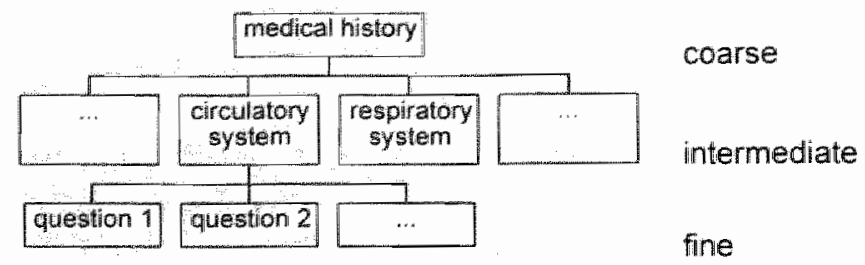

Figure 6-2:

Granularity of medical narratives as derived from their position in the source view. The dotted lines indicate the bottom level of the coarse, intermediate, and fine granularity.

structured rather than with hypertext-links. According to these insights, we reduced the complexily of the user-interface and strived for a uniform layout of screens.

\subsubsection{Use for research}

Our second goal was to use the system for research purposes. The system had to guide potential users (practising physicians) through a specific test protocol, record the users" searching process, and carry out an evaluative questionnaire at the end. The specific study to which we refer here was a comparison of speed, completeness, and user appreciation when retrieving information from medical narratives with different granularities.

\subsection{System description}

The EMR-system we have developed consists of (1) a database in which both structure and content of the medical record are recorded, (2) a user-interface with three different presentation windows, (3) a number of programming modules with functions for accessing the database via the user-interface, and (4) a special research module to conduct studies concerning the use of the medical record. The system was implemented in an MS-Windows-3.11 $\mathrm{TM}$ environment. We used a Btrieve 5.0 database, Visual-Basic $3.0^{\mathrm{rm}}$ for developing the user-interface, and a combination of Visual-Basic procedures and MS-Windows-DLLs to make the medical-recond system functioning.

\subsection{The patient database}

The database underlying our EMR-system is the repository for storing (1) the types of paragraphs, i.e., the kinds of medical data we distinguish, (2) the content of these paragraphs, i.e., the patient data that have been recorded, and (3) the searching structure of the medical record, i.e., the different views on the medical record (Figure $6-1$

1. The types of paragraphs we distinguish are all stored in one table MEDICAL-DATA TYPE. This table contains the names of observations, interventions, laboratory tests, etc. With respect to medical narratives, the names of all possible paragraphs types are stored, from the very broad medical history, via the intermediate circulatory system, to the very specific paragraph type palpitation of the heart. Also the names 


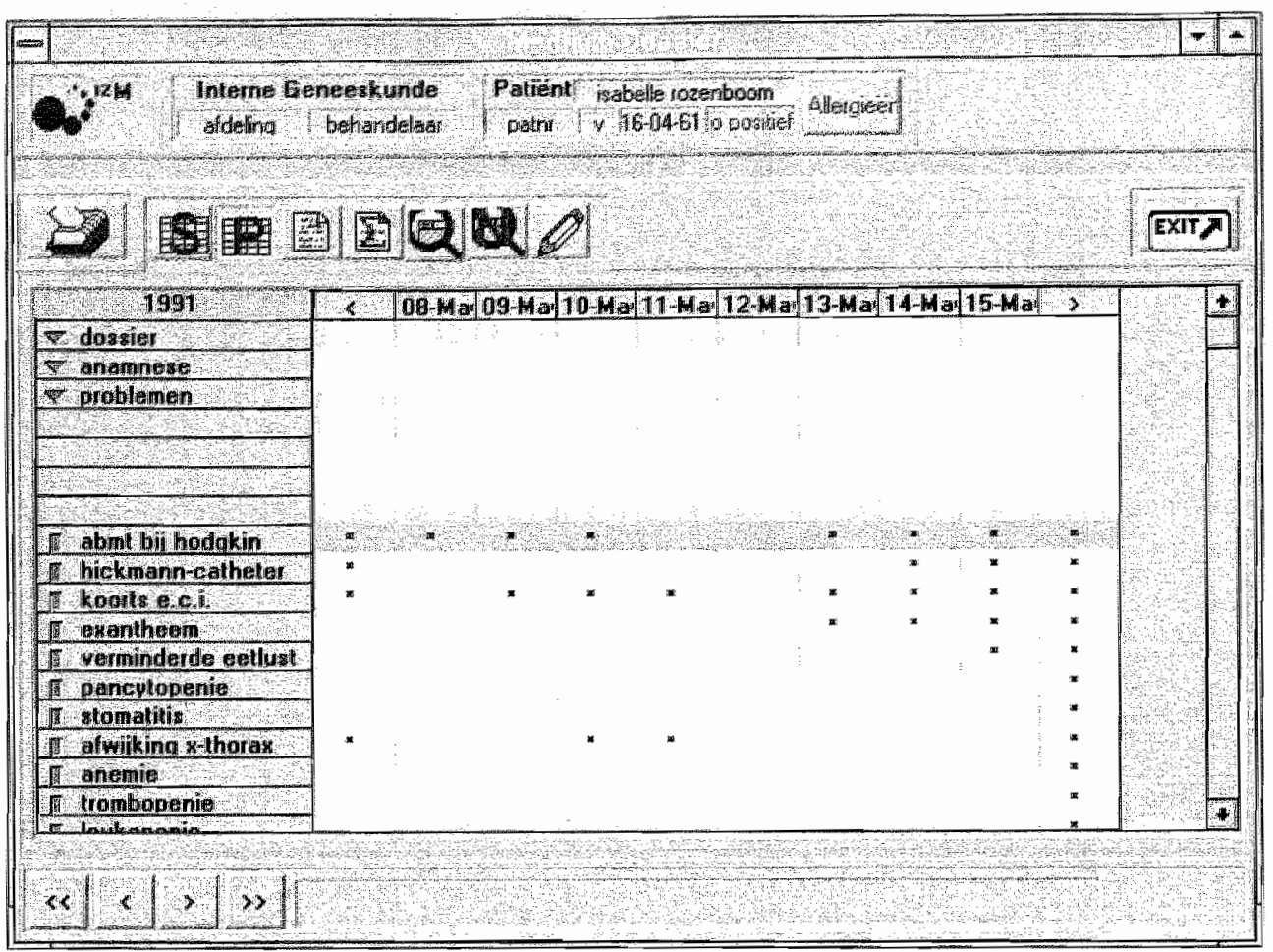

Figure $6-4$.

Overview window-Flowsheet of problem-partitioned progress noles.

Top level with problem list and correspanding progress notes. In this example, the problem "autologous bone-marrow tramsplantation" ["abmt bij hodg|kin"] is selected over the entire time range. From this point on, the user can access the selected progress notes for reading or jump to the lower level of the flowsheet, which will show a list of the problem-relevant paragraphs.

place where process links between individual paragraphs (medical data) can be stored as described in 6.2 .1 .

The granularify of medical narratives, i.e., the elementary paragraph types that are not further subdivided, cannot be inferred from Figure 6-1. It is deduced from which types of paragraphs in the table MEDICAL-DATA TYPE are on the bottom of the sourceoriented hietarchy defined in the table SOURCE VIEW. Only paragraph types on the botton of this hierarchy are used for recording patient information (see Figure 6-2).

\subsubsection{The user interface}

The user interface consists of a fixed-size working screen comprising two information panels and two toolbars. The upper information panel (Figure 6-3: A) presents the information that can usually be found on the cover of a paper-based medical record: identifying information about the patient, the department and the responsible physician, and clinical information that must be directly accessible in emergency situations, like blood group, allergies, etc. The upper toolbar (Figure 6-3: B) contains buttons for selecting a patient and one of the lower information panels, and for exiting the program. For each different information panel, this toolbar is extended with buttons 


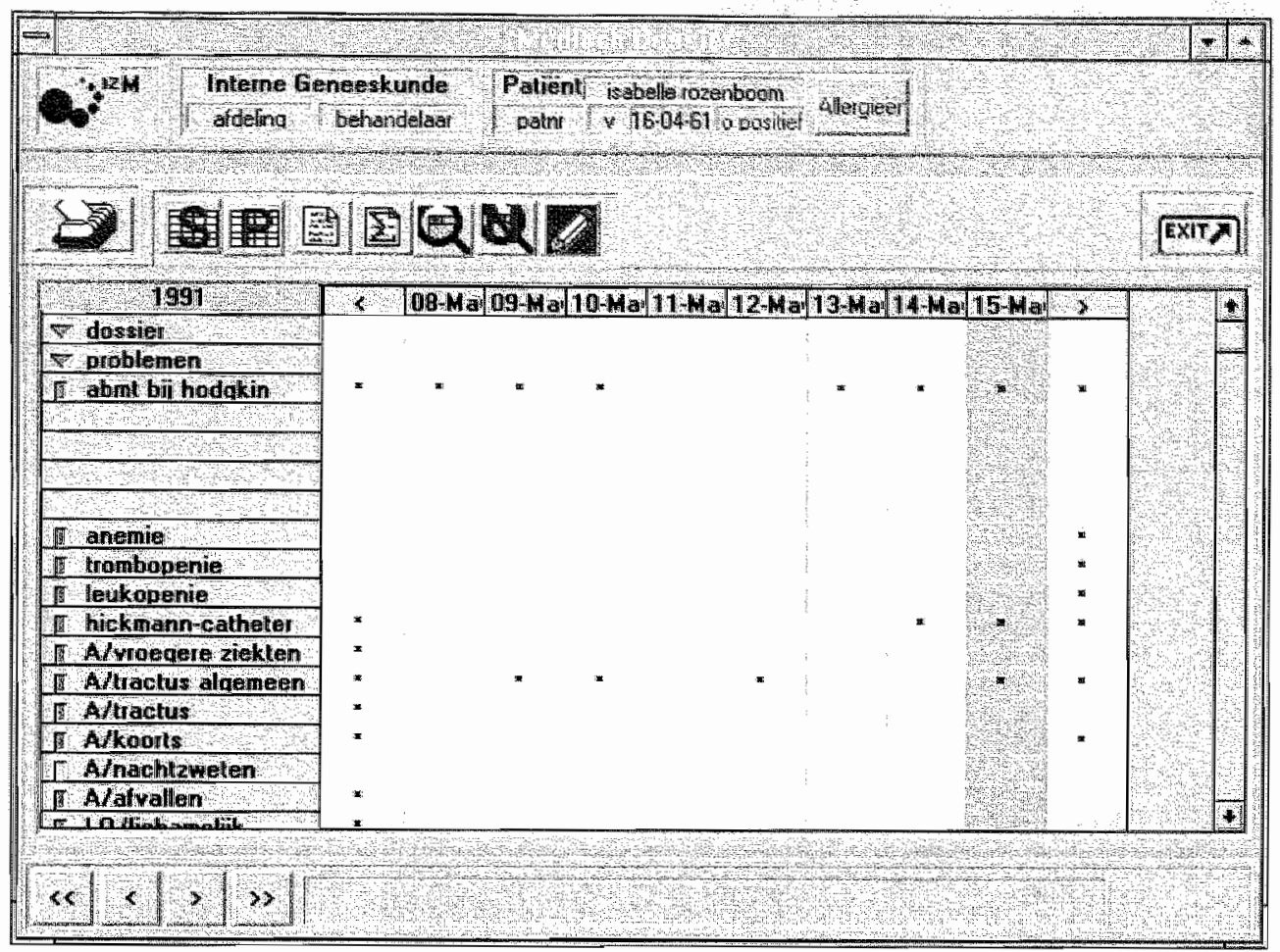

Figure 6-5:

Overview window-Problem-oriented flowsheet of the problem "autologous bone-marrow transplantation " [abmt bij hodgkin]. On the vertical axis, all types of paragraphs are presented that may contain relevant information with respect to this problem. The highlighted column covers all information about the problem recorded on May 15th, i.e, the progress note (in the stacky together with possibly relevant observations of that day.

that provide specific functions for manipulating that window. The lower, large, information panel (Figure 6-3: C) presents the structure and content of the medical record. This panel can take the shape of an overview window, a reading window, and an edit window. The lower toolbar (Figure 6-3: D) provides buttons to navigate through time.

\section{The overview window}

Each consultation of the medical record starts from the overview window, which is shaped as a two-dimensional matrix (Figure 6-3 - Figure 6-5). The vertical axis displays either the source view or the problem view, the horizontal axis represents the time view (per default in days). Cells with an asterisk indicate paragraphs that contain information. The first $("<)$ and last $\left({ }^{*}>{ }^{*}\right)$ column contain all data recorded before and after the period presented on the horizontal axis. The user can change this period with the buttons on the lower toolbar. Dependent on which view is presented on the vertical axis, a flowsheets is source-oriented or problem-oriented. The user can toggle between source-oriented and problem-oriented view by the use of icons on the toolbar. 


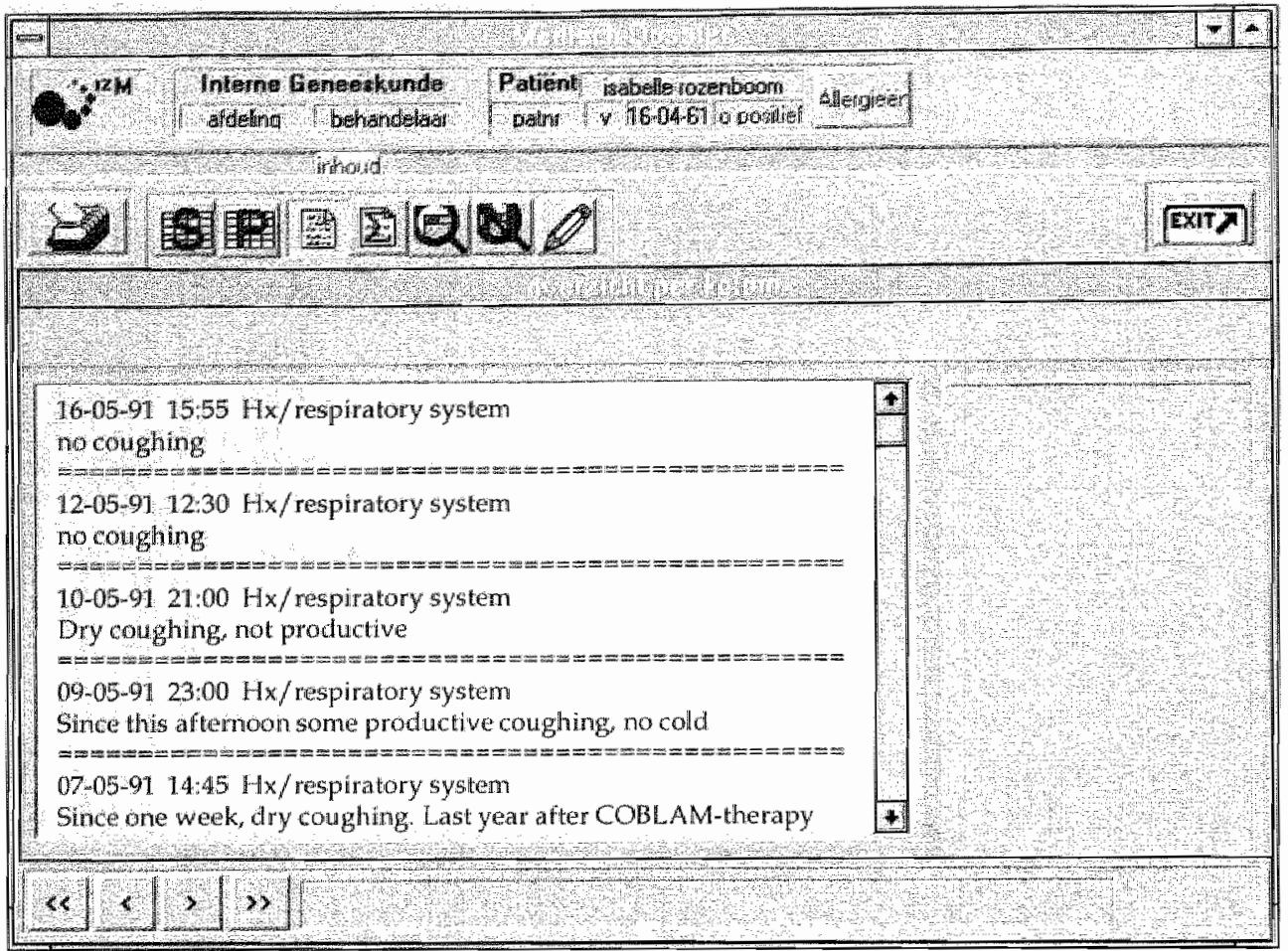

Figure 6-6:

Reading wholow-Collation of paragraphs, in descending time order, containing medical history information concerning the respiratory system. The content of the reacling window is translated from Dutch.

- The source-oriented flowsheet - In this flowsheet, the user can browse through the hierarchical organisation of paragraph types. Types lower in the hierarchy can be displayed or hidden by a simple mouse click on the name of the parent type (right column, in grey). For example, clicking on medical history [anamnese] will show a list of organ systems (Figure 6-3, starting from the eighth row). Clicking, subsequently, on digestive system [digestivus] will show a list of separate questions that can be asked about this system. The path the user has followed is stored in a stack that can be traced back whenever needed. The stack is presented in Figure 6-3 starting from the first row.

- The problem-oriented flowsheet - This flowsheet shows, on the vertical axis, the patient's problem list and presents the progress notes in the cells next to the corresponding problems (Figure 6-4). Clicking on one of the a problems will show a list of items (paragraph types) that may be relevant to the given problem (Figure 6-5). The user can toggle between source-oriented and problem-oriented view by the use of icons on the toolbar. 


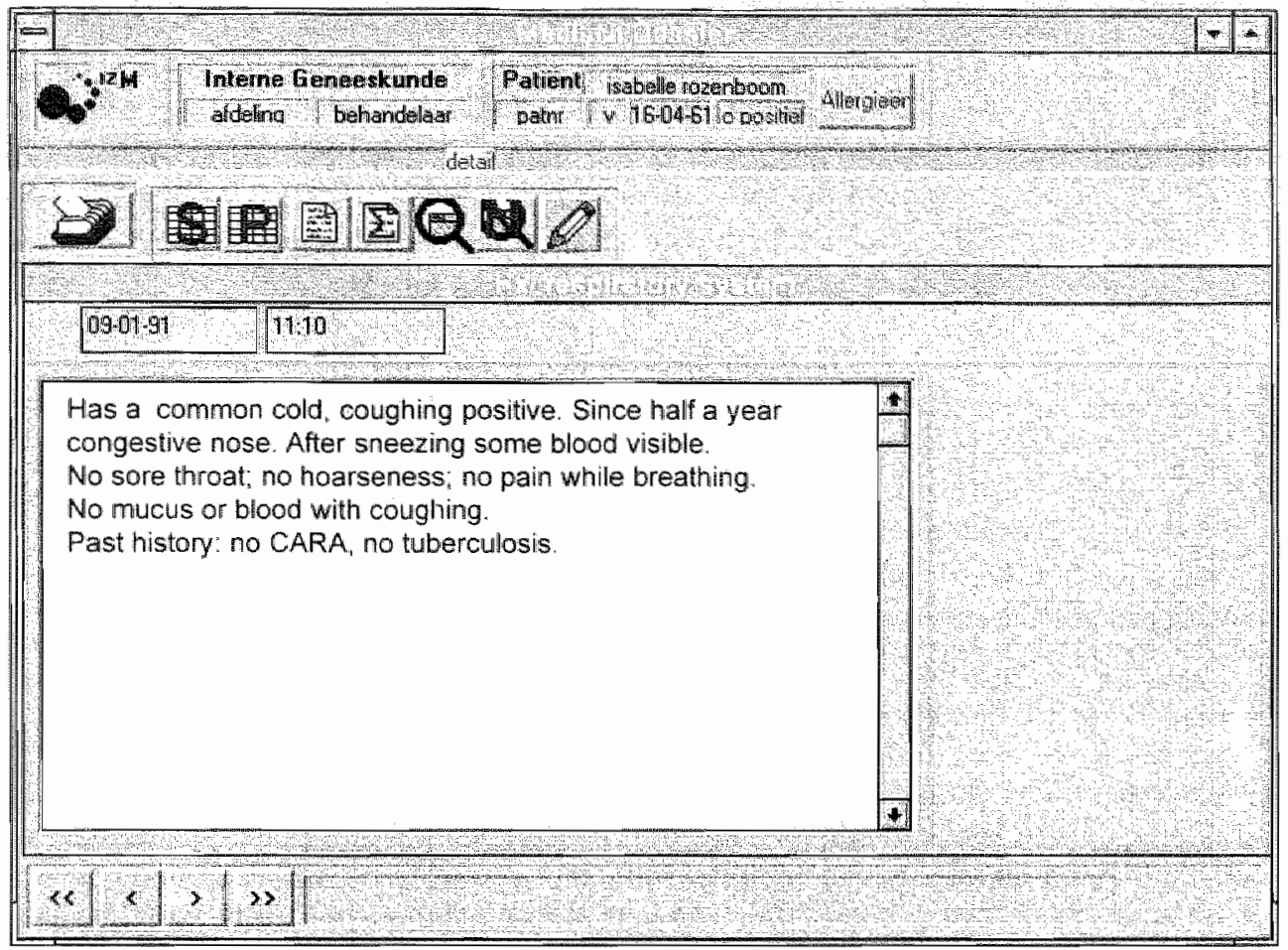

Figure 6-7:

Edit window-Content of one paragraph, consisting of medicall history information concerning the respiratory tract, recorded at January 9 thi, $11.10 \mathrm{AM}$. The content of the edit window is Iranslated from Dutich

\section{The reading window}

In the overview window, the user can select one or more paragraphs for viewing their content. Any rectangle of cells can be selected. A vertical cross-section of the matrix results in a description of the present state of the selected period (Figure 6-4). A horizontal cross-section results in a description of the evolution in time of the sellected type of paragraph (Figure 6-5). The content of the selected paragraph is then presented in a reading window, in free-text format, preceded by a paragraph heading denoting type of paragraph and time of recording (Figure 6-6). In case more paragraphs are selected, their contents are collated. The user can toggle between ascending and descending time order by the use of icons on the toolbar. The default presentation is in descending time order. The reading window can contain up to $64 \mathrm{kB}$ of text, which corresponds with the content of nearly fourteen plain-text A4-documents.

\section{The edit window and the process view}

The edit window is accessible from the presentation window, but also directly from the overview window. It contains only one paragraph at a time (Figure 6-7). Its function is twofold: (1) to add a new paragraph or to modify or delete existing paragraphs, (2) to get access to the process view, for generating or consulting process links. 


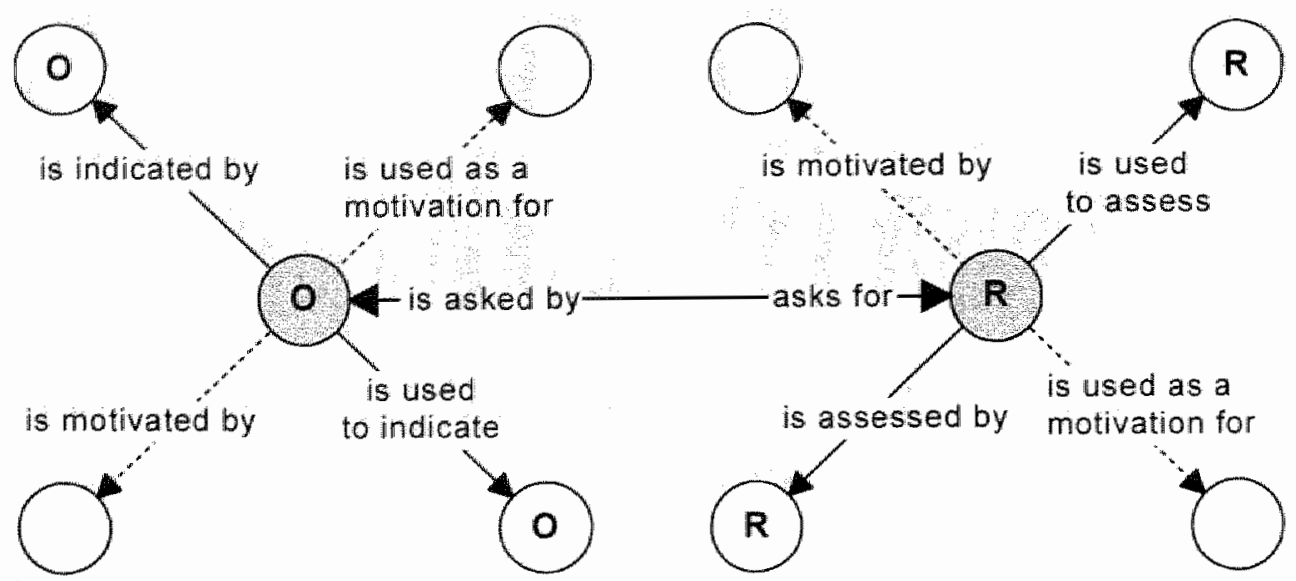

Figure 6-8:

A current paragraph (in grey, order or result, respectively) represented as a junction of process links: Each link should be read in the direction of the arrow. The link between both current paragraphs is the completion link. The other process links are indicated by their names.

$O=$ order; $R=$ result. Time flows from left to right.

To better understand the construction of the process wiew and the role of a specific paragraph in it, it one should consider a current paragraph as a potential junction of process links (Figure 6-8). Paragraphs with the character of an order may have a completion link in forward direction and indication links both forward and backward. Paragraphs with the character of a result have a completion link in backward direction and assessment links both forward and backward. Orders and results may have motivation links both forward and backward. More details about these links are given in Chapter 5.

\subsubsection{The research module: a "guided tour" through parient cases}

Our system is used as an instrument for research with respect to specific aspects of the medical record. For implementing the research module, we used the idea of a "guided tour" in which the user is guided through a test protocol consisting of a sequence of instructions and exercises, with the possibility to give evaluative feedback at the end. Any kind of research concerning the use of information from the medical record can be supported, as long as the test protocol fits the idea of a "guided tour". We used the system for studying the ease of information retrieval, but it can also be used, for example, to study the ratio behind medical decisions in the context of medical audit and medical education. The instructions and exercises can be defined at will and are stored in the research database before the test starts.

\section{Instructions}

The system can automatically present a series of medical records to the user, selected from a number of patients and medical-record types, for each user in a pre-defined order. Each patient case is accompanied by a number of questions that are presented one by one. Each question prompts the user to perform an exercise, e.g., to obtain evidence for establishing a differential diagnosis. An exercise has two time-stamps: 
one related to the patient case (the case-time), and one related to the execution of the exercise itself (the exercise-time). A question is presented in a separate questionwindow. The left side of this window shows the question itself (with some additional information if needed), while the right side is reserved for presenting the results of the corresponding exercise. During an exercise, the user can always return to the question window to re-examine the question and the already obtained results.

\section{Exercises}

During an exercise, the user has to consult the medical record to obtain the required evidence and he can add some comments this answer if needed. The user can browse freely through the medical record of the patient concerned. The only restriction is that the user cannot access information recorded later than the case-time. All user-driven events of the system during the exercise are recorded in a research database, including the time they took place. The following kinds of actions are distinguished:

1. Searching - The user can navigate freely through the medical record. He can toggle between the different overview windows, display and hide sub-levels in these windows, select paragraphs, open the reading window, etc. All these events are logged, to enable a play-back if needed.

2. Tagging evidence - With the mouse, the user can mark a text phrase and subsequently confirm its relevance by pusthing a specific evidence button on the toolbar. The selected phrases are stored, together with the type and time-stamp of the paragraph they have been extracted from.

3. Finishing the exercise - The user can type an answer to the question by adding some comments to the obtained evidence. Finally, the user can indicate that he has finished the exercise by pushing a specific complete button in the question-window.

\section{Evaluation}

After the user has finished the exercise, the system will present the next question or, after having finished the last question, the next patient case. At the end of the "guided tour", and also between the different patient cases, some evaluative questions can be presented in a separate evaluation window. At the moment, this window has been developed for closed answers only. The number of possible questions is in finite.

\section{The research database}

The research module contains a separate database in which the instructions for and results of the exercises are stored. As opposed to the electronic medical-record's own database, the research database has been implemented in MS-Access ${ }^{\mathrm{T}}$, in order to make its content better accessible for analysis. The structure of the research database is given in Figure 6-9. In the database the following information can be stored:

1. Instructions to the test person - The research database includes the sequence in which specific medical-record types and patient cases are allocated to the different users in the order prescribed by the test design. This sequence is stored in the table TEST ORDER. The exercises that will be offered to the test persons are stored in the table EXERCISE, in a fixed order for each patient case. 


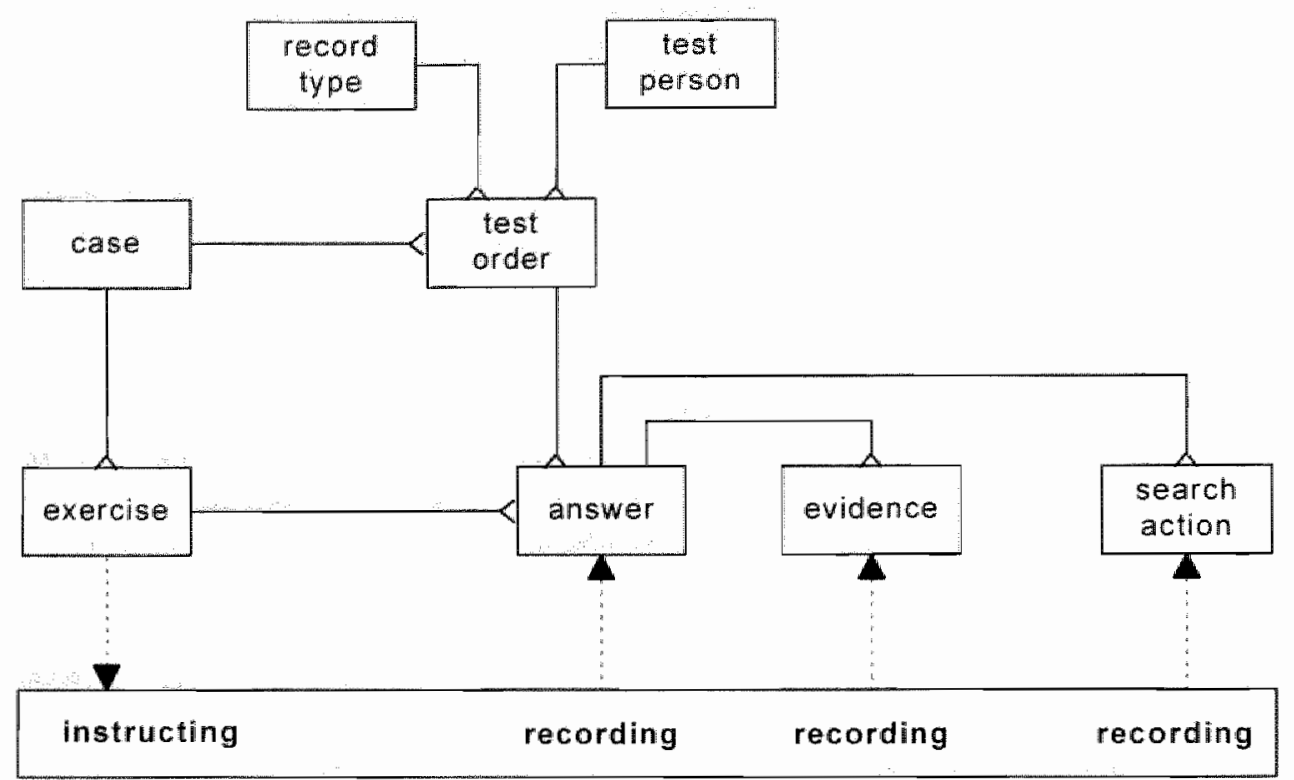

Figure 6-9:

Conceptual data model of the research database. The dotted arrows are interactions with the user. Symbols and lines are according to the entity-relationship-diagramming technique.

2. Recordings of user-driven events - How the test persons navigate through the system is recorded in the table SEARCH ACTION. The given answer and the time of completion of an exercise are recorded in the table ANSWER, while the evidence marked for the answer is recorded in the table EVIDENCE.

\subsection{Using the system as a research tool}

The electronic medical-record system was in this case used to investigate with which granularity medical narratives can best be presented to optimally support the retrieval of information for medical practice, in particular for inpatient medical care. For this reason, we defined the following types of medical records, which differed in the granularity with which medical narratives were presented.

1. A coarse type, which resembles the traditional medical record, presenting medical history, physical examination, and progress notes as undivided paragraphs.

2. An intermediate type, in which medical history and physical examination are divided into organ-system and progress notes are partitioned into problem sections.

3. A fine type, in which medical history and physical examination are divided into single questions and observations, and progress notes are, again, partitioned into problem sections.

We used three outcome measures: speed of searching, completeness of searching, and user appreciation. The study of speed and completeness will be reported in Chapter 7 . Here, we give a short description of the study design and of the adaptation of the 
EMR-system needed for conducting this study. We also present the results concerning the outcome measure of user appreciation.

\subsection{Shudy design}

The study was designed as a trial comparing the searching performance of physicians. when using three different medical-record types as were described above. Thirty-six randomly selected test-persons (specialists and residents from the domain of internal medicine) were asked to consult our EMR-system three times, each time using a different record type. The medical record comprised three real patient cases, each stored in all three medical-record types. We chose a very demanding setting, namely a selection of voluminous and complex inpatient cases in internal medicine. For each case, several situations for consultation were formulated in which the test persons had to answer one or more pre-defined questions concerning the patient and his treatment.

Statistical analysis was conducted with SPSS-for-Windows-6.0 $\mathrm{rm}$, to test whether significant differences in speed, completeness, and user appreciation could be demonstrated. To balance for differences caused by inter-subject variations and other effects (e.g., patient case and series effect) we used a balanced incomplete block design for three "treaments". Further details about this design will be given in Chapter 7.

\subsubsection{Preparation of the study}

As a preparation of the study the three selected patient cases were entered into the database. Narrative data were transcribed from the original hand-written documents of the paper medical record. Test results from auxiliary services, such as laboratory, pathology, and X-ray, were automatically downloaded from the BAZIS hospitalinformation system as it is used in the Maastricht University Hospital. ${ }^{27}$ We developed a conversion module to obtain the desired format for the Btrieve-database.

For our specific research purpose, we faced the problem that all test persons would use the system only once, so the system had to be, above all, quick and easy to learn. As it was not reasonable to ask more than one hour from our test persons, the exercises hors concourse could not take more than about 10 to 15 minutes. Hence, we reduced the complexity of the user interface by hiding elements that were not necessary for the test. All standard window-manipulating functions were hidden. Pull-down menus, though also very familiar for experienced MS-Windows users, were disabled. All functions needed for the test could be activated by icon buttons presented on the toolbar. The process view, although potentially a very useful perspective, was disabled because it could not be reconstructed from the selected cases and we did not want this view to interfere with the test persons' view on the process that would lead to bis answer. Since the test persons were neither allowed to modify medical clata, the added value of the edit window became marginal, hence it was decided to hide it.

\subsubsection{Evaluation results}

We needed thirty-six physicians to reach a balanced set of outcomes. It was decided to take 24 specialists and 12 residents. In total $80 \%$ of the approached specialists and $73 \%$ of the approached residents accepted to participate in the study. Non-co-operation 


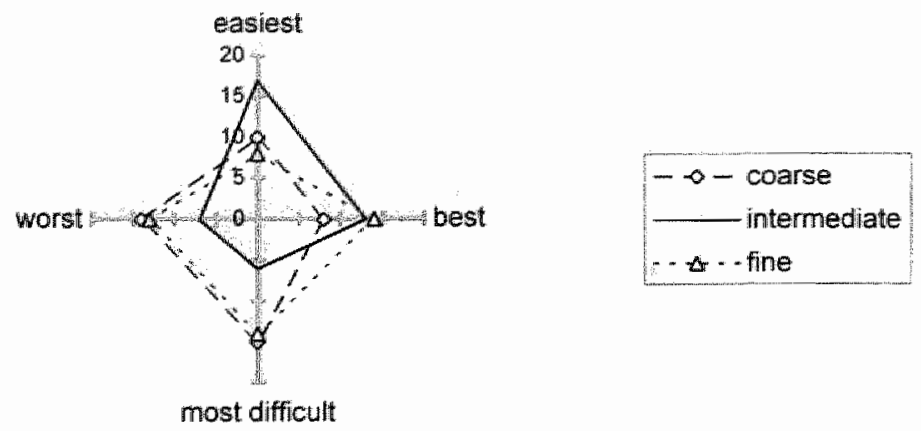

Figure $6-10$

Radar diagram showing insight in the patient case on the horizontal axis and ease of searching on the vertical axis. Both axes have the same scale representing the number of answers $(n=36)$. The right-upper quadrant is the best combination of both.

was, in all cases, due to lack of time. The average duration of the entire test, including explanation and preparatory exercise, was 50 (38-68) minutes, of which 44 (32-63) minutes were spent on the proper test. On average, residents performed the test in 42 minutes, specialists in 45 minutes. All questions for searching were answered satisfactorily, according to the test persons. To get an impression of their experience with computers, we asked the participants some questions in advance. From the 36 participants, 23 used the computer daily, 8 from time to time, and 3 seldom; 4 test persons did not answer this question. Only 2 persons qualified themselves as experienced computer user, 18 as moderately experienced users, 14 as novice, with also 4 answers missing.

To compare their appreciation of the different medical-record types, we asked the test persons, at the end of the test, to indicate which record type, for searching purposes, they considered easiest and most difficult, respectively. We also asked which record type provided the best and worst insight in the patient case, respectively. The results of these questions are presented in Figure 6-10. The diagram suggests that the intermediate structure is appreciated best for providing a combination of easy searching and proper insight in the patient case. The sample was too small, however, to demonstrate any significant differences.

The results concerning the speed and completeness of information retrieval are reported in Chapter 7 . There, we will conclude that searching was fastest when using the medical-record type with intermediate granularity, although not significantly different from the medical-record type with fine granularity. Searching when using the medical-record type with coarse granularity, however, was significantly slower than both other (paired t-test, $\mathrm{p}<0.01$ ). We found no difference in completeness of retrieved information: this amounted to $80 \%$ of the required information for each medicalrecord type. 


\subsection{Discussion}

The use of a guiding structure to support the retrieval of information is not new. Around 1970, the introduction of the problem-oriented and time-oriented medical records brought this aspect under the attention of a broad medical audience ${ }^{62}$ With the first publications about electronic medical-record systems, however, other more technical issues began to dominate the discussion about medical-record keeping (such as system architecture, input devices, and the use of standards). In the mean time, source-oriented and time-oriented flowsheets were actually implemented (more or less) in several classical electronic medical-record systems. ${ }^{29}$ Medical narratives were usually not included in such flowsheets, or only in coded format.

What makes our system unique is the presentation of free-text medical narratives in flowsheets, albeit not for direct reading. Yet, the user is able to observe the existence of narrative information about present state and time trends in one glance. By opening a selection window, the actual content of this information is presented. Compared to the inclusion of coded narrative data in flowsheets, our approach necessitates an additional action, but enables the use of all nuances of natural language.

\subsubsection{Presentation structure and storage format}

Among existing electronic medical-record systems, medical narratives are presented in diverging granularities (Paragraph 6.2.1). The evaluation reported in this chapter suggests that a division into organ systems and problem-sections is appreciated best. In Chapter 7 we demonstrate that this granularity also allows the fastest searching. However, it should be noted that in our study we only used voluminous patient cases. For smaller cases it is possible that the granularity of medical narratives will be less critical for the retrieval of information.

In the prototype system presented in this chapter, medical narratives are stored as free text in the same granularity as we present them. We made this choice for reasons of convenience. Technically, it is possible to use different granularities for data entry, data storage, and data presentation. When narratives are entered and stored as plain free text, they must first be interpreted before they can be divided into paragraphs. Systems for the interpretation of natural language will probably play a major role in the future. ${ }^{1930.31}$ At the moment, however, most experience with natural-language interpretation is obtained with discharge summaries in some well-described domains. ${ }^{32 .}$ 34

In case medical narratives are entered and stored as separate observations, they must be collated together and converted to fluent prose before they can be presented as free-text paragraphs of organ systems. Experience with this so-called generation of natural language from structured (or even coded data) is more widespread and the domain of application is broader. ${ }^{5.8 m}$ Recently, we also implemented a (provisional) function that collates information from detailed paragraphs into less detailed paragraphs when presenting them for reading. 


\subsubsection{Problems with the procesg view}

The avallability of a process view on medical data will make the medical-care process more transparent. It may open new perspectives for medical audit and education: physicians can review on which evidence they take decisions, medical students can review how physicians take decisions, and teachers can review how medical students take decisions. But also for direct medical practice the process view may be useful.

The idea of a process view that enables the physician to uncover relevant relations between medical data has been also described by others. ${ }^{35.36}$ Barrows et al admitted that they encountered "logistic problems with the interface" when trying to implement the process view. We also had the experience that inclusion of the process view makes the user-interface much more complex. The implementation we chose works, but is difficult to explain to potential users. As a major drawback we consider the fact that physicians are not yet familiar with the idea of the process view. However, the need for a medical record that reflects the process of medical practice more closely than before is acknowledged widely. ${ }^{19}$ We believe that this need will grow further and eventually force the physician to record his deliberations more explicitly. Hence, a major challenge for the near future is to develop an intuitive user interface for handling the process view.

\section{5 .3 Limitations of the humancomputer interface}

In our system we have implemented most facilitating measures for reading that were suggested in the literature. 4.5 Our electronic medical-record system has a simple and stable user-interface, with all medical data accessible via the same way. All windows are presented at a fixed position on the screen and have a fixed width. The userinterface contains many fixed-position cues, and the flowsheets always provide a complete set of medical-data types, regardless of whether they were used or not.

One feature of the user-interface, however, is not according to these measures: our system does not support paging through windows or panels. The software-development kit we used (Visual Basic $3.0^{\mathrm{rm}}$ ) only supports scrolling, which does not provide fixedposition eues. The text-window object provided by Visual Basic 3.0 Tr has also some other limitations. One text window can only present one format of text at a time, which impedes the use of different layouts. For example. it is not possible to provide more visual cues by presenting paragraph headings in bold, time-stamps in italic, and the content of medical narratives in normal font.

In the text windows we used it is neither possible to search by keywords. Free-text searching by keywords (by the use of so-called search engines) has become popular with the growing use of World-Wide-Web browsers. For searching in medical natratives with coarse granularity it will certainly have an added value. On the other hand, the added value of search engines should not be overestimated. When reading medical narratives, also the clinical context of medical data has a guiding role. Search engines do not take this context into account, which may lead to an erratic way of searching.

The system is presently implemented on a stand-alone 486-PC-configuration with a VGA-resolution screen. This resolution restricts the number of rows and columns on the screen, which makes the retrieval of information more dependent on scrolling. For 
example, it is not possible to present all organ systems of the medical history together on one screen. We expect that the ease of information retrieval will benefit much from a higher-resolution screen.

\subsection{Future plans}

In the discussion we mentioned several desired functionalities that have not yet (or only provisionally) been implemented: a paging function for windows and panels, textprocessing functions to facilitate reading, the use of a high-resolution screen to display more information at a time, and a user-interface that properly supports the process view. Although we plan to implement these functions in the future, the prototype system has fulfilled its goal. At the moment we face the next big challenge: upgrading the system to a true medical workstation with on-line connection to the hospitalinformation system. The integration of the medical record with the other systems under study at our department (voice input, electronic consultation of practice guidelines and literature databases) would be much facilitated by the use of a common medical terminology and by co-operation with one or more commercial vendors of electronic medical-record systems. For the prototype system, we imagine a possible future role in medical education. It may be useful for training and testing case-based reasoning of medical students, because teachers will be able to monitor the role of evidential information in the decisions the students take.

\subsection{References}

1. de Bruyn B, Verheijen LM, wan Nes FL, Arends JW. Assigning SNOMED codes to natural language pathology reports. Medical Informatics Europe. Vol. 34-A, 1996:198-202.

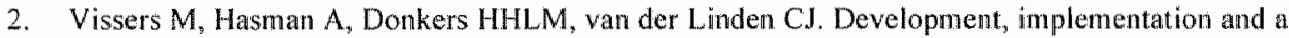
first evaluation of a protocol processing system (ProtoVIEW). Comp Meth Prog Biomed 1995; $47: 81-92$

3. Wiesman F, Hasman A. A graphical user interface for biomedical literature search. Medical Informatics Europe. Vol. 34-A, 1996:624-628.

4. Nygren $E$, Johnson M, Henriksson P. Reading the medical record. II. Design of a humancomputer interface for basic reading of computerized medical records. Compur Methods Programs Biomed 1992; 39:13-25.

5. Poon AD, Fagan LM, Shortliffe EH. The PEN-Iwory project: Exploring user-interface design for the selection of items from large controlled vocabularies in medicine. J Anu Med Inform Assoc 1996; 3:168-183.

6. Fries JF. Alternatives in medical record formats. Med Care 1974; 12:871-81.

7. McDonald CJ. Computer-stored medical record systems [editoriall. MD Comput 1988; 5:4-5.

8. McDonald $\mathrm{CJ}$, Tierney WM, Blevins $\mathrm{L}$. The benifits of automated medical record systems for ambulatory care. In: Orthner $\mathrm{HF}$, Blum $\mathrm{BI}$, eds. Implementing health care information systems. New York: Springer-Verlag, 1988.

9. Stead WW, Hammond WE. Computer-based medical records: the centerpiece of TMR. MD Comput 1988; 5:48-62.

10. Trace D, Naeymi Rad F, Haines D, et al. Intelligent Medical Record-mentry (IMR.E). J Med Syst $1993 ; 17: 139-51$.

11. Gouveia Oliveira $A_{y}$ Raposo VD, Azevedo AP, et al. SISCOPE: a multiuser information system for gastrointestinal endoscopy. Endoscopy 1991; 23:272-7. 
12. Bernater I. Concepttal graphs as an operational model for descriptive findings. Proc Annu Symp Comput Appl Med Care 1991:214-8.

13. Stead WW, Hammond WE, Straube MJ. A chartless record--is it adequate? J Med Syst 1983 ; $7: 103-9$

14. Safran C, Ponter D, Lightfoot J, et all. ClinQuery: a system for online searching of data in a teaching hospital. Ann Inten Med 1989; $111.751-6$.

15. van Ginneken AM, Stam H. Can one patient record accommodate the diversity of specialized care? Proc Annu Symp Comput Appl Med Care 1995:406-10.

16. Poon AD, Fagan LM. PEN-Ivory the design and evaluation of a pen-based computer system for structured data entry. Proc Annu Symp Comput Appl Med Care 1994:447-51.

17. Bolens $M$, Borst $F$, Scherrer JR. Organizing the clinical data in the medical record. MD Comput $1992 ; 9: 149-55$.

18. Heathfield HA, Hardiker $\mathrm{N}$, Kirby J, Tallis R, Gonsalkarale M. The PEN \& PAD medical record model: development of a nursing record for hospital-based care of the elderly. Methods Inf Med $1994 ; 33: 464-72$.

19. Dick RS, Stein EB. The computer-based patient record - an essential technology for health care. Washington DC: National Academy Press, 1991.

20. Blois MS. Information and medicine - the nature of medical dscriptions. Berkeley, CA: Unversity of Califomia Press, 1984.

21. Essin DJ. Intelligent processing of loosely structured documents as a strategy for organizing ellectronic health care records. Methods Inf Med 1993; 32:265-8.

22. Rector AL. Marking up is not enotugh [editorial]. Methods Inf Med 1993; 32:272-3.

23. Barnett $\mathrm{GO}$, Jenders $\mathrm{RA}$, Chueh $\mathrm{HC}$. The computer-based elinical record-where do we stand? [editorial]. Ann Intern Med 1993; 1 19:1046-8.

24. Fries JF. Time-oriented patient records and a computer databank. Jama 1972;222:1536-42.

25. Fries JF, McShane DI. ARAMIS (the American Rheumatism Association Medical Information System). A prototypical national chronic-disease data bank. West J Med 1986; 145:798 804.

26. Borst F, Bolens M, Scherrer JR. A fully interactive fully coded medical record for the pediatrician: towards a source of medical knowledge originating from the practitioner, Proc Medinfo' 92 , 1992. Vol. 751-754. North-Holland.

27. Bakker AR. An integrated hospital information system in The Netherlands. MD Comput 1990 ; 7:91.97.

28. Weed LL. Medical records that guide and teach. N Engl J Med 1968; 278:593-600,652-657.

29. McDonald CJ, Barnett GO. Medical-record systems. In: Shortliffe EH, Perreault LE, eds. Medical Informatics - Computer Applications in Health Care. Reading MA: Addison-Wesley, 1990:181-218.

30. Kay S. Purves IN. Medical records and other stories: a narratological framework. Methods Inf Med 1996; 35:72-87.

31. McDonald CJ, Tierney WM. Computer-stored medical records. Their future role in medical practice. Jama 1988:259:3433-40.

32. Lin $R_{\text {, Lenert }} \mathbb{L}$, Middleton $B$, Shiffman $S$. A free-text processing system to capture physical findings: Canonical Phrase Identification System (CAPIS). Proc Annu Symp Comput Appl Med Care 1991:843-7.

33. Sager $N$, Lyman M. Tick LI, Ngô TN, Bucknall CE. Natural language processing of astloma discharge summaries for the monitoring of patient care. Proc Annu Symp Comput Appl Med Care 1993:265-268.

34. Sager $N$, Lyman $M$, Nhan $N T$, et al. Clinical knowledge bases from natural language patient documents, Proc Medinfo'92, 1992. North-Holland.

35. Barrows $\mathrm{RC}$, Ir., Jothnsom SB. A data model that captures clinical reasoning about patient problems. Proe Annu Symp Comput Appl Med Care 1995:402-5.

36. van Gïmeken AM, Stam H, Moorman PW. A multi-strategy approach for medical records of specialists, Proc Medinfo'95, 1995. North-Holland. 


\title{
7. The granularity of medical narratives and its effect on the speed and completeness of information retrieval
}

\author{
H.J. Tange ${ }^{a}$, H.C. Schouten ${ }^{b}$, A.D.M. Kesterc, A. Hasman ${ }^{a}$ \\ ${ }^{a}$ Department of Medical Informatics, Maastricht University \\ bepartment of Internal Medicine, Maastricht University Hospital \\ ' Department of Methodology \& Statistics, Maastricht University
}

\begin{abstract}
The incorporation of medical narratives in an electronic medical-record system is difficult. Most research has been conducted on the questions how to enter and store such data. How to retrieve narrative information from an electronic medical-record systen has not much been studied. Searching can be facilitated by dividing medical narratives into indexable paragraphs and organising these paragraphs according to different wiews. Well-. known combinations of views are source-oriented and problemmoriented flowsheets. In this chapter we study how medical narratives should be partitioned to support the physician optimally with retrieving information from medical narratives, when using an electronic medical-record system. In a simulative selting, we studied the speed of searching and completeness of retrieved information of thirty-six physicians from the internal department of an academic medical hospital. We compared three medical-record types that differ in the granularity of indexable paragraphs in which medical narratives are presented. The plysicians searched fastest in the intermediate structure, which was characterised by medical-history and physical-examination sections with paragraphs on the level of organ systems, and by progress notes divited into problem-sections. In the given setting, we did not encounter any effect of the granularity on the completeness of retrieved information. Furiher study, in real-life practice, is needed to validate our conclusions under other circumstances.
\end{abstract}

Submitted for publication 


\subsection{Introduction}

The primary function of the medical record is to supply information for direct medical practice. This function, originally meant to serve as an aide memoir for the individual physician, is more and more understood as providing a communication medium between different professionals sharing responsibility for the individual patient. Computerisation of the medical record is considered inevitable, but is impeded by several causes. The inability of existing electronic medical-record systems to incorporate physician-gathered medical narratives in a satisfactory way is seen as one of the major causes. ${ }^{\prime}$

For long, the question how to enter and store medical narratives was a matter of a choice between flexibility and control of medical expression. On one hand there was free-text data entry, which ensured maximal flexibility of expression, but without any control of its semantic consistency. On the other hand, maximal control could be achieved by using pre-formatted data-entry forms permitting only predefined answers to the questions on the form. This alternative, however, missed the flexibility to be useful outside a few restricted medical domains with rather constant information needs. Several classical systems implemented both approaches and captured medical narratives partly via predefined forms, partly as free text. ${ }^{2 \cdot 5}$ More recent approaches, however, strive for a combination of flexibility and control. Experiments are carried out with free-text data entry followed by natural-language interpretation. ${ }^{6-8}$ Another approach is known as predictive or dynamic data entry: the provision of pre-formatted forms or menus for controlled data input that automatically accommodate to the context, by anticipating the next input based on previously entered data. ${ }^{9.14}$ With both these approaches, promising results have been obtained. Both approaches, however, require the use of a highly expressive and covering controlled medical terminology. Only recently, appropriate terminologies have become available. ${ }^{15-17}$

Little attention has been paid, so far, to ways of facilitating the retrieval of information from electronic medical-record systems. It is assumed that information retrieval is much faster when using electronic medical-record systems instead of paperbased medical records, ${ }^{1819}$ although evaluation studies on this subject are scarce. Source-oriented and problem-oriented flowsheets, commonly used in existing electronic medical-record systems, are considered helpful searching structures to facilitate information retrieval. ${ }^{20}$ The inclusion of medical narratives in flowsheets, however, is less common. The few systems that include narratives in flowsheets usually present these data in coded format. ${ }^{21.22}$ Many other systems, however, present medical narratives as fluent prose (even if they have to be generated from coded data), ${ }^{2.4 .10 .14}$ which does not fit well in a flowsheet. We believe that accessing free-text medical narratives via flowsheets would be a step forward in the facilitation of information retrieval. In this chapter, we examine the effect of the paragraph size of free-text medical narratives on the ease of information retrieval. 


\subsection{Used terms and purpose of the study}

The process of information retrieval from the medical record can be divided into two steps. First, the user has to search through the medical record - guided by its internal (searching) structure - to select the item that might contain relevant information. Second, he has to read the content of this node to retrieve the information needed. By the searching structure of the medical record we mean its inner organisation in paragraphs designed to facilitate the retrieval of information. The searching structure functions as an index and is based on a combination of organising principles, each of which provides wiew (perspective) on the content of the medical record. In Chapter 4 we distinguished four views: the time view, source view, problem view, and process view. These views have in common that they are composed of elementary units of medical data. Numeric data (e.g., laboratory results, temperature measurements) are usually presented the way they were recorded, namely as single observations.

Medical narratives are clinicall data that are traditionally recorded in natural prose. In this study we restrict the term to medical history (in particular present illness and systems review), physical examination, and progress notes. In contrast with numeric data, medical narratives are usually neither recorded nor presented as separate observations. Instead, they may be diwided into indexable paragraphs. These paragraphs may differ in granularity. The granularity of medical narratives deternines the resolution of the searching structure, and is expected to influence the physician's capability of information retrieval.

In this chapter we report a study we carried out to determine the oplimal granularity for the presentation of medical narratives. We tried to answer the question how medical narratives should be partitioned to support the physician optimally when retrieving information for direct medical practice. Our hypothesis was that physicians search faster and obtain more complete information when using medical narratives with a high granularity (divided into small paragraphs).

\subsection{Method, materials, and test design}

We conducted an experiment in which we compared the physicians" capability of retrieving information from an electronic medical record, in terms of speed and completeness, using searching structures with three different granularities. The retrieval of information in medical practice may be influenced by many factors. To control these factors as much as possible, we chose for a simulation study in which a number of physicians had to solve some predefined problems with respect to three preselected, voluminous, inpatient cases. We conducted the study in the domain of internal medicine, because internal medicine is the "mother" of many other specialties and harbours a relatively large collection of voluminous patient cases.

\subsubsection{Medical-record types}

From descriptions in the literature of existing electronic medical-record systems, we concluded that medical history and physical examination are presented in three different granularities: (1) without partitioning, ${ }^{23-25}$ (2) with paragraphs on the level of 


\begin{tabular}{l|ll} 
& $a$ & $b$ \\
1 & $A$ & \\
2 & & $B$ \\
3 & & $C$ \\
\hline
\end{tabular}

medical-record types med hist / phys exam: progress notes:
$A=$ coarse, $B=$ intenthediafe, $Q=$ fine

$1=$ undivided 2 = organ systems, $3=$ separate observations

$a=$ undiwided, $b=$ problem sections

Figure $7-1$.

Medical-record types $(A, B, C)$ defined as combinations of granularity of medical histony and physical examination $(1,2,3)$ and of progress notes $(a, b)$.

organ systems, ${ }^{10,26}$ (3) partitioned into separate observations or even single findings. 21.25 .27 Progress notes are presented in two different granularities: (a) undivided ${ }^{10}$ and (b) partitioned into problem sections. ${ }^{\prime}$ Progress notes may also have a process-oriented structure (SOAP). ${ }^{28}$ However, we decided not to regard this SOAP. structure as a third level of gramularity of progress notes for two reasons. Firsi, SOAP is not necessarily related to problem-partitioning: in the literature, also undivided progress notes with a SOAP-structure have been described. ${ }^{10,29}$ Second, when using medical history and physical examination with granularities (2) and (3), SOAP will be fragmented among medical history, physical examination, and progress notes, leaving only the assessment part for the progress notes, thus obviating the need for a further subdivision. ${ }^{30}$

In Figure $7-1$ the different gramularities of medical history and physical examination (in rows) and of progress notes (in columns) are presented. For our study, we selected the three most encountered combinations of granularity, which resulted in three medical-record types.

Type A: Coarse medical-record type - Medical history, physical examination, and progress notes are all undivided (combination 1a). Observations during intake are included in the medical history and physical examination, while similar observations performed after intake are recorded in the progress notes. This structure is similar to the partitioning of the traditional, paper medical record.

Type B: Intermediate medical-record type - Medical history and physical examination are paragraphed according to organ systems, and progress notes are divided into problems (combination 2 b).

Type C: Fine medical-record type - Medical history and physical examination are partitioned into separate questions and observations, respectively, while progress notes are, again, divided into problems (combination 3 b).

As explained before, in both the intermediate and fine medical-record type, observations performed after intake were not located in the progress notes, but in the paragraphs of medical history and physical examination where also the corresponding observations during intake could be found. 
Table $7-1$ :

Source view-Differences in granularity of the three medical-record types used.

The items in italic are the elementary paragraphs that contain patient data. The number of en-dashes $(-)$ indicates the hierarchic level of the paragraphs.

\begin{tabular}{|c|c|c|}
\hline Coarse structure (A) & Intermediate structure (B) & Fine structure (C) \\
\hline $\begin{array}{l}\text { medical history } \\
\text { physical examination } \\
\text { progress notos }\end{array}$ & $\begin{array}{l}\text { medical histon } \\
\rightarrow \text { general wefl-being } \\
\rightarrow \text { choulatory system } \\
\rightarrow \text { respiratony system } \\
\rightarrow \text {... } \\
\text { physical examinalion } \\
\rightarrow \text { general impression } \\
\rightarrow \text { head and neck } \\
\rightarrow \text { heart } \\
\rightarrow \text { lungs } \\
\rightarrow \text {.... } \\
\text { progress notes } \\
\rightarrow \text { problem } 1 \\
\rightarrow \text { problem } 2 \\
\rightarrow \text {... }\end{array}$ & $\begin{array}{l}\text { medical history } \\
\rightarrow \ldots \\
\rightarrow \text { circulatory system } \\
\rightarrow \rightarrow \text { palpitations } \\
\rightarrow \rightarrow \text { chest pain } \\
\rightarrow \rightarrow \ldots \\
\text { physical examination } \\
\rightarrow \ldots \\
\rightarrow \text { Hungs } \\
\rightarrow \rightarrow \rightarrow \text { percussion } \\
\rightarrow \rightarrow \text { auscultation } \\
\rightarrow \ldots \\
\text { progress notes } \\
. \text { problem } 1 \\
\rightarrow \rightarrow \text { problem } 2 \\
\rightarrow \ldots\end{array}$ \\
\hline
\end{tabular}

Table $7-2$ :

Problem view-Selection of narrative paragraphs relevant for the problem "liver cirrhosis".

Differences in granularity of the three medical-record types used. The items in italic are the elementary paragraphs that contain patient data. The number of en-dashes (-) indicates the hierarchic level of the paragraphs.

\begin{tabular}{|c|c|c|c|}
\hline Coarse structure & Intermediate structure & Fine struclure & fine structure (continued) \\
\hline $\begin{array}{l}\text { medical history } \\
\text { physical examination } \\
\text { progress notes }\end{array}$ & $\begin{array}{l}\text { medical history } \\
\rightarrow \text { general well-being } \\
\rightarrow \text { digestive system } \\
\rightarrow \text { urogenital system } \\
\rightarrow \text { dermatologic } \\
\rightarrow \text { neurologic } \\
\text { physical examination } \\
\rightarrow \text { general mpression } \\
\rightarrow \text { abdomen } \\
\rightarrow \text { skin } \\
\text { progress notes } \\
\rightarrow \text { liver cirhosis }\end{array}$ & $\begin{array}{l}\text { medical history } \\
\rightarrow \text { general well-being } \\
\rightarrow \rightarrow \text { tired? } \\
\rightarrow \text { digestive system } \\
\rightarrow \rightarrow \text { colour stool? } \\
\rightarrow \text { urogenital system } \\
\rightarrow \rightarrow \text { colour urine? } \\
\rightarrow \text { dermatologic } \\
\rightarrow \rightarrow \text { fching? } \\
\rightarrow \text { neurologic } \\
\rightarrow \rightarrow \text { diziness? }\end{array}$ & $\begin{array}{l}\text { physical examination } \\
\rightarrow \text { general impression } \\
\rightarrow \rightarrow \text { in-looking? } \\
\rightarrow \rightarrow \text { jaundice? } \\
\rightarrow \text { abdomen } \\
\rightarrow \rightarrow \text { percussion Mver } \\
\rightarrow \rightarrow \text { percussion spleen } \\
\rightarrow \rightarrow \text { percussion elsewhere } \\
\rightarrow \rightarrow \text { palpation liver } \\
\rightarrow \rightarrow \text { palpation spleen } \\
\rightarrow \text { skin } \\
\rightarrow \rightarrow \text { scratching manks } \\
\text { progress notes } \\
\rightarrow \text { liver cirmosis }\end{array}$ \\
\hline
\end{tabular}

All three medical-record types provided a searching structure based on the source view, problem view, and time view (as defined in Chapter 4). The source view is a hierarchical list of paragraphs organised according to patient-independent criteria. Main criterion is the source of data. In the record types (B) and (C) we organised the paragraphs within medical history and physical examination according to the usual procedure of data gathering (see Table 7-1). The problem view is a list of paragraphs that can be relevant to the given problem. For each problem encountered in the cases 


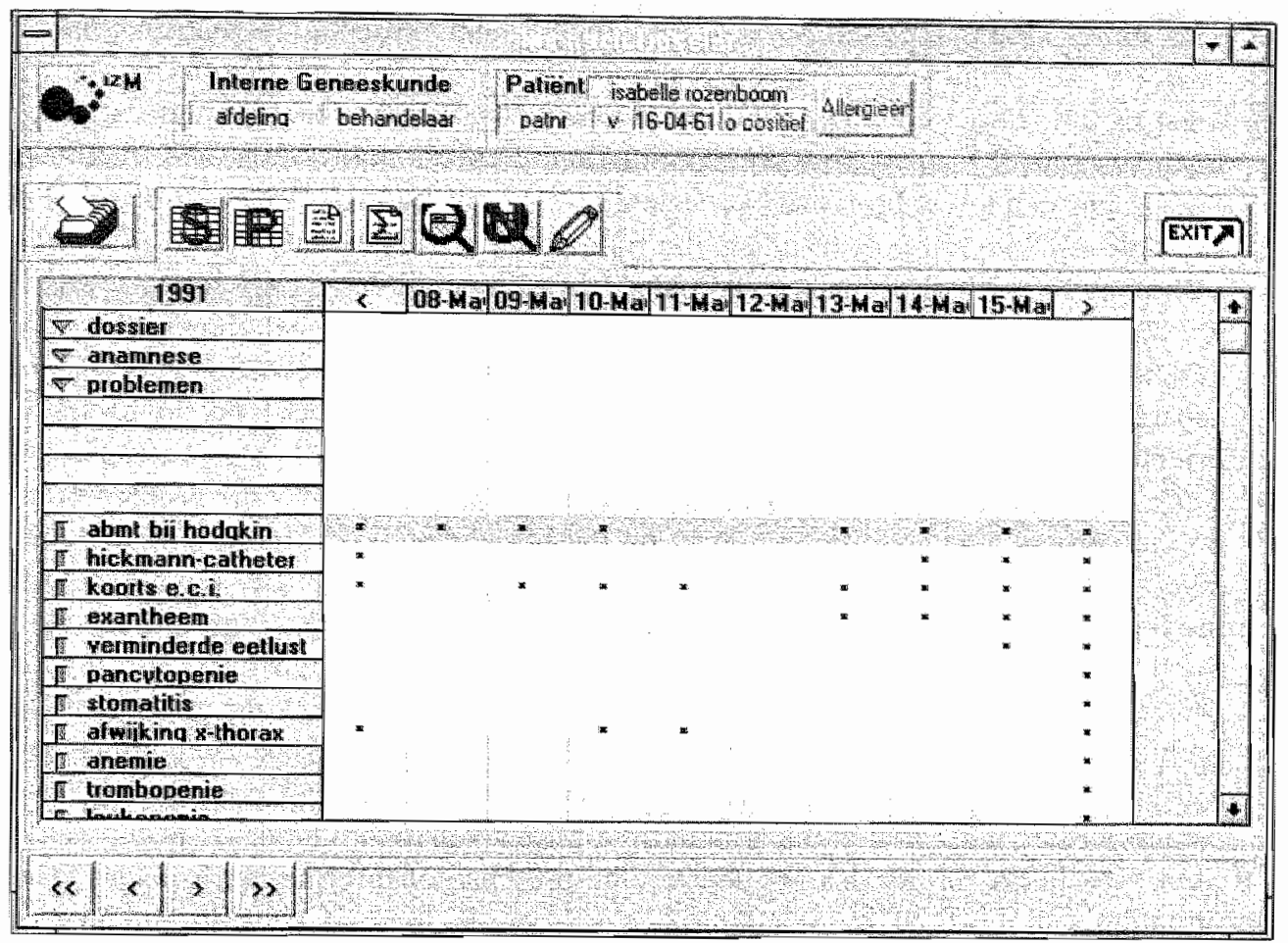

Figure 7-2:

Problem-oriented flowsheet-Top level with problem list and corresponding progress notes. In this example, the problem "autologous bone-marrow transplantation" ["abmt bij hodgkin"] is selected over the entire time range. From this point on, the user can access the selected progress notes for reading or jump to the lower level of the flowsheet, which will show a list of the problem-relevant paragraphs.

used, we reconstructed a problem view from problem-related recordings written down in the original paper medical record. For example, the problem view of the problem liver cirrhosis is demonstrated in Table 72 . The problem views were subsequently validated by an extemal referent (an internist). For a full description of the source view and problem view see the appendix. The time view we used had a fixed seale of days.

\subsubsection{The electronic medical-record system}

All three medical-record types were presented via one electronic medical-record system. Hence, they could be accessed through the same, standard, graphical userinterface. The system was developed in Visual Basic $3.0^{\mathrm{TM}}$ in the MS-Windows $3.11^{\mathrm{Tm}}$ environment. The medical-record types were specified in a Btrieve $5.0^{\mathrm{rm}}$-database in which also the patient data were stored. We used Btrieve because it is very fast and flexible. Medical narratives were stored as free text within the given paragraphs. Advanced text-processing functions to facilitate searching were not available. For a detailed description of this system see Chapter 6 . Here, we only describe the screens relevant for retrieving information from the medical record in general, and from medical narratives in particular. 
- The sourcemoriented flowshet is a two-dimensional presentation matrix with the source view on the vertical axis and the time view on the horizontal axis. The top level of the source view consists of the following sections: background data, past history, medical history, physical examination, progress notes, tests, and treatment. Paragraphs at a lower level can be reached by a simple mouse click on a section. For example, clicking on tests results in the following list: clinical laboratory, radiology, pathology, etc. Medical history and physical examination have only lower levels in the record types (B) and (C). The second level of the flowsheet shows the organ systems and body regions, and the third level shows, only in record type (C), the separatc observations. Clicking on the progress notes gives access to the problemoriented flowsheet.

- The problem-oriented flowsheet is a two-dimensional matrix with the problem view on the vertical axis and the time view on the horizontal axis (Figure 7-2). The top level of the problem view presents the patient's problem list. In the columns next to the list the progress notes are presented. In record type (A) the progress notes next to all problems of the list are equal (undivided). In the record types (B) and (C) the progress notes next to each problem are problem-related. Clicking on one of the problems in the list gives access to the second level of the flowsheet, which presents a list of paragraphs that is considered relevant for the problem concerned. These paragraphs are presented in the granularity of the given record type.

In both flowsheets, an asterisk $(*)$ denotes the presence of information in a cell. This information can be read by selecting one cell or an area of cells in the matrix. Subsequently, the content of the selected cells are collated and presented in browsable text window (the reading window), including their paragraph headings. Per default, medical data in the reading window are presented in clescending time order (which can be inverted by the user).

\subsubsection{Patient cases}

We used three patient cases from the domain of internal medicine, selected by one of the authors (HCS) at a moment that he was not yet acquainted with the electronic medicalrecord system nor" with the medical-record types under study. He used the following criteria for selection:

- The cases should be large and complex. The hospitalisation period should last more than three weeks, and at least ten different active medical problems should be recognised, of which at least three should be manageable with only basic knowledge of internal medicine.

- Hospitalisation took place more than one year ago, to minimise the risk of recognition (the patients had been hospitalised at the same department from whilch also the test persons were recruited).

The selected cases, originally documented in paper medical records, were entered into the computer by the investigator (HJT). The medical narratives were entered in the coarse record type (A) as an exact copy of the paper medical record, and divided into paragraphs in the intermediate (B) and fine record type (C). Results from auxiliary services of the first case were entered manually. For the other cases, a procedure was developed to download them automatically from the hospital information system. 
Table 7-3:

Problems addressed in the questions.

\begin{tabular}{lccc}
\hline Addressed problem & case $(x)$ & case $(y)$ & case $(z)$ \\
\hline fever, cause unknown & $*$ & $*$ & $*$ \\
protocol compliance & $*$ & & \\
stomatitis & $*$ & $*$ & $*$ \\
liver cirhosis & & $*$ & $*$ \\
renal failure & & $*$ \\
fluid retention & & $*$ \\
thrombopenia & & & \\
leg uicer & & & \\
\hline
\end{tabular}

Patient $(\mathrm{x})$ was a 30 -year old woman known for many years with Hodgkin's disease. She was hospitalised to undergo an autologous bone-marrow transplantation. Patient (y) was a 47-year old man known with liver cirrhosis caused by alcolhol abuse who was hospitalised suspected of venous thrombosis (which could not be verified). During hospitalisation, he developed an end-stage renal failure. Patient $(z)$ was a 53year old woman who was hospitalised suspected of leukaemia. During hospitalisation, the diagnosis acute myeloid leukaemia was established and the woman received her first chemotherapy.

\subsubsection{Situations for consulting the medical record and questions for searching}

During the test, the test persons were confronted with a number of situations concerning an "unknown" patient which may occur when (re)considering medical policy (when on call, on round, or on consultation) or delivered care (when composing a discharge summary, or for medical audit purposes). For each situation, one or more specific questions were formulated that could have been raised in reality. Also these situations and questions were formulated by one of the authors (HCS) at a moment that he was not yet acquainted with the electronic medical-record system nor with the medical-record types under study. He based his descriptions on the following considerations.

- A illustrative set of retrieval situations should be addressed. To get an impression of which reasons for consulting the medical record are common, an inventory was made of the reasons for call during two successive weeks. From a total of $24 \mathrm{calls}$, $18(75 \%)$ had one of the following reasons: high fever (5), abnormal test results (5), dyspnea (4), or cardiac complaints (4).

- The questions should mainly ask for narrative information.

- The questions should not appeal for medical knowledge that was not expected to be present in all test persons. 
Specification and characterisation of the questions for searching.

\begin{tabular}{|c|c|c|}
\hline $\operatorname{cose}(x)$ & woman 30; Hodgkin's disease; autologous bone marrow transplantation & categary \\
\hline day 10 : & $\begin{array}{l}\text { You are on duty and you are called by a nurse of the haemato-oncology } \\
\text { department Mrs. (x) has got fever. }\end{array}$ & \\
\hline 1. & What was the indication for hospitalisation? & 1 \\
\hline 2. & Which diagnoses and treatrments were given in the past? & $i$ \\
\hline 3. & What was the initial treatment plan after intake? & II \\
\hline 4. & History and physical examination are negative. Make up a differential & 11 \\
\hline dav 29: & $\begin{array}{l}\text { diagnosis for the cause of the fever (at least } 3 \text { options). } \\
\text { Discharge }\end{array}$ & \\
\hline \multirow[t]{2}{*}{$>$ distischarge. } & Mrs (x) has been discharged. You saw her only the fasit wo days and you & \\
\hline & must dictate the discharge letter. & \\
\hline 5. & How many cells were in the bone marrow transplant? & II \\
\hline 6. & $\begin{array}{l}\text { Which was, in retrospect, the cause of the stomatitis that started on the } 15 \text { th } \\
\text { day, and how was it treated. }\end{array}$ & 11 \\
\hline 7. & $\begin{array}{l}\text { Which was in retrospect, the cause of the fever that occurred on the foth } \\
\text { day? }\end{array}$ & II \\
\hline Case $(y)$ & man 47: liver cirnosis with alconol abuse; renal failure & category \\
\hline day $2:$ & $\begin{array}{l}\text { Mr. (y) was hospitalised yesterday via the emergency ward. Today you see } \\
\text { him for the first time, being the physician of the inpatient ward. }\end{array}$ & \\
\hline 8. & Were there signs of ascites found in the emergency ward? & III \\
\hline 9. & Were there signs of ascites found in the past? & 1 \\
\hline 10. & $\begin{array}{l}\text { Which were, yesterday, the considerations for requesting a bone marrow } \\
\text { puncture? }\end{array}$ & $\|$ \\
\hline 11. & $\begin{array}{l}\text { Mr. (y) has symptoms at hands and feet. How were they designated and what } \\
\text { was done to support this diagnosis? }\end{array}$ & 11 \\
\hline day 21: & You are on duty and you are called because Mr. (y) has got fever. & \\
\hline 12 & $\begin{array}{l}\text { What do you find during physical examination and what is the most likely } \\
\text { cause of the fever? }\end{array}$ & $\| 11$ \\
\hline day 56 : & $\begin{array}{l}\text { It is considered to transier Mr (y) to a nursing home. You are asked for a } \\
\text { second opinian. }\end{array}$ & \\
\hline 13. & $\begin{array}{l}\text { You read that Mr }(y) \text { has a renal failure. Which tests were performed to } \\
\text { determine its aetiology, and which diagnosis has been established? }\end{array}$ & $\|$ \\
\hline 14. & $\begin{array}{l}\text { You read about a positive blood culture with staphylococcus aureus. Yet, the } \\
\text { treatment with flucloxicilline was interrupted and replaced by other antibiotics. } \\
\text { Why? }\end{array}$ & II \\
\hline Case (z) & woman 53; acute myoloid leukaemia; diagnosis and first chemotherapy & category \\
\hline day 12 : & $\begin{array}{l}\text { You are on weekend duty and are making round's. Mrs. (z) lost } 13 \text { kilo's within } \\
1 \text { week. }\end{array}$ & \\
\hline 15 & $\begin{array}{l}\text { Did the diuretic treatment exceed its goal or not? Find also treatment } \\
\text { considerations to support your answer. }\end{array}$ & $\| 11$ \\
\hline day $23:$ & You are on duty and read a remark on day 22 "no haematuria any more". & \\
\hline 16. & $\begin{array}{l}\text { Since when were there signs of haematuria, and what was considered as its } \\
\text { cause? }\end{array}$ & 111 \\
\hline \multirow{2}{*}{$\begin{array}{l}\text { day } 27 \text { : } \\
\text { s discharge: }\end{array}$} & Discharge & \\
\hline & $\begin{array}{l}\text { Mrs (z) has been discharged. You saw her only the last two days and must } \\
\text { dictate the discharge letter. }\end{array}$ & \\
\hline \multirow{2}{*}{$\begin{aligned} 17 \\
\text { discharge: }\end{aligned}$} & What was considered the cause of the leg ulcers and how were they treated? & II \\
\hline & Mrs (z) has been discharged. You are auditing protocol adherence. & \\
\hline 18 & $\begin{array}{l}\text { Did Mrs }(z) \text { receive GM-CSF, as a part of the protocol? Why was this } \\
\text { treatment mot accomplished conform the protocol? }\end{array}$ & 11 \\
\hline 19. & $\begin{array}{l}\text { You are looking for complications of the treatment. Were there signs of } \\
\text { congestive heart failure, during the treatment or before? }\end{array}$ & III \\
\hline
\end{tabular}


Table 7-5:

Example of a question with an answer consisting of three essential data elements. Gold standard existing of three elements. each element retrieved amounts to $33 \%$.

Gold standard (retrieved from progress notes)

You read that Mr (y) has a renal failure. Which tests were performed to determine its aetiology, and which diagnosis has been established?

\section{Dec sediment}

17 Dec biopsy (performed on $12^{\text {in }}$ )

17 Dec mesanglal proliferation / end-stage glomerulo-nephritis (performed on $12^{\text {itu }}$ )

- The questions should be of various kinds. In a study observing seven specialists from different disciplines, Nygren and Henriksson determined four strategies of reading the medical record: (1) to get an overview of an unknown patient case, (2) to refresh one's memory about a known patient case, (2) to search for specific data, and (4) to solve medical problems (i.e., to generate and test hypotheses). ${ }^{3}$ In a covering set of questions all these purposes should be addressed. In our study, however, the second purpose is not addressed because the patient cases were unknown to the test persons.

Table 7-3 illustrates which problems were addiressed in the reasons for consulting the medical record. Most situations described were replicas of what really had happened. Table 7-4 shows the questions in detail. Seven questions were formulated about case $(x)$, seven about case $(y)$, and five about case $(z)$. The questions were tested for possible ambiguities on six randomly chosen specialists (see also 7.3.10).

\subsubsection{Test population}

We recruited our test persons from the Department of Internal Medicine of Maastricht University Hospital. Candidates were physicians with sufficient experience in internal medicine, i.e., all specialists in this discipline and those residents that were qualified as such by one of their supervisors. We restricted the study to an academic sefting, because such a setting harbours a broad spectrum of medical experience and pays relatively much attention to medical documentation.

\subsubsection{Outcome measures and outcome model}

We were interested in the effect of the medical-record type on the speed of information retrieval and the completeness of retrieved information. To determine the speed we observed the time passed between the first and last computer action that a test person performed while answering a question. To determine the completeness of a given answer we calculated the proportion of essential data elements that were actually

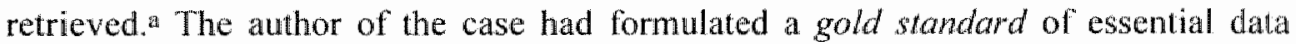
elements for each question. He did this on the basis of his knowledge of the case and a review of the answers given by the first ten test persons. When reviewing these

\footnotetext{
a This meastre is also known as recall. Its counterpart, preciston, was not studied since our datagathering procedure (see 7.3 .10 ) was not precise in itself: the test persons had to mark relevant text fragments with a mouse device, but it was difficult for many of them to take the exact begin and end of a drag action.
} 
answers he was blinded with respect to test person and medical-record type. An answer could consist of one or more data elements from the medical record. (Table 7-5). All information essential for the question was considered equally important. For example, one element out of four amounted to a completeness of $25 \%$.

For each test person and each question we recorded the time spent for searching and the proportion of essential information retrieved. Thus, for each test person nineteen values of speed and of completeness were obtained. As outcome measures for the main analysis of this study we took, for each test person, the averages of the observed values per medical record-types. In this way, for each test person three outcome measures for speed and for completeness were obtained.

Apart from the effect of interest (medical-record type) we identified some other effects that could also influence the outcome measures.

1. Individual variations between test persons, such as test attitude and computer skills, could have a direct effect on their performance of the test. Moreover, it has been demonstrated that the strategy of medical thinking depends on medical expertise: medical students and less experienced physicians use a more procedural work-up, while experienced physicians rely more on rules of thumb. ${ }^{32}$ Different thinking strategies may lead to different searching strategies which, in turn, may be supported best by different medical-record types. Thus, medical expertise could influence the outcome measure indirectly, by modifying the effect of the medicalrecord type. As a criterion for medical expertise we distinguished between specialists and residents.

2. The nineteen questions to be answered would inevitably differ in complexity, which could have a direct effect on the outcome measures. These differences between questions could be carried over to the three patient cases.

3. We faced the problem that all test persons would use the system only once, so we had to account for a learning effect during the test, in favour of the last medical record-type the test persons were confronted with. As a criterion for this direct effect on the outcome measures we took the series of the record type in the test.

Based on the discussion above, we designed the following outcome model (Table 7-6). The mean outcome is the average of all outcomes. Main effects are factors that influence the outcome of the test directly (among which the medical-record type, our effect of interest). Interactions are effect modifiers, i.e., factors that influence the effect of the record type on the outcome. The residual error is the accidental variation of the outcome.

Table 7-6:

Outcome model of the main analysis. For each test person three outcomes are obtained. Number of outcomes per outcome measure: $36 * 3=108$.

\begin{tabular}{cl}
\hline Outcome & $=$ mean outcome * main effects + interactions + residual error \\
with & \\
main effects & $=$ test person + record type + patient case + series \\
interactions & $=$ medical expertise $\rightarrow$ record type + patient case $\rightarrow$ record type + series $\rightarrow$ record type
\end{tabular}




\subsubsection{Crass-over design, sample size, and sampling procedure}

To balance the main effects in the outcome model we used a crosingwer destgry for three treatments. This is a usual design for clinical trials in which the affect of wh alternative treatments is compared with a placebo or a gold standard. ${ }^{33.34}$ To a certain extent, our study design is comparable with a that of a clinical trial. Only the subjects we used were physicians instead of patients and the alternatives we compared were record types instead of therapies.

A completely balanced design, by which each test person is given each combination of medical record type and patient case, was not possible because in that case the test persons would be confronted with the same patient (and also the same questions) three times. The specific allocation pattern we used is known as balanced incomplete block design (Table 7-7). A balanced set of outcomes would be reached. with thirty-six test persons. In each series, each of the three medical-record types is presented twelve times, as is each of the three patient cases. Taking into account the numbers of specialists and residents employed in the department under study ( 35 and 18 , respectively), we stratified the set of test persons into 24 specialists (four blocks) and 12 residents (two blocks). For each stratum we used a randomly generated list of candidates for inviting them to participate. At the beginning of the test, each participant was randomly assigned to one of the sequences in the block design.

Table 7-7

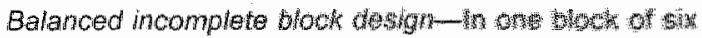

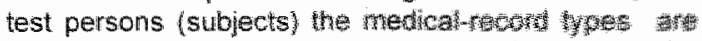

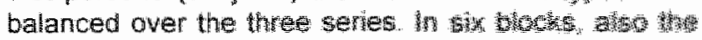
patient cases are balanced.

Number of test persons needed: $6: 6=3$

\begin{tabular}{|c|c|c|c|c|}
\hline Subject & Expertis & Serres & Serista 2 & 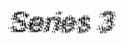 \\
\hline 1 & $s$ & $A x$ & by & $t_{02}$ \\
\hline 2 & $\mathrm{~s}$ & $A x$ & $a y$ & $\mathrm{Bz}$ \\
\hline 3 & s & $B x$ & $A y$ & SE \\
\hline 4 & $s$ & $8 x$ & Cy & $A z$ \\
\hline 5 & s & $C x$ & Ay & $B z$ \\
\hline 6 & s & $\mathrm{Cx}$ & $8 y$ & 喵 \\
\hline 7 & a & $A x$ & Bz & Sy \\
\hline 8 & a & $A x$ & $\mathrm{Cr}_{2}$ & $8 y$ \\
\hline 9 & a & $B x$ & $\mathrm{Az}$ & $\mathrm{B}$ \\
\hline 10 & $a$ & $8 x$ & $\mathrm{Cz}$ & $A x$ \\
\hline 11 & a & $C x$ & $A z$ & $8 y$ \\
\hline 12 & a & $C x$ & $B z$ & Hy \\
\hline 13 & s & Ay & $9 x$ & $\mathrm{Cx}_{2}$ \\
\hline 14 & s & Ay & $C_{x}$ & at \\
\hline 15 & s & $8 y$ & $\mathrm{Ax}$ & 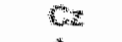 \\
\hline 16 & s & By & $\mathrm{Cx}$ & 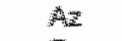 \\
\hline 17 & $s$ & $\mathrm{Cy}$ & $A x$ & $E^{2}$ \\
\hline 18 & s & Cy & $B$ & ${ }_{A} \mathrm{E}$ \\
\hline 19 & $a$ & Ay & $\mathrm{Bz}$ & $\mathrm{px}$ \\
\hline 20 & a & Ay & $C_{x}$ & $8 x$ \\
\hline 21 & a & By & $A=$ & $C_{*}$ \\
\hline 22 & a & By & $\mathrm{Cz}$ & $A x$ \\
\hline 23 & a & Cy & $A z$ & $\mathrm{Bx}$ \\
\hline 24 & a & Cy & $\mathrm{Bz}$ & $A x$ \\
\hline 25 & $s$ & $A z$ & $B x$ & Cy \\
\hline 26 & 5 & $\mathrm{~A} Z$ & $\mathrm{Cx}$ & By \\
\hline 27 & $s$ & $\mathrm{Bz}$ & $A x$ & $\mathrm{Cy}$ \\
\hline 28 & s & $\mathrm{Bz}$ & $C x$ & Ay \\
\hline 29 & $\mathrm{~s}$ & $\mathrm{Cz}$ & $A x$ & By \\
\hline 30 & s & $\mathrm{Cz}$ & $B x$ & Ay \\
\hline 31 & s & $A z$ & By & $C x$ \\
\hline 32 & s & $\mathrm{Az}$ & $\mathrm{cy}$ & $B x$ \\
\hline 33 & s & $\mathrm{Bz}$ & $\mathrm{Ay}$ & $C x$ \\
\hline 34 & $s$ & $\mathrm{Bz}$ & $\mathrm{Cy}_{\mathrm{y}}$ & $A x$ \\
\hline 35 & $\mathrm{~s}$ & $\mathrm{Cz}$ & Ay & $\mathrm{Bx}$ \\
\hline 36 & $s$ & $\mathrm{Cz}$ & By & $A x$ \\
\hline
\end{tabular}

Series = ordier in which a record-type / patient-cease combitnation is offered $A_{x} x=$ record type $(A)$, patient $(x)$, etc. 

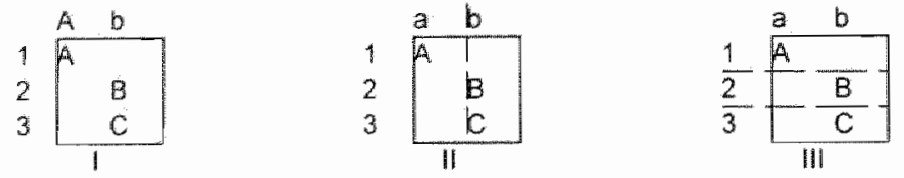

Figure $7-3$

Classification of questions into three categories (I, II, III) according to the type of information they ask for. The last column of Table $7-4$ shows which questions belong to which category.

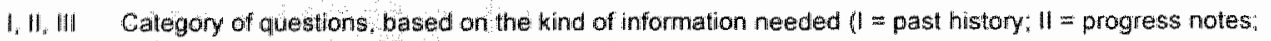
III = medical history ( physical examination).

A, B, C Medical-record lypes $(A=$ coarse; $B=$ intermediate; $C=$ fine) as defined in Paragraph 7.3.1.

$1,2,3$ Medical history and physical examination ( $1=$ undivided $2=0$ organ system; $3=$ separate obserwations).

$a$ b Progress notes $(a=$ undivided $b=$ problem-oriented).

\subsubsection{Statistical analysis}

The outcomes obtained were analysed in SPSS $5.1^{\mathrm{TM}}$ conducting an more-way analysis of variance (ANOVA). With this technique it is possible to examine, in one single design, the influence of several sources of variance on a dependent variable, both directly (as main factors) and in interaction with one another. ANOVA is a parametric technique assuming normally distributed residual errors.

Significant sources of variance $(\mathrm{p}<0.05)$ were further analysed to determine which individual values were responsible for this variance. If, for example, the medicalrecord type would have a significant effect on search speed, the differences between the individual record types (A-B, A-C, B-C) would be tested. These differences were tested with a t-test, which is part of ANOVA under the option pair-wise simple contrast analysis. ${ }^{35}$

\subsubsection{Sub-analysis}

In Paragraph 7.3.1 we described three possible granularities in medical history and physical examination, and two possible granularities in progress notes. We only studied the three most encountered of the six possible combinations of granularities. Hence, a record-type effect (when found) could be caused by differences in the granularity of medical history and physical examination, by differences in the granularity of progress notes, or by both. To distinguish between these aspects we conducted a sub-analysis.

The last column of Table 7-4 demonstrates that the nineteen questions of the test could be divided into three categories according to the type of in formation they asked for. Three questions asked for information about the past history, which could be found in a separate section of the medical record (category I). Eleven questions asked, (among others), for information about clinical jud gement, which was presented in the progress notes (category II). Five questions asked for information from observations that could be found in the sections medical history and physical examination (category (II).

Figure $7-3$ demonstrates how the different categories distinguish between the granularity of medical history and physical examination on one hand and the granularity of progress notes on the other. The past-history section (category I) was 
Table T-8:

Outcome model of the sub-analysis. For each test person nineteen outcomes are obtained. Number of autcomes per outcome measure: $36 * 19=324$.

\begin{tabular}{cl}
\hline Outcome & $=$ mean outcome * main effects + interactions * individual error \\
with & $=$ test person + record type + question + series effect \\
main effects & $=$ expertise $\rightarrow$ record type + categon $\rightarrow$ record type * series effect $\rightarrow$ record type
\end{tabular}

equal in all three medical-record types. The progress-notes section (category II) had the same structure in the intermediate $(B)$ and fine $(C)$ record type, but a different one in (A). The medical-history and physical-examination sections (category III) were different in all three medical-record types. To investigate the effect of partitioning progress notes we used the questions of category II; to investigate the effect of partitioning medical history and physical examination we used the questions of category III. It should be noted that in record type (A) medical-history and physicalexamination entries after intake were recorded in the progress notes instead of in their original sections (as is usual in the traditional paper-based medical record, see 7.3.1).

Effect of interest for this sub-analysis was the influence of the category of questions on the record-type effect, which is an indirect effect on the outcome of the test. We were not interested in its direct effect. We could not use the same outcome measures as we did in the main analysis, because there were no questions of category (I) for patient ( $z$ ) and no questions of category (III) for patient ( $\mathrm{x}$ ). Hence, we took as outcome measures the original observed values of each test person for each separate question (as described in 7.3.6). As a consequence, we used the question instead of the patient case as main effect. The outcome model for this sub-analysis is presented in Table 7-8. The approach of a balanced incomplete block design was maintained.

\subsubsection{Test configuration and data sampling procedure}

For the purpose of this study, we extended the electronic medical-record system (as described in 7.3.2) with some specific functions to support the test. We added an allocation procedure for test persons, a mouse-skill test, an automated protocol guiding the test persons through the sequence of questions and patient cases, and a procedure with which all computer actions of the test persons could be recorded for further analysis. This extended version of the electronic medical-record system was installed on a $\mathrm{PC}$-workstation in a separate room. To avoid disturbance, the test persons had to switch off their semaphone. The testing was supervised by the investigator (HJT).

At the beginning of the session, the test person had to enter some information abolst their medical and computer experience and performed a short mouse-skill exercise. The control of the program was explained using two questions concerning a dummy case, and subsequently the test persons could exercise with two more questions concerning this same case. During the real test, the test persons were guided by the program from one question to the next and from one patient to the other. Clinical information recorded after the period covered by the question was not presented. 
Talbe $7-9$ :

Duration of intormation retrieval, averaged over the test persons, as a function of record type, patient case, and series effect (geometric average, in seconds). Each average represents 36 test persons.

\begin{tabular}{ccc|ccc|ccc}
\hline Recordlype average SE mean & Palient case average SE mean & Series average SE mean \\
A & 152 & 9.0 & $x$ & 122 & 5.3 & 1 & 149 & 7.8 \\
B & 124 & 8.0 & $y$ & 117 & 4.5 & 2 & 134 & 8.2 \\
C & 131 & 5.8 & $z$ & 174 & 8.8 & 3 & 123 & 7.5 \\
\hline
\end{tabular}

SE mean = standard error of the mean

Each question was introduced by description of the reason for consultation. Measurements started when the test person switched to the medical record to search the information needed. From that time on, all user-driven actions were logged and time-stamped. If the test person found information that he used as evidence, he had to mark the given text with the mouse device. All computer actions, marked information, and possible additional comments were recorded in an MS-Access 1.1 TM research database, for further analysis. We used MS-Access instead of Btrieve, because its compatibility, via ODBC (open database connection), with SPSS $5.1^{\mathrm{TM}}$.

The test design and configuration were tested on six randomly selected specialists regarding possible ambiguities in the formulation of questions and possible incomplete recording of measurements. As a result, some adaptations were made. The results obtained with these six specialists were excluded from further analysis.

\subsection{Results}

The test was performed by 24 specialists and 12 residents. In total $80 \%$ of the approached specialists and $73 \%$ of the approached residents accepted to participate in the study. Non-co-operation was, in all cases, due to lack of time. The average duration of the entire test, including explanation and preparatory exercise, was 50 (38-68) minutes, of which $44(32-63)$ minutes were spent on the real test. On average, residents performed the test in 42 minutes, specialists in 45 minutes. All questions for searching were answered satisfactorily, according to the test persons.

\subsubsection{Speed of information retrieval}

Outcome measure for the speed of information retrieval was the average time for each recond type a test person needed to answer a question. In Table 7-9 this outcome measure is, in addition, averaged over the thirty-six test persons. The table shows that information retrieval was slowest when using the coarse medical-record type (A) and fastest when using the intermediate record type (B). Compared with the coarse typr, the intermediate type was, on average, 28 seconds (18\%) faster and the fine type 21 seconds $(16 \%)$. Remarkable is the very slow retrieval of information about patient $(z)$, the woman with leukaemia. The order of presentation suggests a learning effect.

To test the significance of each possible source of variance, we performed an analysis of variance (ANOVA) using the outcome model described in 7.3.6. Because the residual variance (individual error) showed a log-normal distribution, we carried 
Tabie $7-10$

ANOVA- test of significance for sources of wariance (main effects) and effect modifiers (interactions) with respect to speed of information retrieval.

\begin{tabular}{lrrrrr}
\hline Sources of variance & SS & DF & MS & $F$ & $P$ \\
main effects & & & & & \\
- test person & 0.42 & 35 & 0.01 & 1.81 & 0.023 \\
- series & 0.12 & 2 & 0.06 & 9.33 & 0.000 \\
- patient case & 0.66 & 2 & 0.33 & 49.73 & 0.000 \\
intecoractions type & 0.12 & 2 & 0.06 & 8.73 & 0.000 \\
- patient case * record type & 0.03 & 4 & 0.01 & 1.29 & 0.318 \\
- series * record type & 0.03 & 4 & 0.01 & 1.04 & 0.394 \\
individual error & 0.03 & 2 & 0.01 & 2.16 & 0.125 \\
\hline
\end{tabular}

$S S=$ sums of squares; $D F=$ degrees of freedom; $M S=$ mean squares $F=F$-ratio; $p=$ significance of $F$

out all ANOVA-statistics on a log-transformed scale. For the same reason we used, in Table 7-9, the geometric average of the outcome measures (which is the anti-log of the average of the logarithms).

The results of the analysis of variance is summarised in Table 7-10. This table shows that the factor with the largest effect on the speed of information retrieval was the patient case $(\mathrm{F}=47.30, \mathrm{p}=0.000)$. Also the series of presentation and the medicalrecord type were highly significant sources of variance (both $p=0.000$ ). The variance explained by the test persons was also significant, but relatively small $(\mathrm{p}=0.023)$. Table $7-10$ does not show evidence of any indireet effect (via the medical-recond type) on the speed of information retrieval.

Subsequently, we analysed the pair-wise differences between the individual values of the significant sources of variance (record type, patient case, and series effect) by means of a t-test. All significant effects had three possible values (two degrees of freedom), so there were always three possible pair-wise comparisons. Thus, according to Bonferroni"s correction for multiple comparisons, the required level of significance for all these pair-wise t-tests should be upgraded to $0.05 / 3=0.017 .^{36}$

From Table 7-11 it can be concluded, that searching with the coarse medical-record type (A) was significantly slower than with both other types (both $\mathrm{p}=0.000$ ). A significant difference between the intermediate $(B)$ and fine record type (C) could not be demonstrated. As was expected, retrieving information about patient ( $z$ ) took significantly more time than in the other two cases. A significant series effect could be

Table 7-11:

ANOVA: comparison of speed between separate record types, patient cases, and series (pair-wise t-test).

\begin{tabular}{cl|cc|cc}
\hline Record type & $p$-value & Patient case & $p$-value & Series effect & $p$-value \\
\hline A-B & $0.000 * *$ & $x-y$ & 0.326 & $1-2$ & 0.020 \\
A-C & $0.016 *$ & $x-z$ & $0.000 * *$ & $1-3$ & $0.000 * *$ \\
B-C & 0.102 & $y-z$ & $0.000 * *$ & $2-3$ & 0.060 \\
\hline
\end{tabular}

$*=$ significant< $<0.017$

$* *$ significant $<0.0017$ 
Table 7-12:

Completeniess of retrieved information, aweraged over the test persons, as a function of record type, patient case, and series effect ( in percentages of a gold standard). Each average represents 36 test persons.

\begin{tabular}{ccc|ccc|ccc}
\hline Record lype average SE mean & Patient case average SE mean & Series average & SEmean \\
A & 81 & 2.0 & $x$ & 87 & 1.5 & 1 & 81 & 2.0 \\
B & 81 & 2.0 & $y$ & 85 & 1.3 & 2 & 78 & 2.0 \\
C & 79 & 2.0 & $z$ & 70 & 1.8 & 3 & 82 & 2.0 \\
\hline
\end{tabular}

SE mean = standard error of the mean

demonstrated between the first and last series presented.

\subsubsection{Completeness of retrieved information}

The completeness of retrieved information or recall was defined as the proportion of relevant information that was found (see 7.3.6). Table 7-12 presents the average completeness of retrieved information (in percentages) as a function of the medicalrecord type and other possible sources of variance. The table shows averages around $80 \%$. The only remarkable entry is the relatively low proportion of information found about patient ( 2 ).

Table 7-13 summarises the results of the analysis of variance. This table does not demonstrate a significant effect of the medical-record type on the completeness of retrieved information, neither directly $(p=0.828)$ nor in interaction with another effect. The patient case was by far the largest source of variance ( $F=42.79, p=0.000)$. Of the other possible sources of variance, also the series effect was significant $(p=0.000)$, but the dip in the middle series (see Table 7-12) does not suggest a learning effect. By testing the differences among the individuall patients (t-test, $\mathrm{p}<0.017$ ) we found that the information retrieved about patient $(z)$ was significantly less complete than the information retrieved about $(\mathrm{x})$ and $(\mathrm{y})$.

Table 7-13:

ANOVA- test of significance for sources of variance (main effects) and effect modifiers (interactions) with respect to completeness of retrieved information.

\begin{tabular}{lrrrrr}
\hline Sources of variance & SS & DF & MS & $F$ & $\rho$ \\
\hline main effects & & & & & \\
- test person & 0.38 & 35 & 0.01 & 1.52 & 0.079 \\
- series effect & 0.03 & 2 & 0.01 & 1.94 & 0.000 \\
- patient case & 0.61 & 2 & 0.31 & 42.79 & 0.000 \\
intecord type & 0.00 & 2 & 0.00 & 0.19 & 0.828 \\
- pationt case * record type & 0.01 & 4 & 0.00 & 0.36 & 0.835 \\
- series effect * record type & 0.06 & 4 & 0.01 & 2.00 & 0.107 \\
- expertise * record type & 0.01 & 2 & 0.00 & 0.59 & 0.574 \\
individual error & 0.40 & 56 & 0.01 & & \\
\hline
\end{tabular}

$S S=$ sums of squares; $D F=$ degrees of freedom; $M S=$ mean $s q u a r e s ; F=F$-ratio; $P=$ significance of $F$ 
Table 7-14:

Categories of questions.

- Upper part: Description of categories of questions.

- Middle part: Duration of information retrieval, averaged over the test persons, as a function of medical record type, for each category of questions (geometric average, in seconds).

- Lower part ANOVA comparison of speed between separate record types (pair-wise t-test) for each category of questions.

\begin{tabular}{|c|c|c|c|c|c|c|}
\hline Category & \multicolumn{2}{|l|}{1} & \multicolumn{2}{|l|}{$n$} & \multicolumn{2}{|l|}{ W } \\
\hline $\begin{array}{l}\text { information from } \\
\text { number of questions } \\
\text { number of test persons }\end{array}$ & \multicolumn{2}{|c|}{$\begin{array}{l}\text { past history } \\
3 \\
24\end{array}$} & \multicolumn{2}{|c|}{$\begin{array}{l}\text { progress notes } \\
11 \\
36\end{array}$} & \multicolumn{2}{|c|}{$\begin{array}{l}\text { medical history } \\
\text { physical examination } \\
5 \\
24\end{array}$} \\
\hline Medical-record type & average & SE mean & average & SE mean & average & SEmean \\
\hline $\begin{array}{l}\mathrm{A} \\
\mathrm{B} \\
\mathrm{C}\end{array}$ & $\begin{array}{l}90 \\
77 \\
76\end{array}$ & $\begin{array}{l}11.0 \\
9.0 \\
8.4\end{array}$ & $\begin{array}{l}127 \\
101 \\
104\end{array}$ & $\begin{array}{l}14.2 \\
10.5 \\
10.5\end{array}$ & $\begin{array}{l}169 \\
151 \\
174\end{array}$ & $\begin{array}{l}25.7 \\
21.4 \\
19.8\end{array}$ \\
\hline Medical-record type & $p-v$ & & & alue & $p-w$ & \\
\hline $\begin{array}{l}A-B \\
A-C \\
B-C\end{array}$ & $\begin{array}{l}0.3 \\
0.0 \\
0.3\end{array}$ & & $\begin{array}{l}0.0 \\
0.0 \\
0.8\end{array}$ & $\begin{array}{l}00 * * * \\
0 * * * \\
3\end{array}$ & $\begin{array}{l}0.0 \\
0.2 \\
0.0\end{array}$ & \\
\hline
\end{tabular}

* = significant $<0.017 ; \quad * *=$ significant $<0.0017$

$\mathrm{SE}$ mean $=$ standard error of the mean

\subsubsection{Sub-analysis: searching different kinds of information}

We conducted a sub-analysis on categories of questions to to investigate whether differences in outcome can be attributed to a different granularity of medical history and physical examination, to a different granularity of progress notes, or to both (Table 7-14, upper part). Since the main analysis only demonstrated a significant effect of the medical-record type on the speed of information retrieval, we restricted the subanallysis to this outcome measure.

The middle part of Table 7-14 shows the average time, for each medical-record type, a person needed to answer a question in one of the given categories. Searching in problem-partitioned progress notes was between 23 and 26 seconds (about $22 \%$ ) faster than searching in undivided progress notes. Searching medical-history or physicalexamination information in sections divided into organ systems, was 18 seconds $(\| 1 \%)$ faster than searching such information in undivided parts and 23 seconds (13\%) faster than searching such information in sections of single observations. Analysis of variance demonstrates that, indeed, the category of questions (and hence the section of the medical record used) was a significant modifier of the effect the medical recordtype had on the speed of information retrieval $(F=2.86, p=0.023)$.

The lower part of Table 7-14 shows, for each category of questions, whether the differences in speed among the medical-record types were significant (pair-wise t-test). 
The level of significance was 0.017 (Bonferroni). A significant difference in speed was demonstrated between undivided progress notes ( $A$ and $B$ ) on one hand and problempartitioned progress notes $(C)$ on the other (in both cases $p=0.000$ ). There was also a significant difference between medical history and physical examination with paragraphs on the level of organ systems (B) and with paragraphs of single observations (C) $(\mathrm{p}=0.004)$. The difference found between medical history and physical examination as undivided sections (A) and with paragraphs on the level of organ systems (B) was not significant, however.

\subsection{Discussion}

From our study the following conclusions can be drawn. These conclusions should be considered within the restrictions dictated by the validity of our study, which will be discussed in 7.5.3.

Searching information in an electronic medical-record system with undivided medical narratives takes more time than with medical narratives divided into paragraphs. In particular, searching in problem-partitioned progress notes is significantly faster than in undivided progress notes. On the other hand, a too detailed partitioning has also a negative effect on the speed of searching. In particular, searching in medical history and physical examination with paragraphs on the level of single questions and observations is slower than when using paragraphs on the level of organ systems. The times gained when using the fastest structures were, approximately, between 20 to 30 seconds ( 15 to $20 \%$ ) per question, which is certainly worthwhile.

The superiority of problem-partitioned progress notes supports Weed's ideas of how to organise the medical record. ${ }^{28}$ Especially concenning patients with multiple pathology (as we had selected for the test), a division of progress notes into problem paragraphs helps the physician with focusing on one problem at a time, without neglecting the overview. The negative effect of a too detailed partitioning of medical history and physical examination may be due to several causes. The selection of relevant paragraphs may take more time because the number of paragraphs is larger. Another possibility is that the content of a single paragraph becomes so restricted that it does not cover any more the clinical context of the information needed.

The fact that the series of presentation had a significant effect on the speed of information retrieval suggests that the learning effect was still present during the test. Regarding the short learning period at the beginning of the test this is not surprising. We expect that physicians experienced with the system will search faster, but we have no indication to suspect that this will influence the differences between medical-record types.

Searching in the medical record of patient (z) was far more time-consuming and was performed far less completely than searching in the medical record of the other patients. Analysis on the question level indicated that about patient (z) only complex questions were asked, while about the other patients a mixture of simple and more 
complex questions was asked. It could not be demonstrated, however, that the effect of the medical-record type on either of the outcome measures was case dependent.

We did not find evidence for our assumption that specialists perform better with another medical-record type than residents do. Although we cannot exclude that this lack of evidence is a false-negative finding (power $=0.42$ ), it might suggests that a different strategy of thinking does not necessarily result in a different strategy of searching.

\subsection{Granularity and speed of information retrieval: related literature}

A comparative time study to the use of different granularities in electronic medicalrecord systems, as presented here, was never performed. A comparable study using paper-based medical records was performed by Fries in 1974. He compared the speed of information retrieval among physicians when using time-oriented, traditional (source-oriented), and problem-oriented medical records. ${ }^{30}$ In the time-oriented medical record Fries had employed the principle of flowsheet presentation covering the entire medical record. All observations from medical history and physical examination were presented in coded format, leaving only assessment data recorded as free text. Twenty-six test persons had to answer "five common clinical questions" using a number of actual clinical records of similar complexity. These questions concerned measurements of blood pressure $(2 \mathrm{x})$, body weight, medication supply, and one result of a laboratory test. Fries studied the time needed to find the answers. Consulting the time-oriented medical record was, on average, four times faster than using the other record structures.

We share Fries' conclusion that the traditional medical record is the less suitable structure for searching. Other comparisons with Fries' study fall short because both studies differ in several respects. First, Fries compared paper medical records instead of electronic ones. Second, Fries did not include a medical record with intermediate granularity in his study, as we did. Third, Fries presented medical narratives in coded format and not in natural prose. The fourth, and perhaps most important, difference is the fact that Fries" "five common questions" concerned only quantitative information not included in the narrative parts of the medical-record types we used. We presented blood pressure and body weight in the section vital body functions that was identical in all three record types. Also the sections that include medication and laboratory results were equal in all three record types.

\subsubsection{Completeness of retrieved information: related literature}

In the laboratory setting of our test, the physicians were in the position to complete all questions, whatever time it took. Theoretically, we could have expected an average completeness of retrieved information (recall) that approxinates $100 \%$. Compared to our gold standard we found only $80 \%$.

Detailed analysis on the question level showed that nine questions were answered with an average completeness of more than $90 \%$ and the other ten with an average completeness between $50 \%$ and $80 \%$. During the test, the suspicion was raised that six of the less completely answered questions were interpreted wrongly by some test persons (i.e., question $2,3,8,14,15$, and 17 , see Table 7-4). We explored the conse- 
quences of this ambiguous interpretation by repeating the analysis of variance when leaving out these "suspicious" questions. Thus, we found averages of completeness. around $90 \%$ for each medical-record type. Hence, part of the incompleteness can be explained by ambiguities in questions not observed during the validation of the questions (see 7.3.10). However, also in this additional exploration we did not find evidence of a record-type effect on the completeness of retrieved information $(p=0.247)$.

A study of a different kind but also in a simulative setting was conducted by Hripcsak et al. ${ }^{37}$ They studied the completeness of information as found by seven physicians answering eight questions about laboratory results when using two alternative search tools: a sophisticated browser that supports searching in various ways (lexical matching, semantic links, etc.) and a much simpler browser based on the hierarchic, source-oriented, organisation of patient records as used in the hospitalinformation system. Compared with a gold standard, they found $68 \%$ recall when using the sophisticated browser and $74 \%$ when using the simpler browser. Since the area of medical narratives is likely to be much fuzzier than the laboratory domain, the average of $80 \%$ we found seems reasonable. Hripcsak at al. blamed the poor performance they found to the fact that, perhaps, their gold standard was too harsh. Maybe this is also the case on our study. However, in both studies the central issue was not the absolute search performance of physicians, but the differences between alternative ways of retrieving information.

In our study the physicians answered all questions satisfactorily, according to their own opinion. In daily practice, however, physicians often work under time pressure, which may lead to less complete answers when searching takes too much time. Tang et al. studied the completeness of patient information retriewed by physicians during 168 return outpatient visits. ${ }^{38}$ In $8 \rrbracket \%$ of these visits, in which the physicians could use both the hospital-information system and a paper shadow record, the physicians could not find all patient information needed, according to their own opinion. Of the data missing, 31\% was related to medical narratives. Tang's study supports our hypothesis that the completeness of retrieved information will decrease when physicians have to search for information in a limited period of time. It is likely that this effect will occur in the slowest searching structure first.

\subsubsection{Limitarions of the study}

The fact that our test was performed in a simulative, laboratory-like, setting enabled the elimination of many disturbing factors but at the same time limits the generalisability of our findings. We already mentioned the absence of time pressure, but there are other factors not controlled in a real-life setting.

For example, we only used voluminous cases. When retrieving information from smaller cases, it can be expected that searching in undivided medical narratives will becone less problematic. Another factor controlled in our test was the absent influence of the physician's memory. In routine medical practice, a part of the information is memorised by the physician, thus reducing the need to consult the medical record. In addition, the medical record may also serve as a trigger for the physician's memory so 
that he will also remember other information again. ${ }^{31}$ it is impossible to estimate the effect of memorisation on the granularity of the medical record.

The reasons for consulting and questions put forward in our study were only representative for the cases used. We tried to enhance the coverage of our question mixture by instructing the author to keep in mind the indications for reading found by Nygren and Henrikson. ${ }^{3}$ More specific instructions could have resulted in a better mixture but would also have held the risk of violating the blindness of the author. More objective measures for the search-technical complexity of a question are not available, so an in-vitro selection can be at best illustrative, but never representative.

We concluded that problem partitioning leads to faster searching. However, a limitation of the medical-record types we used was the fact that we did not include the process view as part of the searching structure. (It was difficult and hazardous to reconstruct a process view from the existing cases used and, besides, we did not want to interfere with the test persons' view of the process based on the medical data only.) Problem view and process view are both selective views: the problem view presents a selection of medical data types that may be relevant in the context of a given problem, while the process view points to medical data that were acmally used when taking a particular decision. In other words, the problem view pre-empls possible process views. Hence, the value of the problem view can be questioned when reviewing information in a medical record that also provides a process view.

Also the electronic medical-record system we used had some limitations that could restrict the validity of our conclusions. The software-development kit we used (Visua) Basic $3.0^{\mathrm{rm}}$ ) did not support features for free-text searching. For example, within the text window it was not possible to search by keywords. Free-text searching by keywords (by the use of so-called search engines) has become popular with the growing use of World-Wide-Web browsers. For searching in medical narratives with coarse granularity it will certainly have an added value. On the other hand, the added value of search engines should not be overestimated. When reading medical natratives, also the clinical context of medical data has a guiding role. Search engines do not take this context into account, which may lead to an erratic way of searching.

The best way to validate our conclusions under a rich variety of circumstances is to perform a similar study in daily medical practice. For such a study we need physicians that routinely use the electronic medical record for consulting all patient medical data needed, including medical narratives. At present, such systems do not exist, at least not in The Netherlands.

\subsubsection{Implications for arher medical domains}

We studied the retrieval of information of medical narratives within the domain of internal medicine of an academic hospital. Which significance has our study for other disciplines and settings?

It is often mentioned that each discipline imposes its own requirements to the structure of the medical record. For example, Van Ginneken and Stam suggested to split the medical record into a mother record suitable for every discipline and a variety of sub-records (supported by domain-specific knowledge bases of medical terms) in which each specialty can accommodate its own structure. ${ }^{25}$ The optimall structure we 
found had a granularity of medical history and physical examination on the level of organ systems. Such a structure is general for any medical discipline, hence concerning Van Ginneken's mother record rather than a sub-record. Further support for the generalisability of this granularity to other medical domains comes from Nygren et al. They observed the way of reading of physicians from different specialties and concluded that retrieving information from the medical record is a general skill which is an integrated part of medical expertise. ${ }^{31}$ Based on these observations, they arrived at an electronic medical-record system with free-text medical narratives divided into paragraphs on the level of organ systems, just as we did. ${ }^{26}$

We also see no reason to believe that physicians in non-academic hospitals search differently than their colleagues in academic settings. However, anticipating a weak response of non-academic physicians to participate in a simulation study, we believe a validation of our findings in such a setting will be only feasible when the study can be carried out in real-life practice.

\subsubsection{Free text or structured data?}

Natural language is the typical means used to communicate between human beings. It gives the author the flexibility needed to express all nuances of his observations and thoughts, and gives the reader the contextual information needed to get an impression of the medical-care process. Main shortcoming of free-text medical narratives is the little added value of computers over paper when processing such information. This shortcoming concerns the storage of medical narratives, not the presentation. We presented medical narratives as free text because it is the preferred presentation format in many electronic medical-record systems, even if the prose has to be generated from coded data., ${ }^{4}, 12.14,39-41$

However, it has never been proven that free-text presentation of medical narratives facilitates information retrieval more than presentation in a structured format. Arguments in favour of presenting medical narratives as free text are the ease of reading and the embedding of data in their clinical context. Main argument in favour of presenting medical narratives in structured format (as controlled expressions) is the possibility of using SQL-like queries when searching specific data. On the other hand, presenting structured medical data in structured format implies partitioning them into the most detailed level possible: the level of separate attributes (which is finer than the finest granularity in our study). We found, however, that dividing medical narratives into too detailed paragraphs slows down the retrieval of information. We already suggested that this could be due to incomplete coverage of the clinical context. This argument remains in full force when medical narratives in structured format are concerned. Hence, we do not expect that the advantages of presenting structured data will outweigh those of free-text paragraphs on the level of organ systems.

Choosing for a free-text presentation of medical narratives does not prevent, however, from storing these data in structured format as controlled expressions. In our system, we stored medical narratives as free text (with the same granularity as they are presented) only for reasons of convenience. When using an electronic medical-record system, the format in which medical narratives are presented is, in principle, independent from the way they are entered and stored. Conversion from structured data 
to natural prose needs the application of a natural-language generator. The literature reveals a long history of experience with natural-language generation in a broad domain of application. ${ }^{42,12,39-41}$ Thus, it seems very well possible to combine structured storage of medical narratives with free-text presentation, collated to a granularity of choice, e.g., on the intermediate level.

\subsection{Conclusion}

Electronic medical-record systems may facilitate the retrieval of information to support direct patient care. Existing systems present their narratives in different granularities. Most benefit can be expected from paragraphing medical history and physical examination on the level of organ systems, and partitioning progress notes in problem sections. This conclusion has been obtained via a simulation study with large and complex patient cases only. Further study, in real-life practice, is needed to validate our conclusions under other circumstances, such as searching under time pressure, searching in smaller cases, searching information that is partly memorised, searching with the availability of full-text search engines, and searching in a medical record that also provides a process view.

\subsection{References}

1. Dick RS, Stein EB. The computer-based patient record - an essential technology for health care. Washington DC: National Academy Press, 1991.

2. McDonald CJ, Blevins L, Tierney WM, Martin DK. The Regenstrief medical records, MD Comput 1988; 5:34-47.

3. Whiting O'Keefe QE, Whiting A, Henke J. The STOR clinical information system. MD Comput $1988 ; 5: 8-21$.

4. Stead WW, Hammond WE. Computer-based medical records: the centerpiece of TMR. MD Comput 1988; 5:48-62.

5. Barnett GO. The application of computer-based medical-record systems in ambulatory practice. N Engl J Med 1984; 310:1643-50.

6. Johnson $K$, Poon A, Shiffman S, Lin R, Fagan L. A history-taking system that uses continuous speech recognition. Proc Annu Symp Comput Appl Med Care 1992.757-61.

7. Rassinoux AM, Wagner $₫ C$, Lovis C, Baud RH, Rector A, Scherrer JR. Analysis of medical texts based on a sound medical model. Proc Annu Symp Comput Appl Med Care 1995:27.31.

8. Sager $N_{,}$Lyman $M$, Bucknall $C$, Nhan $N$, Tick LJ. Natural language processing and the representation of clinical data. I Am Med Inform Assoc 1994; 1:142-60.

9. Heathfield HA, Kirby J, Hardiker NR. Data entry in computer-based care planning. Comput Methods Programs Biomed 1995; 48:103-107.

10. Poon AD, Fagan LM. PEN-Ivory: the design and evaluation of a pen-based computer systen for structured data entry. Proc Annu Symp Comput Appl Med Care 1994:447-51.

11. Huff SM, Pryor TA, Tebbs RD. Pick from thousands: a collaborative processing model for coded data entry. Proc Annu Symp Comput Appl Med Care 1992:104-8.

12. Gouveia Oliveira A, Raposo VD, Azevedo AP, et al. SISCOPE: a multiuser information system for gastrointestinal endoscopy. Endoscopy 1991;23:272-7,

13. Mooman PW, van Ginneken AM, wan der Lei J, van Bemmel JH. A model for structured data entry based on explicit deseriptional knowledge. Methods Inf Med 1994; 33:454-63.

14. Trace D, Naeymi Rad F, Haines D, et all. Intelligent Medical Record---entry (IMR - E). J Med Syst $1993 ; 17: 139-51$. 
15. Rothwell DJ. SNOMED-based knowledge representation. Methods Inf Med 1995; 34:209-157.

16. Rector AL, Solomon WS, Nowlan WA, et al. A terminollogy server for medical language and medical information systems. Methods luf Med 1995; 34:147-157.

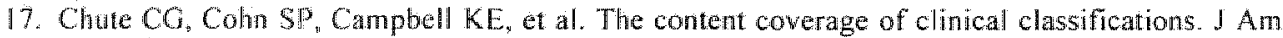
Med Inform Assoc 1996; 3:224-233.

18. Barnett $\mathrm{GO}$, Jenders $\mathbb{R A}$, Chueh $\mathrm{HC}$. The computer-based clinical record-where do we stand? [editorial]. Anm Intern Med 1993; 119:1046m8.

19. McDonald CI, Tierney WM. Computer-stored medical records. Their future role in medical practice. Jama 1988; 259:3433-40.

20. McDonald CJ, Banett GO. Medical-record systems. In: Shortliffe EH, Perreault LE, eds. Medical Informatics - Computer Applications in Health Care. Reading MA: Addison-Wesley, $1990: 181-218$

21. Bolens $M$, Borst $\mathbb{F}$, Scherrer JR. Organizing the clinical data in the medical record. MD Comput $1992 ; 9: 149-55$

22. Fries JF. Time-oriented patient records and a computer databank. Jama $1972 ; 222: 1536-42$.

23. Stead WW, Hammond WE, Straube MJ. A chartless record--is it adequate? J Med Syst 1983; 7:103.9.

24. Safran C, Porter D, Lightfoot $J$, et al. ClinQuery: a system for online searching of data in a teaching hospital. Ann Intern Med 1989; 111:751-6.

25. wan Ginneken AM, Stam H. Can one patient record accommodate the diversity of specialized care'? Proc Annu Symp Comput Appl Med Care 1995:406-10.

26. Nygren $E$, Johnson $M$, Henriksson P. Reading the medical record. II. Design of a humancomputer interface for basic reading of computerized medical records. Comput Methods Programs Biomed 1992; 39:13-25.

27. Heathineld HA, Hardiker N, Kirby J, Tallis R, Gonsalkarale M. The PEN \& PAD medical record model: development of a nursing record for hospital-based care of the elderly. Methods Inf Med 1994; 33:464-72.

28. Weed LL. Medical records that guide and teach. N Engl J Med 1968; 278:593-600, 652-657.

29. de Vries Robbé PF. Useful elements of P.O.M.R. - a report of experience. Medical Record 1975; 16:13-17.

30. Fries JF. Alternatives in medical record formats. Med Care 1974; 12:871-81.

31. Nygren $E$, Henriksson $\mathbb{P}$. Reading the medical record. 1. Analysis of physicians' ways of reading the medical record. Comput Methods Programs Biomed 1992; 39:1-12.

32. Custers EIFM, Boshuizen HPA. Schmidt HG. The influence of medical expertise case typicality. and illness script component on case processing and disease probability estimates. Memory \& Cognition 1996; $24: 384-399$.

33. Senn S. Cross-over trials in clinical research. Chicester: John Wiley \& Sons Lid, 1993.

34. Jones B, Kenward MG. Design and analysis of cross-over trials. London: Chapman and Hall, 1989.340 .

35. Norusis MJ. SPSS Adwanced statistics 6.1. Chicago, IL: SPSS Inc, 1994.

36. Altman DG. Practical statistics for medical researeh. London: Chapman and Hall, 1991:611.

37. Hripcsak G, Allen B, Cimino JJ, Lee $\mathbb{R}$. Access to data: comparing AccessMed with query by review. J Am Med Inform Assoc 1996; 3:288-299.

38. Tang PC, Fafchamps D, Shortliffe EH. Traditional medical records as a source of clinical data in the outpatient setting. Proc Annu Symp Comput Appl Med Care 1994:575-9.

39. McDonald $\mathrm{Cl}$, Tierney WM, Blevins $L$. The benifits of automated medical record systems for ambulatory care. In: Orther HF, Blum Bl, eds. Inplementing health care information systems. New York: Springer-Verlag, 1988 .

40. Poon AD, Fagan LM, Shortliffe EH. The PEN-Ivory project: Exploring user-interface design for the selection of items from large controlled vocabularies in medicine. J Am Med Inform Assoc $1996 ; 3: 168-183$.

41. Bermauer J. Conceptual graphs as an operational model for descriptive findings. Proc Annu Symp Comput Appl Med Care 1991:2 $14-8$. 


\section{Appendix}

Medical-narrative structure of the medical-record types studied in Chapter 7. Paragraphs are formulated in Dutch.

\subsection{Source view}

Hierarchical list of paragraphs, organised according to the type of medical data. Only narrative paragraphs are listed.

\subsection{1 coarse-granularity paragraph}

intermediate-gramularity paragraph

fine-granularity paragraph

fine-granularity paragraph 


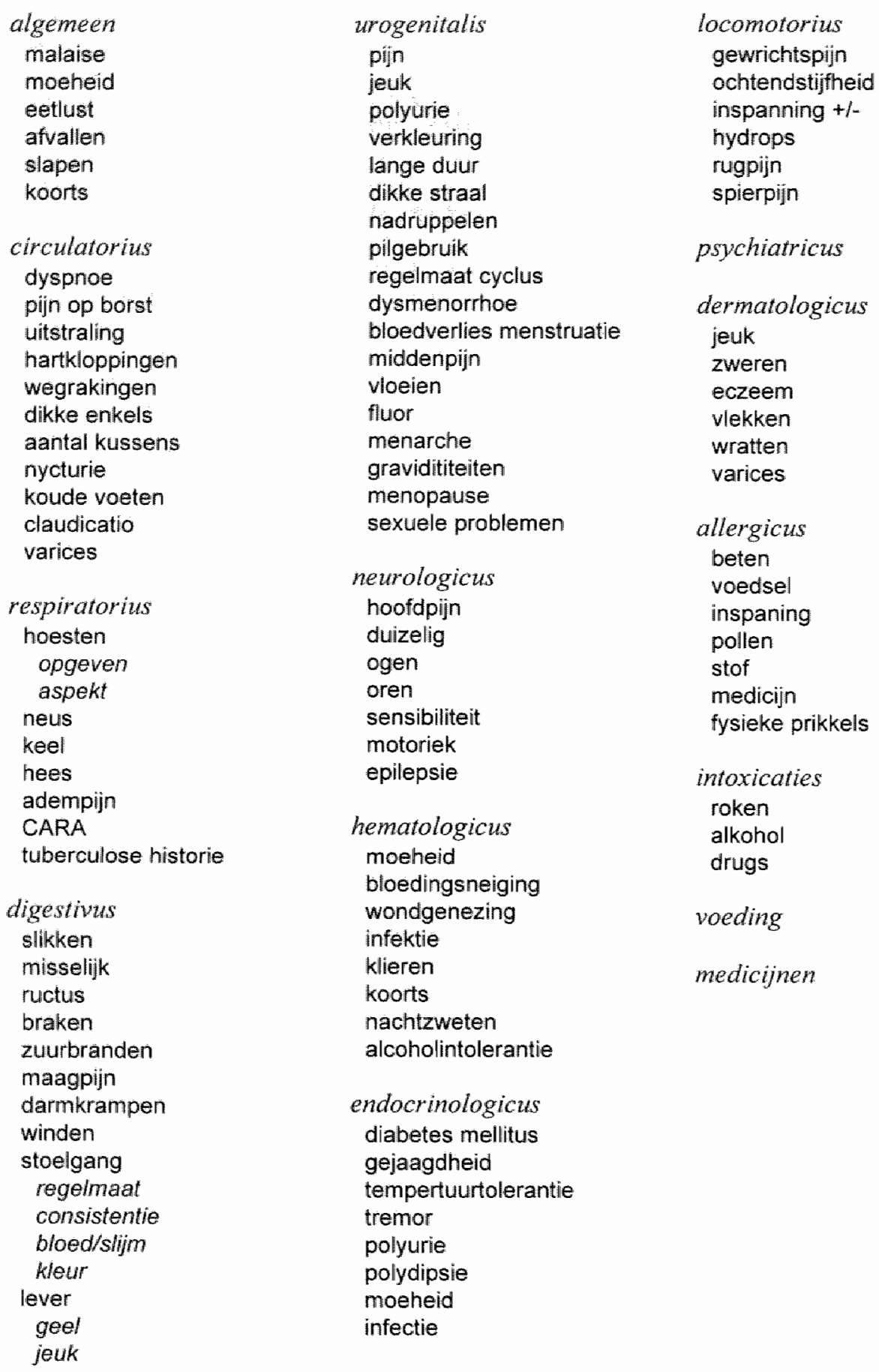




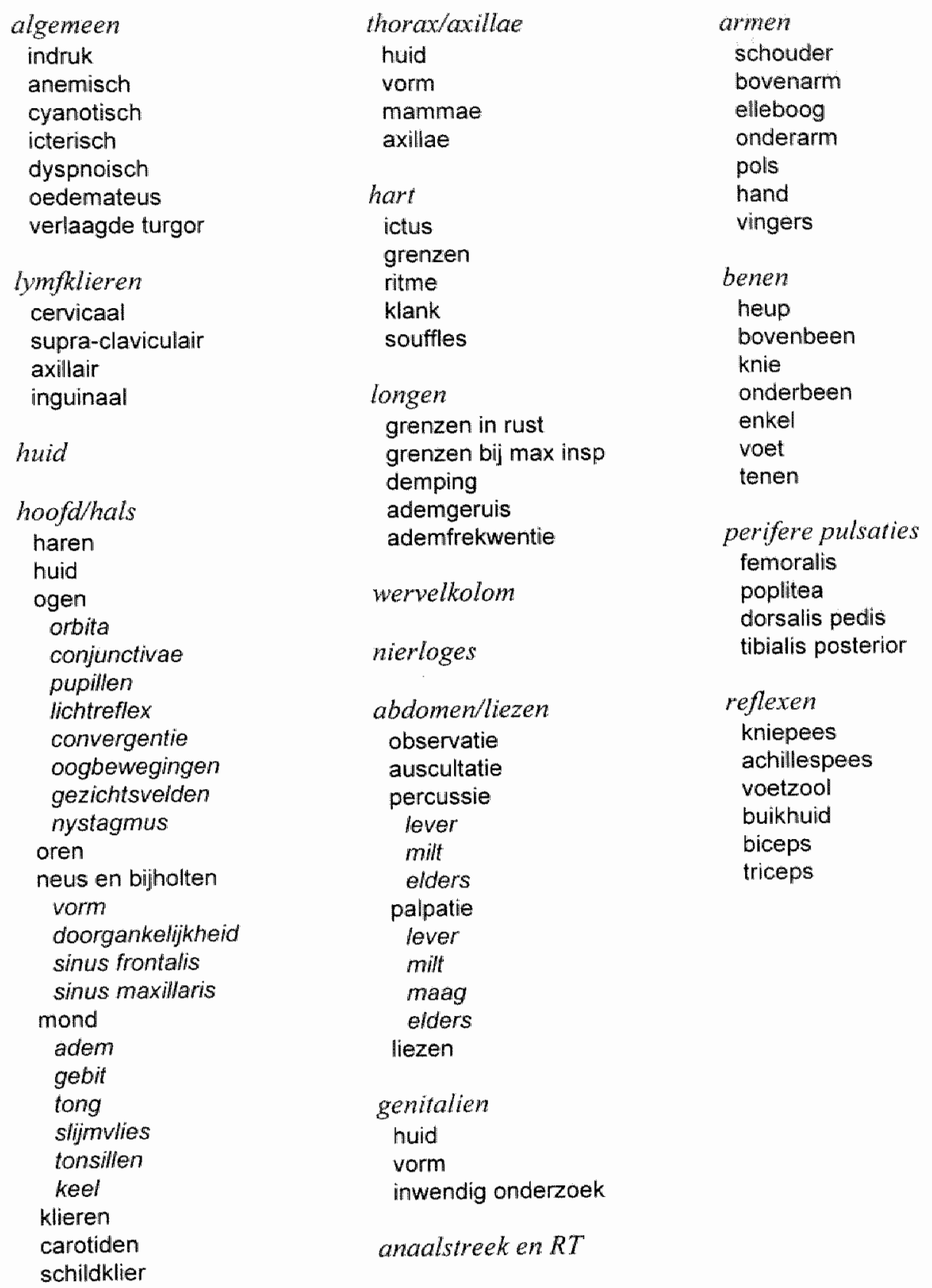


algemene toestand

abmt bij hodgkin

hickmann-catheter

hoesten

koorts e.c.i.

exantheem

verminderde eellust

pancytopenie

stomatitis

afwijking $x$-thorax

alcohol-abusus

zwelling onderbeen $l i$

levercirrhose

nierinsufficientie

anemie

tophenze jicht

verzorgings-probleem

hernia umbilicalis

hypothermie e.c.i.

peri-anaal abces

decompensatio cordis

nien-dialyse

verdenking leukemie chemo bij ac. muel.

leuk.

dyspnoe

vochretentie

trombopenie

leukopenie

ulcus cruris links

hypokaliemie

latente diab. mell. 


\subsection{Problem view}

List of problems used, in combination with the paragraphs that can be relevant for the given problem. Only narrative paragraphs are listed. Example with the intermedtate medical-record type (paragraphs on the level of organ systems).

\begin{tabular}{|c|c|c|}
\hline Problem & Relevant narrative paragraphs & Further problem link \\
\hline ABMT BIJ HODGKIN & Lo/lymfomen & \\
\hline ABMT BIJ HODGKIN & problemen & HICKMANN-CATHETER \\
\hline ABMT BIJ HODGKIN & problemen & ANEMIE \\
\hline ABMT BUJ HODGKIN & problemen & TROMBOPENIE \\
\hline ABMT BIJ HODGKIN & problemen & LEUKOPENE \\
\hline $\begin{array}{l}\text { AFWIJKING X-THORAX } \\
\text { AFWIJKING X-THORAX }\end{array}$ & $\begin{array}{l}\text { A/tractus respiratorius } \\
\text { LOllongen }\end{array}$ & \\
\hline ALCOHOL-ABUSUS & Avtractus digestivuis & \\
\hline ALCOHOL-ABUSUS & Avtractus neurologicus & \\
\hline ALCOHOL-ABUSUS & Altractus psychiatricus & \\
\hline ALCOHOL-ABUSUIS & LOlabdomen & \\
\hline ALCOHOL-ABUSUS & $\begin{array}{l}\text { LOlalgemene indruk } \\
\text { gezondheidstoestand }\end{array}$ & \\
\hline ALCOHOL-ABUSUS & problemen & LEVERCIRRHOSE \\
\hline ANEMIE & Avtractus algemeen & \\
\hline ANEMIE & Avtractus digestiwus & \\
\hline ANEMIE & Avtractus hematologicus & \\
\hline ANEMIE & Avoeding & \\
\hline ANEMIE & $\begin{array}{l}\text { LO/algemene indruk } \\
\text { gezondheidstoestand }\end{array}$ & \\
\hline ANEMIE & LO/anaalstreek en rectaal toucher & \\
\hline ANEMIE & problemen & TROMBOPENIE \\
\hline ANEMIE & problemen & LEUKOPENIE \\
\hline CHEMO BIJ AC. MYEL. LEUK. & A/tractus algemeen & \\
\hline CHEMO BIJAC MYEL. LEUK. & A/tractus hematologicus & \\
\hline CHEMO BIJ AC MYEL. LEUK. & LO/abdomen & \\
\hline CHEMO BIJ AC. MYEL. LEUK. & $\begin{array}{l}\text { LO/aigemene indruk } \\
\text { gezondheidstoestand }\end{array}$ & \\
\hline CHEMO BUJ AC. MYEL. LEUK. & Lo/hoofd en halsgebied & \\
\hline CHEMO BIJ AC. MYEL LEUK. & Lollymfomen & \\
\hline CHEMO BIJ AC MYEL. LEUK. & problemen & LEUKOPENIE \\
\hline CHEMO BIJ AC MYEL LEUK. & problemen & TROMBOPENIE \\
\hline CHEMO BIJ AC. MYEL. LEUK. & problemen & ANEME \\
\hline CHEMO BIJ AC MYEL LEUK. & problemen & HICKMANN-CATHETER \\
\hline DYSPNOE & Avtractus circulatorius & \\
\hline DYSPNOE & A/tractus respiratorius & \\
\hline DYSPNOE & Lo/hart & \\
\hline DYSPNOE & Lollongen & \\
\hline DYSPNOE & problemen & AINEMIE \\
\hline EXANTHEEM & A/tractus dermatologicus & \\
\hline EXANTHEEM & LO/hoofd en halsgebied & \\
\hline EXANTHEEM & LO/huid & \\
\hline
\end{tabular}




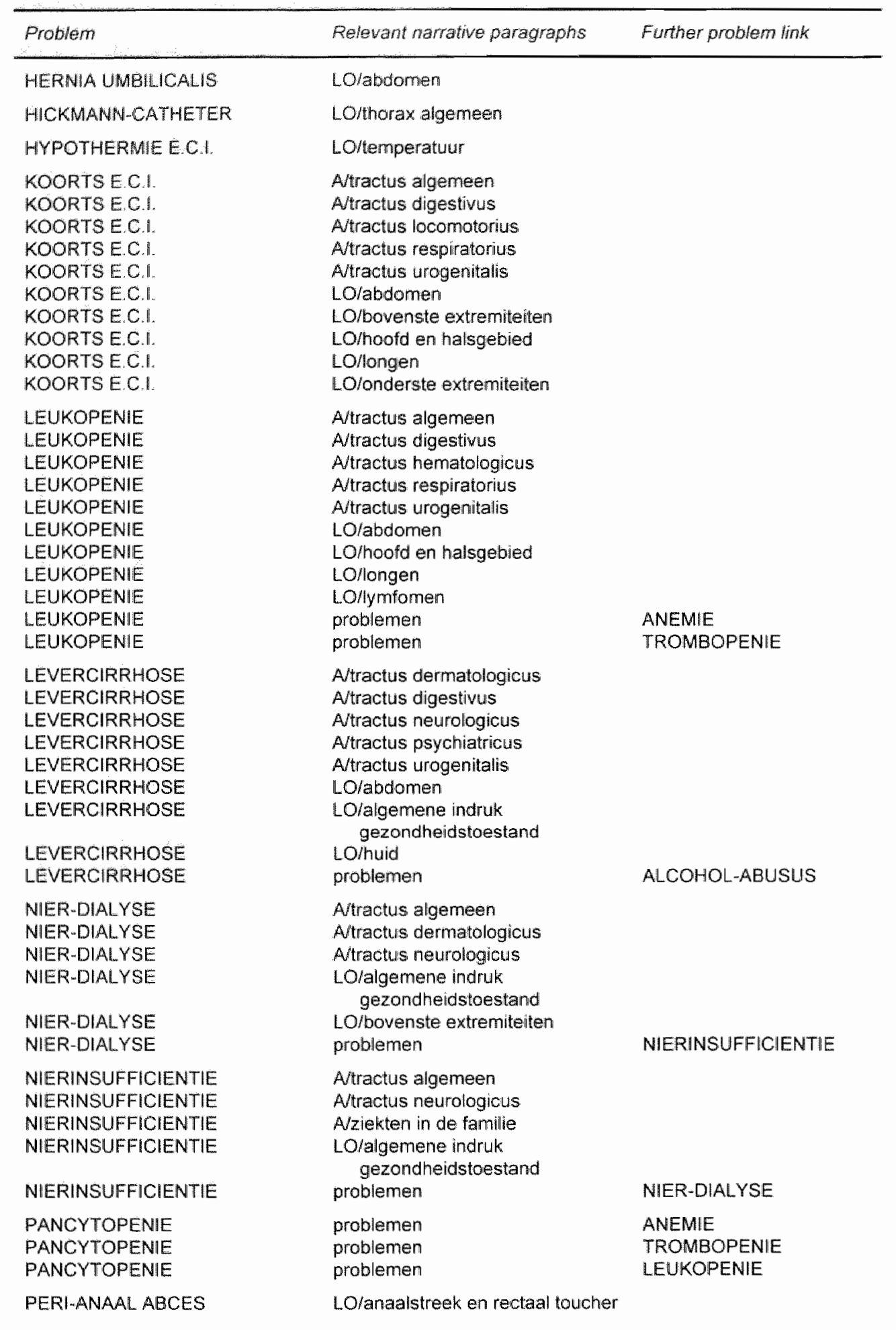




\begin{tabular}{|c|c|c|}
\hline Problem & Relevant narrative paragraphs & Further problem link \\
\hline $\begin{array}{l}\text { PHARYNGITIS } \\
\text { PHARYNGITIS } \\
\text { PHARYNGITIS }\end{array}$ & $\begin{array}{l}\text { Altractus respiratorius } \\
\text { LO/hoofd en halsgebied } \\
\text { Lo/lymfomen }\end{array}$ & \\
\hline $\begin{array}{l}\text { STOMATITIS } \\
\text { STOMATITIS } \\
\text { STOMATITIS }\end{array}$ & $\begin{array}{l}\text { A/tractus digestivus } \\
\text { A/tractus irespiratorius } \\
\text { LO/hoofd en halsgebied }\end{array}$ & \\
\hline $\begin{array}{l}\text { TOPHEUZE JICHT } \\
\text { TOPHEUZE JICHT } \\
\text { TOPHEUZE JICHT } \\
\text { TOPHEUZE ЛICHT } \\
\text { TOPHEUZE JICHT }\end{array}$ & $\begin{array}{l}\text { A/tractus locomotorius } \\
\text { LO/bovenste extremiteiten } \\
\text { LO/hoofd en halsgebied } \\
\text { LO/onderste extremiteiten } \\
\text { problemen }\end{array}$ & NIERINSUFFICIENTIE \\
\hline $\begin{array}{l}\text { TROMBOPENIE } \\
\text { TROMBOPENIE } \\
\text { TROMBOPENIE } \\
\text { TROMBOPENIE } \\
\text { TROMBOPENIE }\end{array}$ & $\begin{array}{l}\text { A/tractus digestivus } \\
\text { A/tractus hematologicus } \\
\text { A/tractus urogenitalis } \\
\text { LO/huid } \\
\text { problemen }\end{array}$ & ANEMIE \\
\hline ULCUS CRURIS LINKS & LO/onderste extremiteiten & \\
\hline $\begin{array}{l}\text { VERDENKING LEUKEMIE } \\
\text { VERDENKING LEUKEMIE } \\
\text { VERDENKINIG LEUKEMIE } \\
\text { VERDENKING LEUKEMIE }\end{array}$ & $\begin{array}{l}\text { A/tractus algemeen } \\
\text { Atractus hematologicus } \\
\text { LOlabdomen } \\
\text { LO/algemene indruk } \\
\quad \text { gezondheidstoestand }\end{array}$ & \\
\hline $\begin{array}{l}\text { VERDENKING LEUKEMIE } \\
\text { VERDENKING LEUKEMIE } \\
\text { VERDENKING LEUKEMIE } \\
\text { VERDENKING LEUKEMIE } \\
\text { VERDENKING LEUKEMIE }\end{array}$ & $\begin{array}{l}\text { LO/hoofd en halsgebied } \\
\text { LO/lymfomen } \\
\text { problemen } \\
\text { problemen } \\
\text { problemen }\end{array}$ & $\begin{array}{l}\text { ANEMHE } \\
\text { TROMBOPENIE } \\
\text { LEUKOPENIE }\end{array}$ \\
\hline VERMINDERDE EETLUST & Avtractus algemeen & \\
\hline $\begin{array}{l}\text { VERZORGINGS-PROBLEEM } \\
\text { VERZORGINGS-PROBLEEM }\end{array}$ & $\begin{array}{l}\text { A/tractus psychiatricus } \\
\text { problemen }\end{array}$ & ALCOHOL-ABUSUS \\
\hline $\begin{array}{l}\text { VOCHTRETENTIE } \\
\text { VOCHTRETENTIE } \\
\text { VOCHTRETENTIE } \\
\text { VOCHTRETENTIE } \\
\text { VOCHTRETENTIE } \\
\text { VOCHTRETENTIE }\end{array}$ & $\begin{array}{l}\text { A/traclus circulatorius } \\
\text { Atractus respiratorius } \\
\text { LO/algemene indruk } \\
\text { gezondheid stoestand } \\
\text { LO/hart } \\
\text { LO/longen } \\
\text { problemen }\end{array}$ & HYPOKALIEMIE \\
\hline $\begin{array}{l}\text { ZWELLING ONDERBEEN LI } \\
\text { ZWELLING ONDERBEEN LI } \\
\text { ZWELLING ONDERBEEN LI } \\
\text { ZWELLING ONDERBEEN L }\end{array}$ & $\begin{array}{l}\text { A/lractus circulatorius } \\
\text { A/tractus locomotorius } \\
\text { Altractus respiratorius } \\
\text { LOlalgemene indruk } \\
\quad \text { gezondheidstoestand }\end{array}$ & \\
\hline $\begin{array}{l}\text { ZWELLING ONDERBEEN LI } \\
\text { ZWELLING ONDERBEEN LI } \\
\text { ZWELLING ONDERBEEN LI } \\
\text { ZWELLING ONDERBEEN LI } \\
\text { ZWELLING ONDERBEEN LI }\end{array}$ & $\begin{array}{l}\text { LO/hart } \\
\text { LO/longen } \\
\text { LO/lymfomen } \\
\text { LO/onderste extremiteiten } \\
\text { LO/temperatuur }\end{array}$ & \\
\hline
\end{tabular}





\section{Summary, conclusions, and discussion}

\subsection{Summary and conclusions}

All medical-care activities involve gathering, analysing, or using medical data. The principle repository of medical data about a single patient is the medical record. The primary function of the medical record is to provide the physician with information for direct medical practice, originally as an aide memoir for the individual physician, but more and more as a multi-authored communication tool for physicians and other professionals sharing the responsibility for individual-patient care.

\subsubsection{Users about the paper medical record}

In the field of medical informatics, it is generally recognised that the paper-based medical record has become obsolete. Indeed, as a provider of management information, as a source for research, or for other secondary purposes, the advantages of electronic medical-records systems are unquestionable. With respect to its primary use in direct medical practice, however, physicians seem still to appreciate their "goodold" paper medical record. In a survey among eighty-five hospital physicians, we found that they especially appreciated the flexible data-entry facilities of the paper medical record and that they considered its content reliable enough to work with. On the other hand, also some shortcomings of the paper-based medical record were indicated in this survey: the retrieval of information should be faster, the registration of decisions, plans, and orders in the medical record should be more complete, and the security and confidentiality of medical data should be better guaranteed. We learned that we should be careful with projecting conclusions about medical-record keeping, as described in the literature, to a local situation. When considering the introduction of an electronic medical-record system, an assessment of the users' appreciation of the existing paper-based system should always be part of the procedure. Not only the need for improvement but also the appreciated aspects of the current system should be taken into accoumt.

\section{1.2 Problems with medical narratives in electronic medical-record systems}

Apart from this satisfaction with the paper medical record among potential users, also other impeding factors for the development and distribution of electronic medicalrecord systems have been identified in the literature. All these barriers have in common that they mainly pertain to the narrative parts of the medical record, in particular medical history, physical examination, and progress notes. With respect to the management of medical narratives in electronic medical-record systems "three 
challenges for research have been addressed in the literature: how to enhance the understandability of data (by the use of controlled terminologies), how to facilitate the entry of data, and how to facilitate the retrieval of information

The last challenge, information retrieval from medical narratives, can be considered as a two-step process. First, the user has to search through the medical record to select the paragraph(s) that contain relevant information. This step can be facilitated by structuring medical narratives in a way that guides the user to his target. Second, the user has to read the content of the selected paragraph(s) to retrieve the information needed. The content of a medical-narrative paragraph is usually presented as free text.

\subsubsection{A multiple-view searching structure}

To provide an electronic medical-record system with a flexible searching structure, the traditional source-oriented organisation can be extended with other structuring elements: problem-oriented progress notes (with or without SOAP-structure) and flowsheets that present medical data as a function of time. Source-oriented and problem-oriented flowsheets are commonly used in existing electronic medical-record systems, albeit for non-narrative data only.

We analysed three well-known medical-record types (the source-oriented, problemoriented, and time-oriented medical record) and deduced from them four organising principles, each of which is considered as a "view" on medical data:

- a time view: a logical ordering of medical data according to their time of registration

- a source view: a hierarchical organisation of medical data determined by their type

- a problem view: a selection of medical-data types that can be relevant to a particular medical problem

- a process view: a link between medical data expressing their respective roles in the establishment of medical practice.

We demonstrated that, from a technical point of view, all four views can be combined in one data model and hence can be part of the searching structure of free-text medical narratives.

From a functional point of view, however, the implementation of the process view is problematic. This is probably due to limitations of the human-computer interface in combination with the absence of a systematic registration routine among physicians. But also from a conceptual point of view, the familiar representation of the process view (Weed's "SOAP-structure") has some shortcomings, in particular when used in a setting of shared medical practice. For example, it does not further specify the assessment of medical problems and it cannot represent the role of conversations (Rector"s "clinical dialogue") as part of medical practice. To overcome these conceptual limitations, we introduced the "conversation model" of systematic medical practice. In this model, medical reasoning is dividedl into four levels that interact with one another via conversations. These four levels are: patient-problem solving, medicalproblem solving, hypothesis testing, and medical acting. Typically, the conversation model does not make a distinction between intra-individual and inter-individual conversations, which makes it applicable for both individual and shared medical care. 
Although the conversation model is only a first atrempt to conceptualise systematic shared medical care, we believe it has the potential to serve as a reference framework for various tasks: not only medical-record keeping, but also medical andit, medical education, and the classification of decision-support systems.

\subsubsection{A multiple-view electronic medical-record system}

We have developed an electronic medical-record system specifically to facilitate information retrieval from medical narratives. In this system, which has a simple and stable user interface, we implemented a searching structure consisting of sourceoriented and problem-oriented flowsheets. Both these flowsheets have a hierarchical structure which enables the user to zoom in for obtaining more detailed paragraphs. Selecting an area of a flowsheet gives access to the corresponding paragraph(s) of the medical record which for medical narratives consist(s) of free text. We extended the system with a research module that can guide the user by instructions and that can record each user-driven event.

\subsubsection{Towards an optimal granularity for the searching structure}

A difference between medical narratives and other medical data is, that the latter are entered, stored, and presented as single findings, while narrative expressions are usually collated to a fluent-prose story. A searching structure for an electronic medicalrecord system with multiple views on medical narratives is only possible, however, when this story is divided into indexable paragraphs. The more detailed such paragraphs are defined, the smaller the granularity of the searching structure and the more specific the information of the selected paragraph. The literature reveals that existing electronic medical-record systems present medical narratives in various granularities. Progress notes can be undivided or indexed into problem sections; medical history and physical examination can be undivided, indexed on the level of organ systems, or even indexed as separate observations.

With the electronic medical-record system described above, we studied three different medical-record types to find the optimal granularity for a searching structure that makes use source-oriented and problem-oriented flowsheets. For the purpose of this study we did not make use of the process view. We recorded three original, voluminous, patient cases from internal medicine into all three medical-record types and submitted some specific questions concerning these patients that asked for information from the medical narratives, to thirty-six physicians. We found the fastest information retrieval with medical history and physical examination divided into paragraphs on the level of organ systems and with progress notes divided into problem sections. We concluded that a searching structure within medical narratives facilitates information retrieval, but that the most detailed structure is not necessarily the best.

\subsection{Discussion}

This thesis has left some issues unanswered that may need further research. In this final section we discuss two of them. First, we studied information retrieval from medical narratives only for the purpose of for direct medical practice. What can be said 
about the optimal gramularity of medicall narratives when used for other purposes? Second, we did not include the process view in our experiment. What can be said about the optimal granularity of medical narratives when the process view would be available to the user?

\subsubsection{Medical narratives and purposes other than direct medical practice}

Although we studied the most used function of the medical record, namely the retrieval of information for direct medical practice, this is not its only purpose: the 1991 report of the Institute of Medicine presented an extensive list of possible purposes of the eleetronic medical record.

For reimbursement purposes, the structure of medical narratives is not very relevant since the cost of medical-history taking and physical-examination performing is not dependent on their content. Other functions of the medical record concerning individual patients, like case-bound medical audit, medical education, and accounting for medico-legal purposes, have in common with medical practice the need for considering medical data in relation to their clinical context. Regarding this common characteristic of information needs we assume that these functions impose comparable requirements to an electronic medical-record system as direct medical practice does. The future with physicians routinely using the electronic medical record for medical practice will learn whether further specification of the requirements for each of these purposes is needed.

On the other hand, when using the medical record as a source for clinical research, it is important that clinical data are unambiguously understood. A third category of purposes such as health-care planning, utilisation's review, and medical audit based on case mixes (instead of individual patients), need aggregated information from the medical record, which is only possible, again, if the original clinical data are unambiguously understood, while contextual information is less important. For these purposes free text is a menace; instead, they need medical narratives cut into elementary pieces of single standardised findings.

We believe, however, that demanding from physicians to record information in the explicit format required by these purposes, often additional to their own information needs, is not the appropriate way. The best way will be to extract aggregated information from medical data originally recorded for direct medical practice. Further research is needed concerning alternative strategies to obtain this. One possible strategy would be to support dynamic data entry of medical narratives as controlled expressions, using a completely covering medical terminology, and process these data in two ways. First, for case-bound purposes, they would routinely be collated to the format of indexable paragraphs of free-text prose. Second, for research and case-mixbound purposes, some of these data could be aggregated using SQL-queries (probably extended by special functions, e.g., to discover temporal relations).

\subsubsection{Problems with the process view}

The medical record is more than just the registration of the present state of a patient. It is also the description of how health-care professionals manage the problems they face. Insight in the subjectivity of medical decisions (their indication, their assessment) and 
in the inter-subjectivity of the clinical dialogue is crucial for a proper understanding of how medical practice is delivered. Such understanding is not only the wish of "meddlesome" governmental bodies, insurance companies, and hospital administrators, but is also indispensable for to co-operate in a setting of shared medical care, for medical audit, and for educational purposes.

In Chapter 5 we demonstrated that the implementation of a process view in electronic medical-record systems is technically not a problem. On the functional level, however, a barrier has to be taken yet. Recent initiatives also involving the process view had problems with implementing a user-interface of this view. It can be questioned whether this is merely a functional problem of user-interface design or also a conceptual problem of misperception between users and developers of electronic medical-record systems. It should not be forgotten that maintaining and using a process view is not part of the daily routine of medical-record keeping. We proposed a solution for this conceptual barrier by preparing a detailed data model of the process view based on the conversation model of systematic medical practice. Further study is needed to the design of an appropriate user-interface of the process view based on this new concept.

If a proper user-interface has been designed and implemented, it may then be possible to put the following questions on the research agenda. First, how can we measure the added value of the process view for direct medical practice? And second, what can be said about the optimal granularity for the presentation of medical narratives when also the process view is available? Different from the source view and problem view, which are only meant to facilitate the retrieval of clinical information, the process view is information in itself. While a lack of precision in source view or problem view can be compensated by reading the content of a paragraph, a less precise process view definitely results in a less accurate reconstruction of the process of medical practice. Further study is needed to determine the added value of the process view in relation to the granularity of medical narratives.

\subsubsection{Towards a conversation-based medical care?}

The next century may be the era of shared medical care. Even the patient will increasingly be involved in this process of sharing responsibilities. At present, etroneous communication is an increasing cause of medical failures. In shared medical care, the "clinical dialogue" should be more than just the communication of medical data between health-care professionals; also opinions, plans, and decisions need to be communicated. Hence, we welcome Frisse's idea of replacing medical data by conversations as the central metaphor of medical-information processing. ${ }^{2}$ Perhaps, the next century will be, above all, the era of conversation-based medical care.

\subsection{References}

1. Dick $\mathbb{R S}$, Stein EB. The computer-based patient record - an essential technology for health care. Washington DC: National Academy Press, 1991.

2. Frisse ME, Schnase JL, Metcalfe ES. Modells for patient records. Acad Med 1994; $69: 546-50$ 



\section{Samenvatting}

Bij elke medische handelingen registreert, raadpleegt of analyseert men medische gegevens. De belangrijkste opslagplaats van patiëntengebonden medische gegevens is het medisch dossier. Het primaire doel van het medisch dossier is het verschaffen van informatie voor directe zorgactiviteiten. In het verleden was het dossier voornamelijk een geheugensteun voor de individuele medicus, maar tegenwoordig geldt het dossier meer en meer als communicatiemiddel tussen de diverse zorgverleners rondom de patiënt.

\subsection{De mening van gebruikers over het papieren medisch dossier}

In de medische informatica gaat men er in het algemeen van uit, dat het papieren dossier zijn langste tijd heeft gehad en is men naarstig op zoek naar manieren om de ontwikkeling en verspreiding van electronische medische dossiers te bevorderen. Als men het dossier ziet als management-informatiesysteem, als bron voor wetenschappelijk onderzoek of voor andere secundaire doeleinden, kan men inderdaad constateren dat een electronisch medisch dossier (EMD) vele voordelen biedt. Ten aanzien van de primaire functie als raadpleegmiddel in de dagelijkse zorgpraktijk echter, blijken praktizerend artsen het oude vertrouwde papieren dossier nog steeds in hoge mate te waarderen. In Hoofdstuk 2 van dit proefschrift wordt een evaluatieve enquête beschreven, gehouden onder 85 ziekenhuisartsen van verschillende specialismen. Hierbij vonden wij, dat men met name het flexibele karakter van het papieren dossier op prijs stelde. Men wordt bijvoorbeeld niet beperkt in de keuze wat men wil registreren, in welke mate van detail en in welke woorden. Verder vond men de inhoud van het papieren dossier betrouwbaar genoeg om mee te werken. Het onderzoek bracht echter ook enkele tekortkomingen van het papieren dossier aan het licht. Zo vond men het raadplegen van het papieren dossier te langzaam en de registratie van beslissingen, plannen en opdrachten niet volledig genoeg. Ook de veiligheid en privacy-gevoeligheid van het papieren dossier lieten te wensen over. Uit dit onderzoek trokken wij de conclusie dlat, als men de invoering van een EMD overweegt, het aanbeveling verdient vooraf de mening van de gebruikers over bestaande praktijk van medische verslaglegging te peilen. Hierbij dient men niet alleen rekening te houden met eventuele wensen ter verbetering, maar ook met de aspecten van de bestaande praktijk waarover men tevreden is en die men wil behouden.

\subsection{Problemen met het automatiseren van "medical narratives"}

De tevredenheid onder medici over de papieren medische verslaglegging is niet het enige dat de ontwikkeling en verspreiding van EMD's in de weg staat. Uit 
literatuuronderzoek - beschreven in Hoofdstuk 3 - wordt duidelijk, dat veel belemmeringen het gevolg zijn van de moeite die men heeft met het automatiseren van de verslaglegging van anamnese, lichamelijk onderzoek en decursus. Deze gedeelten zijn verhalend van vorm, vandaar hun benaming medical narratives. Het onderzoek op dit gebied spitst zich toe op drie probleemgebieden: (1) Hoe kan de eenduidigheid van gegevens (met behulp van standaard terminologieèn) worden verhoogd? (2) Hoe kan de invoer van gegevens worden verbeterd? (3) Hoe kan het raadplegen van informatie worden vergemakkelijkt?

Het raadplegen van informatie uit de tekstuele gedeelten van het medisch dossier is op te splitsen in twee fasen. Eerst moet de gebruiker de paragraaf localiseren waarin hij de relevante informatie denkt te kunnen vinden. Deze fase kan worden ondersteund door de tekstuele gedeelten van een zoekstructum te voorzien, d.w.z. onder te verdelen op een dusdanige wijze dat de gebruiker gemakkelijk de relevante paragraaf kan vinden. Vervolgens moet de gebruiker de inhoud van de geselecteerde paragraaf doorzoeken om de informatie die hij zoekt te kunnen vinden. De inhoud van een paragraaf wordt in de meeste EMD's gepresenteerd als vrije tekst.

\subsection{Een zoekstructur met meerdere perspectieven}

De traditionele indeling van het medisch dossier is bron-georiënteerd, d.w.z. dat gegevens van gelijke herkomst en type bijeen staan. Om flexibeler te kunnen zoeken naar informatie wordt het traditionele dossier veelal aangevuld met andere indelingsprincipes: het indelen van medische gegevens naar probleem en het presenteren van medische gegevens als functie van de tijd. Van een dergelijke presentatie, ook wel flowsheets genoend, wordt veelvuldig gebruik gemaakt in bestaande EMD's - zowel in de bron-georiënteerde als in de probleem-georiënteerde indeling - zij het alleen voor niet-tekstuele gegevens.

In Hoofdstuk 4 hebben we drie alom bekende dossiertypen bestudeerd (de brongeoriênteerde, probleem-georiënteerde en tijd-georiënteerde) en hebben hieruit een viertal indelingsprincipes kunnen afleiden. Elk van deze principes is te beschouwen als een perspectief op medische gegevens.

- Een tijd-georienteerd perspectief: een ordening van gegevens op basis van registratietijdstip

- Een bron-georiënteerd perspectief: een hiërarchische indeling van gegevens naar herkomst en type

- Een probleem-georiënteerd perspectief, een selectie van gegevenstypen relevant voor een bepaald medisch probleem

- Een proces-georiënteerd perspectief: een selectie van gegevens naar de rol die zij hebben gespeeld bij de totstandkoming van medische zorg, bijvoorbeeld als indicatie of motivatie van een beslissing.

Vanuit een technisch oogpunt kunnen alle genoemde perspectieven worden gecombineerd in een en herzelfide gegevensmodel. Zodoende is een zoekstructuur denkbaar met vier invalshoeken. Vanuit een functioneel oogpunt echter levert de implementatie van het proces-georiënteerde perspectief de nodige problemen op. Deze 
problemen zijn het gevolg van een combinatie van factoren, waaronder de beperkingen van de huidige grafische gebruikersinterfaces en het ontbreken bij artsen van arvaring met systematische medische verslaglegging.

In Hoofdstuk 5 wordt betoogd, dat ook vanuit theoretisch oogpunt het procesgeoriënteerde perspectief - althans de meest gebruikelijke versie: Weed's SOAPstructuur - enkele beperkingen heeft. Dit geldt in het bijzonder wanneer men het dossier gebruikt in de setting van shared medical care. In de SOAP-structur is bijvoorbeeld niet nader uitgewerkt hoe een medisch oordeel tot stand komt. Ook komt in de SOAP-structuur niet tot uiting welke rol communicatie tussen zorgverleners speelt in het zorgproces. Een mogelijke oplossing voor deze problemen zou de introductie van een conversatiemodel van systematisch medisch handelen kunnen zijn. In dit model wordt het redeneren van de arts opgevat als een proces bestaande uit vier lagen die onderling zijn verbonden door middel van conversaties. De beschreven niveaus zijn: (1) het oplossen van patiëntenproblemen, (2) het oplossen van medische problemen, (3) het toetsen van hypothesen en (4) het uitvoerend medisch handelen. Het typerende van het conversatiemodel is, dat geen onderscheid wordt gemaakt tussen communicatie tussen verschillende personen en situaties waarin een persoon in gesprek is met zichzelf. Hierdoor is het model zowel toepasbaar op het zorgproces van de individuele arts als op shared medical care. Hoewel het conversatiemodel slechts een eerste poging tot een theoretische onderbouwing van shared medical care is, geloven we dat dit model bij uitstek geschikt is als referentiekader: niet alleen voor medische verslaglegging, maar ook voor bijvoorbeeld medical audit, medisch onderwijs en het indelen van besluitvormingondersteunende systemen naar "aangrijpingspunt" in het denkproces.

\subsection{Een electronisch medisch dossier met meerdere perspectieven}

In Hoofdstuk 6 van dit proefschrift wordt een zelf ontwikkeld EMD beschreven dat is toegespitst op de ondersteuning van het raadplegen van medical narratives. Dit systeem, dat een simpele, uniforme gebruikersinterface heef, is voorzien van een zoekstructuur bestaande uit bron-georiënteerde en probleem-georiënteerde flowsheets. Dit zijn tweedimensionale overzichten van gegevens met op de vertikale as een indeling van gegevenstypen (paragrafen) naar bron of probleem en op de horizontale as een tijdbalk. Als de gebruiker een deel van de flowsheet selecteert, krijgt hij toegang tot de inhoud van de betreffende paragrafen in het betreffende tijdsbestek. In het hier beschreven EMD bestaat de inhoud van anamnese, lichamelijk onderzoek en decursus uit vrije tekst. We hebben het systeem uitgebreid met een research module die een proefpersoon automatisch zoekinstructies geeft en daarbij elke aktie van de proefpersoon registreert.

\subsection{De optimale korrelgrootte van de zoekstructuur}

De meeste medische gegevens in een EMD worden gepresenteerd als afzonderlijke eenheden. Gegevens uit anamnese, lichamelijk onderzoek en decursus daarentegen 
worden veelal aaneengeregen tot een min of meer vloeiend betoog. Wel kan men deze medical narratives opdelen in paragrafen. Hoe gedetailleerder deze opdeling is (d.w.z. hoe kleiner de korrelgrootte), des te specifieker is de inhoud van elke paragraaf. Hierdoor kan men medical narratives - vanuit de verschillende invalshoeken selectiever raadplegen. Uit literatuurstudie blijkt, dat men er in bestaande EMD's uiteenlopende korrelgroottes op na houdt. Zo kan de decursus al dan niet zijn onderverdeeld in probleem-gerelateerde secties en kunnen anamnese en lichamelijk onderzoek bestaan wit één geheel, zijn opgesplitst in tractus c.q. lichaamsregio's, of zijn opgedeeld in afzonderlijke vragen c.q. observaties.

In Hoofdstuk 7 wordt beschreven, hoe we met het door ons ontwikkelde EMD drie verschillende dossierindelingen hebben bestudeerd, teneinde de optimale korrelgrootte vast te stellen voor een EMD met een zoekstructuur bestaande uit bron-georiënteerde en probleem-georiënteerde flowsheets. De proces-georiënteerde invalshoek hebben we vooralsnog buiten beschouwing gelaten. Voor dit doel hebben we drie dikke klinische casus uit de interne geneeskunde in de computer ingevoerd, en wel in alle drie de dossierindelingen. Vervolgens hebben we 36 medici (specialisten en artsassistenten) gevraagd over elk van deze casus enkele vragen te beantwoorden, aan de hand van de informatie die te vinden was in de tekstuele gedeelten van deze dossiers.

Men bleek een probleem-georiënteercle decursus sneller te kunnen raadplegen dan een ongedeelde decursus. Verder kon men informatie uit anamnese en lichamelijk onderzoek bij een opdeling in orgaansystemen (tractus c.q. lichaamsregio's) sneller vinden dan bij een opdeling in afzonderlijke vragen en observaties. Bij ongedeelde anamnese en lichamelijk onderzoek lag de gemiddelde zoeksnelheid tussen beide andere korrelgroottes in, echter zonder significant van beide andere te verschillen. We konden concluderen, dat een indeling van medical narratives in paragrafen het raadplegen bevordert - althans voor dikke dossiers - maar dat de meest gedetailleerde onderverdeling niet de beste behoeft te zijn. 


\section{Curriculum Vitae}

Huibert Tange werd geboren in 1958 te Dordrecht. Zijn lagere-schoolperiode bracht hij door in Middelburg en Krimpen aan den IJssel. Hij doorliep het Erasmiaans Gymnasium te Rotterdam en het Stedelijk Gymnasium te Arnhem, alwaar hij in 1977 eindexamen deed. Vervolgens begon hij de studie Geneeskunde te Nijmegen, welke hij in 1984 afsloot met het artsexamen. Tijdens zijn militaire dienst was hij als arts een half jaar werkzaam bij de UNIFIL-troepen in Libanon, waarna hij de rest van zijn diensttijd sportgeneeskundige ervaring opdeed op het Sport Medisch Centrum van de Koninklijke Landmacht te Utrecht. Deze ervaring heeft hij nadien nog in de praktịjk gebracht als keuringsarts op het Sportmedisch-Adviescentrum (SMA) te Sittard en in de medische begeleiding van de Nijmeegse Vierdaagse.

Vanaf 1986 is hij werkzaam als universitair docent bij de vakgroep Medische Informatica aan de Universiteit Maastricht. Zijn taak ligt deels in het verzorgen van onderwijs en deels in het verrichten onderzoek. Naast zijn reguliere werkzaamheden is hij onder meer betrokken geweest bij de opzet van post-academisch onderwijs en bij een Europees onderzoek naar de ontwikkeling van een electronisch medisch dossier (IRHIS) in het kader van AIM (Advanced Informatics in Medicine). Sinds 1991 heeft hij gewerkt aan zijn promotie-onderzoek waarvan dit proefschrift het resultaat is. De belangrijkste publicaties uit zijn promotie-onderzoek tot nu toe zijn hieronder weergegeven.

\subsection{Belangrijkste publicaties uit dit promotie-onderzoek}

1. Tange HJ, Donkers HHLM, Hasman A. Structuring Data in Medical Records, Proc 5th Int Conf on System Science in Health Care, Prague, 1992.

2. Tange HJ. Het medisch dossier in een ander perspectief : terug natar de zuivere betekenis van status praesens en decursus. Tijdschr Med Inf 1993; 22:30-33.

3. Tange $\mathrm{HJ}_{3}$ Smeets RPAM. Information exchange between physicians and nurses. Comp Meth Prog Biomed. Vol. 43, 1994:261-167.

4. Tange HJ, Vissers M. Hasman A, Donkers HHLM, van der Linden CJ. Computer supponted patient management. Techn Health Care. Vol. 1, 1994:253-264.

5. Tange HJ. The paper-based medical record: Is it really so bad? Comp Meth Prog Biomed 1995; 48:127-131.

6. Tange HJ, Dietz JLG, Vries Robbé PFd. A communication-oriented approach to systematic medical care: The problem-oriented medical record in a new perspective, Proceedings Amice'95, Amsterdam, 1995.

7. Tange HJ. How to approach the structuring of the medical record?: Towards a model for flexible access to free text medical data. Int J Biomed Comput 1996; 42:27-34.

8. Tange HJ, Schouten HC, Kester A, Hasman A. Het medisch dossier als raadpleegmiddel - Eem vergelijkend onderzoek naar verschillende structuren van "medical narratives", Proc Medische Informatica Congres '96, Brussel, 1996. 